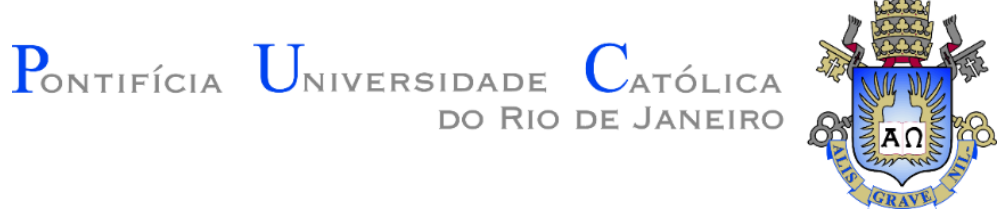

Giovanny Alberto Meneses Arboleda

\title{
AN EFFECTIVE COMPATIBILITY SCHEME IN MULTISCALE TOPOLOGY OPTIMIZATION OF STRUCTURES
}

Tese de Doutorado

Thesis presented to the Programa de Pós-graduação em Engenharia Mecânica of PUC-Rio, in partial fulfillment of the requirements for the degree of Doutor em Engenharia Mecânica.

Advisor: Prof. Anderson Pereira 


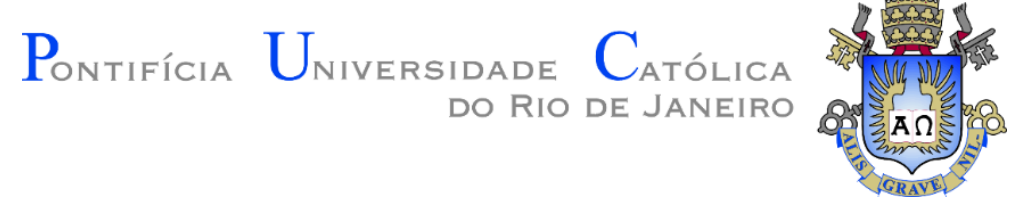

Giovanny Alberto Meneses Arboleda

\section{AN EFFECTIVE COMPATIBILITY SCHEME IN MULTISCALE TOPOLOGY OPTIMIZATION OF \\ STRUCTURES}

Thesis presented to the Programa de Pós-graduação em Engenharia Mecânica of PUC-Rio, in partial fulfillment of the requirements for the degree of Doutor em Engenharia Mecânica. Approved by the Examination Committee.

Prof. Anderson Pereira Advisor

Departamento de Engenharia Mecânica - PUC-Rio

Prof. Ivan Fábio Mota de Menezes Departamento de Engenharia Mecânica - PUC-Rio

Prof. Arthur Martins Barbosa Braga Departamento de Engenharia Mecânica - PUC-Rio

Prof. Rodrigo Bird Burgos

Departamento de estruturas e fundações - UERJ

Prof. Francisco José da Cunha Pires Soeiro Departamento de Engenharia Mecânica - UERJ 
All rights reserved.

\section{Giovanny Alberto Meneses Arboleda}

The author received his bachelor's degree in Mechatronics Engineering in 2012 from the Universidad Santo Tomas de Aquino Bucaramanga, Colombia. Obtained his master's degree in Mechanical Engineering with emphasis on Applied Mechanics from the Pontifícia Universidade Católica do Rio de Janeiro (PUC-Rio) in 2015.

Bibliographic data

Meneses Arboleda, Giovanny Alberto

An effective compatibility scheme in multiscale topology optimization of structures / Giovanny Alberto Meneses Arboleda ; advisor: Anderson Pereira. - 2021.

128 f. : il. color. ; $29,7 \mathrm{~cm}$

Tese (doutorado)-Pontifícia Universidade Católica do Rio de Janeiro, Departamento de Engenharia Mecânica, 2021.

Inclui bibliografia

1. Engenharia Mecânica - Teses. 2. Otimização topológica. 3. Manufatura aditiva. 4. Otimização topológica multiescala. 5. Otimização de microestruturas treliçadas com densidade variável. 6 . Material anisotrópico poroso com penalização. I. Pereira, Anderson. II. Pontifícia Universidade Católica do Rio de Janeiro. Departamento de Engenharia Mecânica. III. Título.

CDD: 621 
To my mom and dad, Melida y Reinaldo, for all their love and support. 


\section{Acknowledgments}

My appreciation to my advisor, Prof. Anderson Pereira, for the support and advice throughout the development of my dissertation, to Prof. Ivan F. Mota de Menezes, for believing in me and his constant words of encouragement.

I would like to thanks my family who through their love gave encouraged me to give my best. I thank Laura E. Cué, for her love and affection; with her help, everything was easier. To Guillermo Domech for his invaluable help.

I am grateful to all those people who indirectly helped me to complete this thesis.

This study was financed in part by the Coordenação de Aperfeiçoamento de Pessoal de Nível Superior - Brasil (CAPES) -Finance Code 001, and Conselho Nacional de Desenvolvimento Científico e Tecnológico (CNPq). 


\section{Abstract}

Meneses, Giovanny Alberto Arboleda; Anderson Pereira (Advisor). An Effective Compatibility Scheme in Multiscale Topology Optimization of Structures. Rio de Janeiro, 20210. 128p. Doctoral thesis - Mechanical Engineering Department, Pontifical Catholic University of Rio de Janeiro.

Recent advances in additive manufacturing techniques have increased their flexibility in making complex parts on a smaller scale. In this context, the design of porous microstructures has been standing out in the scientific community due to the ability to optimize the cell topology to meet the design requirements. However, there are several challenges that inhibit the fabrication of optimized parts obtained by the multi-scale topology optimization method, such as the connectivity of microstructures. The multiscale topological optimization consists of the optimization of both the macro-scale, global structure, and the micro-scale, microstructure of the material. The main objective of this work is to develop an effective scheme to guarantee compatibility in the transition between the different material microstructures obtained in multiscale optimization. The multiscale methodologies for simultaneous topological optimization of both scales and the homogenization procedures are described. The main numerical and computational aspects of these methods are presented, as well as representative examples to illustrate the capabilities of the proposed scheme.

\section{Keywords:}

Topology Optimization; Additive Manufacturing; Multi-Scale Topology Optimization; Variable-Density Lattice Structure Optimization; Porous Anisotropic Material with Penalization. 


\section{Resumo}

Meneses, Giovanny Alberto Arboleda; Anderson Pereira. Um Esquema Eficaz de Compatibilidade na Otimização Topológica Multiescala de Estruturas. Rio de Janeiro, 2021. 128p. Tese de Doutorado - Departamento de Engenharia Mecânica, Pontifícia Universidade Católica do Rio de Janeiro.

Os recentes avanços das técnicas de manufatura aditiva vêm ampliando a sua flexibilidade em fabricar peças complexas em escala cada vez menores. Neste contexto, o projeto de microestruturas porosas vem se destacando na comunidade científica devido a capacidade de se otimizar a topologia da célula para atender aos requisitos de projeto. No entanto, existem vários desafios que dificultam a fabricação de peças obtidas pelo método de otimização topológica multiescala, dentre eles, a conectividade das microestruturas. A otimização topológica multiescala consiste na otimização tanto da macroescala, estrutura global, quanto da microescala, microestrutura do material. O objetivo principal deste trabalho é desenvolver um esquema eficaz para garantir a transição entre as diferentes microestruturas de material obtidas na otimização multiescala. As metodologias multiescala de otimização topológica simultânea de ambas as escalas e os procedimentos de homogeneização são descritos. Apresentam-se os principais aspectos numéricos e computacionais destes métodos, assim como exemplos ilustrativos.

\section{Palavras-Chave:}

Otimização Topológica; Manufatura Aditiva; Otimização Topológica Multiescala; Otimização de Microestruturas Treliçadas com Densidade Variável; Material Anisotrópico Poroso com Penalização. 


\section{Table of Contents}

1 Introduction 23

$\begin{array}{lll}1.1 & \text { Hierarchical Materials } & 23\end{array}$

1.2 Structural Optimization 25

$\begin{array}{lll}1.3 & \text { Problem Formulation } & 27\end{array}$

$\begin{array}{lll}1.4 & \text { Objectives } & 29\end{array}$

1.5 Thesis Outline 30

2 Literature Review on Multiscale Topology Optimization 32

2.1 Design of Porous Solid 32

2.1.1 Single-Scale Method 32

2.1.2 Multiscale Method 35

2.1.2.1 Single Material or Single Cell 36

$\begin{array}{ll}\text { 2.1.2.2 Multi-material or Multi-region } & 37\end{array}$

2.2 Additive Manufacturing (AM) 41

$\begin{array}{lll}2.2 .1 & \text { Stereolithography } & 41\end{array}$

2.2.2 Selective Laser Sintering 42

2.2.3 Fused Deposition Modelling 43

3 Multiscale Topology Optimization 45

3.1 Topology Optimization (TO) 45

$\begin{array}{lll}3.1 .1 & \text { Sensitivity Analysis } & 47\end{array}$

3.1.2 Optimality Criteria Method 48 
$\begin{array}{lll}3.1 .3 & \text { Filtering } & 49\end{array}$

3.2 Solid Isotropic Material with Penalization (SIMP) 50

3.3 Porous Anisotropic Material with Penalization 51

3.3.1 Sensitivity Analysis $\quad 54$

3.3.1.1 Macroscale $\quad 55$

3.3.1.2 Microscale $\quad 56$

3.3.2 PAMP Multi-region $\quad 56$

$\begin{array}{lll}3.4 & \text { Homogenization } & 60\end{array}$

3.5 Connections Methods 63

3.5.1 Kinematical Connective Constraint Method (KC) 64

$\begin{array}{lll}\text { 3.5.2 Pseudo Load } & 64\end{array}$

4 Transitional Microstructure for Smooth Connections Between

Adjacent Regions (TrMIC) 66

$\begin{array}{lll}4.1 & \text { Motivation } & 66\end{array}$

$\begin{array}{lll}4.2 & \text { Proposed Method } & 68\end{array}$

$\begin{array}{lll}4.3 & \text { Final Considerations and Discussion } & 71\end{array}$

5 Numerical Examples $\quad 72$

5.1 Evaluating the implemented codes 72

$\begin{array}{lll}5.1 .1 & \text { Homogenization } & 72\end{array}$

5.1.2 Single Region MBB-beam Problem 73

5.1.3 Single Region L-shape Problem 75

$\begin{array}{lll}5.2 & \text { Experiments for PAMP } & 78\end{array}$

5.2.1 Experiments for the MBB-beam Problem 78

5.2.2 Experiments for the L-shape Problem 84

$\begin{array}{lll}\text { 5.3 3D Printed Samples } & 90\end{array}$

$\begin{array}{lll}5.4 & \text { ANSYS Simulation } & 94\end{array}$ 
$\begin{array}{lll}\text { 5.4.1 Classic TO } & 94\end{array}$

$\begin{array}{lll}\text { 5.4.2 Variable-density } & 98\end{array}$

6 Conclusions and Future Works 102

6.1 Future Work 103

$7 \begin{array}{ll}705 \\ \end{array}$ References 105

$\begin{array}{lr}\text { Appendix } & 128\end{array}$ 


\section{Figures}

Figure 1-1 - Hierarchical structure of bamboo [1].

Figure 1-2 - a) 2D Honneycomb b) Open-Cel foams c) Closed-Cell foams [3].

Figure 1-3 - Structural optimization approaches [10]. On the left side are the initial problems and on the right side are the optimal solution. (a) Sizing optimization, (b) Shape optimization, and (c) topology optimization.

Figure 1-4 - Illustration of multiscale TO of structure and materials [28]. 26 Figure 1-5 - Disconnected microstructures using Level Set method [58]. 28 Figure 1-6 - Challenges in existing methods for compatibility microstructure: a) imperfect connection using implicit control method with genetic algorithms [63]. b) connectivity problem using threedimensional shape [61].

Figure 2-1 - a) Foam infill [66]. b) Grid pattern infill [67, 68]. c) Honeycomb-cells infill [69]. d) Rhombic infill [40].

Figure 2-2 - a) MBB-beam Lattice structures [70]. b) hybrid Variabledensity with lattice [78]. c) Cantilever beam with nonuniform lattice [79]. 34 Figure 2-3 - a) Porous bone-like solution [80], b-d) Microstructures to Control Elasticity [59].

Figure 2-4 - A structure composed of a porous anisotropic material [44].36

Figure 2-5 - a) TO with BESO [45]. b) TO using (LSM) [48]. c) TO using MIST [49].

Figure 2-6 - a) TO with non-linear analysis [28].b) TO decomposed with LSM [51] c) TO with multi-patch microstructures [52]. 
Figure 2-7 - a) cells by density distribution [89]. b) PAMP and non-uniform microstructures [90]. c) TO with layer-wise sectorization [60].

Figure 2-8 - a) hierarchical optimization [53]. b) MTOP [54].

Figure 2-9 - Microstructure self-compatibly issues, adapted from $[28,51]$.

Figure 2-10 - a) Shape metamorphosis [61]. b) BESO FGM [63]. c) Compound cells [64].

Figure 2-11 - a) Microbull made by two-photon microstereolithography.

b) micro-oscillator system. Scale bar $2 \mu \mathrm{m}$ [105].

Figure 2-12 - Two-component liquid-phase sintering of Cu-Sn. Line width $=470 \mu m[108]$.

Figure 2-13 - FDM bone-like porous infill [80].

Figure 3-1 - Illustration of Domain and boundary conditions.

Figure 3-2 - Flowchart of the topology optimization process.

Figure 3-3 - Density $(\rho)$ against penalized density $(\rho p)$ for different penalization factors $p$.

Figure 3-4 - A macrostructure composed of a porous anisotropic material.

Figure 3-5 - Flowchart of the Porous Anisotropic Material with Penalization process.

Figure 3-6 - Macrostructure optimized with DMO and composed of three different materials.

Figure 3-7 - An arbitrary Macrostructure composed of multiple porous regions.

Figure 3-8 - Periodic Boundary Conditions (PBC), a) Base cell. b) Deformation induced by unit initial strain in the vertical direction. $\mathrm{C}$ ) 
Deformation induced by unit initial strain in the horizontal direction. d)

Deformation induced by unit initial shear strain.

Figure 3-9 - Rectangular cell model in 2D adapted from [32].

Figure 3-10 - Kinematical connector adapted from [62].

Figure 3-11 - Pseudo Load adapted from [62].

Figure 4-1 - Topologically optimized solid with two different types microstructures with discontinuities.

Figure 4-2 - Muscle, tendons, and bone [178].

Figure 4-3 - Two cell (1 and 2) bonded by the TrMIC (3).

Figure 4-4 - a) TrMIC under non-PCB. b) Case 1: load on the right (fixed-left) and c) Case 2: load on the left (fixed right).

Figure 4-5 - Two-step PL-based process. a) First iterations. b) Intermediate iterations. c) Final topology.

Figure 5-1 - Comparison between homogenized tensors.

Figure 5-2 - MBB-beam example (macro and micro design) adapted from [44].

Figure 5-3 - MBB-beam using multiscale composite [84].

Figure 5-4 - Results of PAMP MBB-beam 1 cell.

Figure 5-5 - L-shape problem 1.

Figure 5-6 - L-shape using multiscale composite [84]. 76

Figure 5-7 - Results and comparison of L-shape problem. 77

Figure 5-8 - Decomposition of the L-shape beam [44]. 78

Figure 5-9 - Half MBB-beam solution using PAMP with 1 cell. $\quad 79$

Figure 5-10 - Half MBB-beam solution using PAMP with 3 cells, a) macrostructure, b) microstructure, and homogenized stiffness tensor. 
The orange ellipse denotes the regions where did not exists a connection between cells or the connection is poor.

Figure 5-11 - Half MBB-beam solution using PAMP TO with 5 cells, a) macrostructure, b) microstructure, and homogenized stiffness tensor. The red ellipse denotes the regions where did not exists a connection between cells or the connection is poor.

Figure 5-12 - Half MBB-beam solution using PAMP TO with 5 cells and TrMIC, a) macrostructure, b) microstructure, and homogenized stiffness tensor.

Figure 5-13 - MBB-beam solution from [55] 83

Figure 5-14 - L-shape problem 2.

Figure 5-15 - L-shape solution using PAMP. a) macrostructure, b) microstructure, c) stiffness matrix.

Figure 5-16 - L-shape solution using PAMP with 3 cells, a) macrostructure, b) microstructure, and c) homogenized stiffness tensor. The orange circle denotes the regions where did not exists a connection between cells or the connection is poor.

Figure 5-17 - L-shape solution using PAMP with 5 cells. a) macrostructure, b) microstructure and c) homogenized stiffness tensor. The red circles denotes the regions where did not exists a connection between cells or the connection is poor.

Figure 5-18 - L-shape solution using PAMP with 5 cells and TrMIC. a) macrostructure, b) microstructure and c) homogenized stiffness tensor. 88 Figure 5-19 - L-shape with $\mathrm{Cl}$ solution a) macrostructure, b) from [58]. 89 Figure 5-20 - MBB-beam solution using Classic Topology optimization. 90 Figure 5-21 - MBB-beam solution using Variable-Density Topology Optimization.

Figure 5-22 - MBB-beam solution using a) PAMP multi-region TO and the 3D printed solid. 
Figure 5-23 - MBB-beam disposition for the three-point flexural test. a) Classic TO, b) Variable-density.

Figure 5-24 - Three points flexural test for classic and Variable-density 3D printed samples.

Figure 5-25 - Simulation of a 3D printed solid from [188].

Figure 5-26 - ANSYS force and supports and force for Classic TO analysis.

Figure 5-27 - ANSYS boundary conditions for Classic TO analysis. $\quad 94$

Figure 5-28 - ANSYS Mesh refinement for TO classic. 95

Figure 5-29 - ANSYS Von-Mises stress for Classic TO. 96

Figure 5-30 - ANSYS total strain for Classic TO. 96

Figure 5-31 - ANSYS total deformation for Classic TO. 97

Figure 5-32 - Comparison TO. 97

Figure 5-33 - ANSYS force and support for Variable-density TO. 98

Figure 5-34 - ANSYS boundary conditions for Variable-density TO. 98

Figure 5-35 - ANSYS Von-Mises stress for Variable-density. 99

Figure 5-36 - ANSYS equivalent total strain for Variable-density. $\quad 99$

Figure 5-37 - ANSYS total deformation for Variable-density. 100

Figure 5-38 - ANSYS mesh convergence analysis for Variable-density. 100

Figure 5-39 - Comparison Variable-Density.

Figure A-1 - Topology optimization. a) Half MBB-beam b) $p=1$ b) $\mathrm{p}=1-3$.

Figure A-2 - MBB-beam Variable-density. 


\section{Tables}

Table 5-1 - Comparison of half MBB-beam using PAMP. 83

Table 5-2 - Comparison L shape PAMP. $\quad 89$

Table 5-3 - Material Properties for ANSYS. 95

Table 5-4 - Comparison of 3D printed models, physical and ANSYS tests. 


\section{List of Symbols}

A $\quad$ : L-shape side.

$A_{M A} \quad$ : Macroscale area.

$A_{M I} \quad:$ Microscale area.

B : Strain/displacement matrix.

c : Compliance.

$d \quad$ : Number of Dimensions.

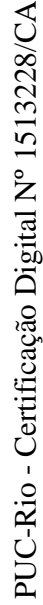

e : Element index.

E : Young's modulus.

$\mathbf{D}^{B} \quad$ : Base material elastic matrix.

$\mathbf{D}_{e} \quad$ : Elastic matrix from different materials.

$\mathbf{D}^{H} \quad$ : Homogenized elastic matrix.

$\quad \quad$ : Homogenized elastic matrix target.

$\mathbf{D}_{\varpi} \quad$ : Homogenized elastic matrix of candidate material.

$\mathbf{D}^{M A} \quad$ : Macroscale elastic matrix.

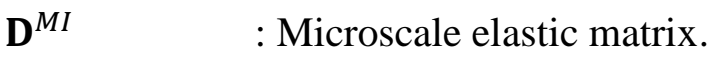

$\mathrm{E}_{0} \quad$ : Material Young's modulus.

$\mathbf{D}_{e} \quad$ : Element elastic matrix.

$\mathrm{E}_{\min } \quad:$ Minimum allowed stiffness.

F : Global force vector.

$k-/+\quad$ : Opposite parallel surfaces. 
$\mathbf{K}_{0} \quad$ : Element stiffness matrix with unitary Young's module.

K : Global stiffness matrix.

$N_{\varpi} \quad:$ Number of candidate materials.

$p \quad$ : Penalization factor for SIMP

$p_{\varpi} \quad:$ Penalization factor for discrete material optimization.

$p_{M A} \quad:$ Penalization factor at the macroscale

$p_{M I} \quad:$ Penalization factor at the microscale.

rmin : Minimum radius for the Optimization process.

$\operatorname{rmin}_{M A} \quad:$ Minimum radius for the optimization process at the macroscale.

$\operatorname{rmin}_{M I} \quad$ : Minimum radius for the optimization process at the microscale.

$\mathbf{u}^{*}$

: Periodic fluctuation.

$\mathbf{u}^{\mathbf{A}} \quad$ : Element displacement of the unit strain field.

$\mathbf{u}^{e} \quad$ : Displacement field.

$\mathbf{U}_{e} \quad:$ Vector of Local displacement.

$\mathbf{U} \quad$ : Vector of global displacement.

$V_{0} \quad$ : Volume domain.

$V_{(\rho)} \quad$ : Volume design.

$\bar{v} \quad$ : Macroscale volume fraction.

$\bar{v}_{M I} \quad:$ Microscale volume fraction.

$V_{f} \quad:$ Prescribed volume fraction.

$\mathbf{w} \quad$ : Weight factor of the candidate material.

$\mathbf{X} \quad$ : Set of all design variables.

$\boldsymbol{x}$

: Design variable at discrete material optimization.

Y

: Area or volume of the cell. 


\section{Greek letters}

$\alpha \quad$ : Penalization factor in discrete material optimization.

$\boldsymbol{\varepsilon}^{0} \quad$ : Unit test strain field.

$\boldsymbol{\varepsilon}^{A} \quad:$ Superimposed strain field.

$\boldsymbol{\varepsilon}^{*} \quad:$ Periodic strain field.

$\eta \quad$ : Weight factor in the Pseudo Load.

$\lambda \quad$ : Weight factor for L-shape problem.

$v \quad$ : Poisson's ratio.

๘ $\quad$ : Set of candidate materials.

$\rho \quad$ : Material density.

$\rho^{P A M} \quad:$ Relative density of the porous material.

$\boldsymbol{\rho}_{e} \quad$ : Design variable- Element densities.

$\rho_{M A} \quad:$ Macroscale density.

$\rho_{M I} \quad:$ Microscale density.

$\omega \quad$ : Set of material points that define the optimal shape.

$\Omega \quad$ : Set of material points that define the design domain. 


\section{List of Acronyms}

2D : Two-Dimensional Space.

3D : Three-Dimensional Space.

AM : Additive Manufacture.

ASTM : American Society for Testing and Materials.

BESO : Bi-directional Evolutionary Structural Optimization.

CI : Connectivity Index.

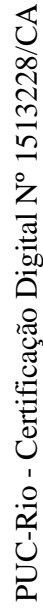

DLMS : : Direct Metal Laser Sintering.

DMO : Discrete Material Optimization.

EBHM : Energy-Base Homogenization Method.

FE $\quad$ : Finite Elements.

FE 2 : Multilevel Finite Element Method.

FEM : Finite Element Method.

FDM : Fused Deposition Modeling.

FFF $\quad$ : Fused Filament Fabrication.

FGM : Functionally Graded Material.

IGA : Isogeometric Analysis.

ITO : Isogeometric Topology Optimization.

KC : Kinematical connector.

KPI : Key Performance Indicator.

LA : Lattice Approach. 
LSM : Level Set Method.

MEMS : Microelectromechanical System.

MIST : Moving Iso-Surface Threshold.

MMA $\quad$ : Method of Moving Asymptotes.

MTOP : Multi-resolution Topology Optimization.

NURBS : Non-uniform rational B-spline.

OC : Optimality Criteria

PAMP : Porous Anisotropic Material with Penalization.

PBC : Periodic Boundary Conditions.

PL : Pseudo Load.

\begin{tabular}{|c|c|}
\hline SIMP & Isotropic Material with Penalization. \\
\hline SLA & : Stereolithography Apparatus. \\
\hline SLM & : Selective Laser Melting. \\
\hline SLS & : Selective Laser Sintering. \\
\hline TO & : Topology Optimization. \\
\hline TrMIC & : Transitional Microstructure. \\
\hline UF & : Unified Formulation. \\
\hline VSFL & : Velocity Field Level Set. \\
\hline
\end{tabular}


"It is curious how often you humans manage to obtain that which you do not want." Sr. Spock 


\section{Introduction}

\section{1}

\section{Hierarchical Materials}

In nature, several materials are able to combine stiff and soft components, such as nacre, bone, and silk; or stiff and porous elements like cancellous bone or wood. These combinations were designed for specific purposes. For example, in many of these materials, the soft phase works as a toughness mechanism in a fracture process. The hard phase, a highly mineralized material, minimizes wear and provides protection. Stiff-porous materials are characterized as lightweight and high stiffness but tend to be weak [1]. These materials use different structures or structural orientations to achieve different objectives. This kind of arrangement is called hierarchical structures. As defined by Lake in [2], hierarchical structures contain structural elements which themselves have structure and their hierarchical order may be defined as the number of levels of scale with recognized structure. The minimum order scale is zero (0) and can be viewed as continuous solid. Order one (1) can be seen either as a latticework of the continuous ribs or the ordered arrangement of atoms (or molecules) in a crystalline material. Figure 1-1 shows the hierarchical structure of bamboo. Bamboo is composed of cellulose fibers imbedded in a lignin-hemicellulose matrix shaped into hollow prismatic cells of varying wall thickness [1].

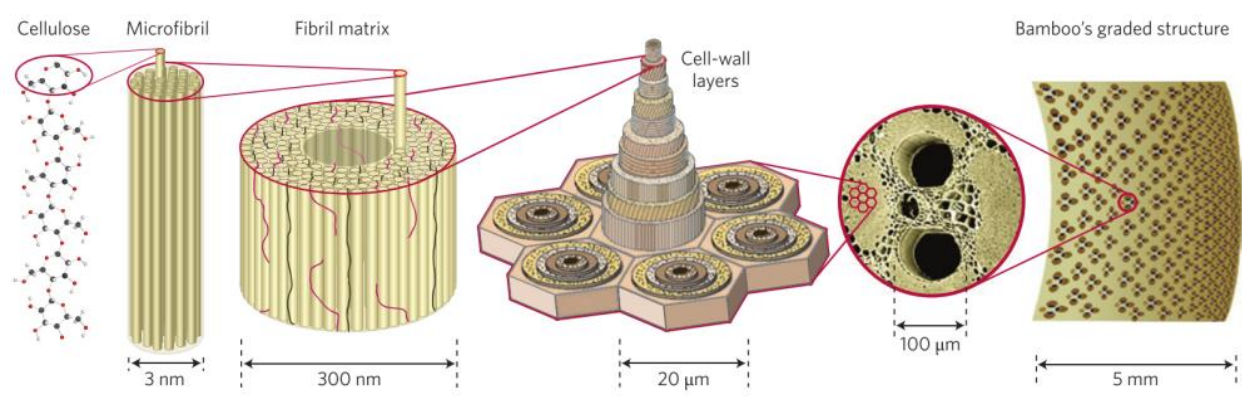

Figure 1-1 - Hierarchical structure of bamboo [1]. 
In this context, cellular solids play an important role. Cellular solids consist of an array of periodic structures called cells, which are composed of two phases: one is solid and the other one is an empty space or a fluid [2]. These cells are composed of small compartments that can be closed or open and can be grouped by periodic or stochastic processes in 2D or 3D structures. Examples of these structures are honeycombs, Figure 1-2, corks, sponges, and foams [3, 4], Figure 1-2. Cellular solid are not hierarchical but can be used to make hierarchical structures [2].

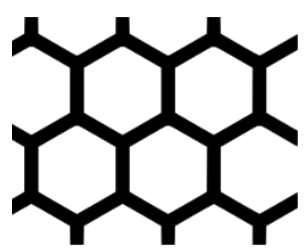

a)

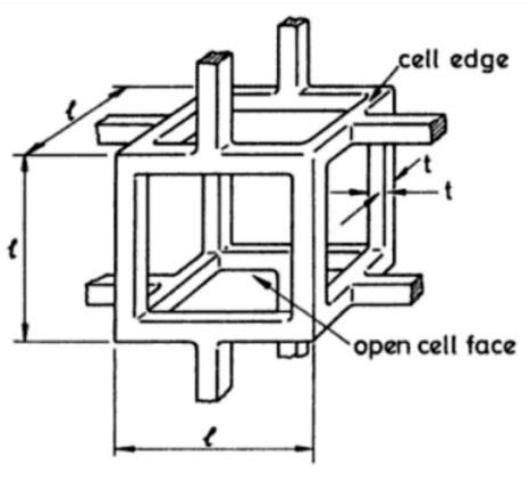

b)

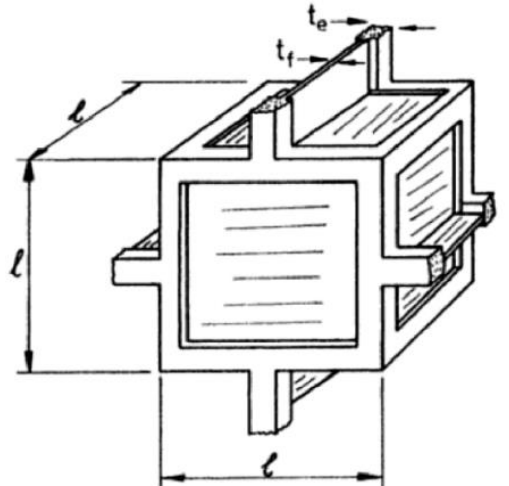

c)

Figure 1-2 - a) 2D Honneycomb b) Open-Cel foams c) Closed-Cell foams [3].

The cellular solids extend the range of physical, mechanical, and thermal properties, which creates applications that cannot easily be filled by dense solids [3]. Having low densities allows for lightweight and stiff components, in both static and dynamic applications. Some examples of these applications include sandwich panels and portable structures $[3,5]$. Having low thermal conductivity enables thermal insulation, which only can be beaten with expensive vacuum methods. Low stiffness enables cushion applications. With low strengths and large compressive strains, cellular solids are a good choice for energy-absorbing applications (that allows product packaging), Also enables other properties as tunable thermal expansion, buoyancy, among others [3, 6-9]. These applications are suitable for almost all industries. 


\section{2}

\section{Structural Optimization}

Structural optimization problems are related to the minimization or maximization of the structure response when is subject to some constraints, such as compliance, deflection, or peak stress. There are three different approaches to solve structural optimization problems: size, shape, and topology [10]. In a sizing problem, the objective is to find the optimal size for a given structure, for example, find the optimal thickness distribution of the area in a truss structure. In a shape optimization problem, the aim is to find the optimum shape of the domain, modifying its contour. Finally, the topology optimization problem implies determining the number of holes, the form, and the connectivity along with the domain. Figure 1-3 shows the three different approaches abovementioned.

(a)

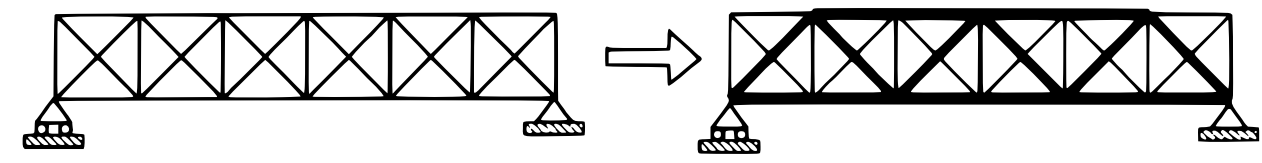

(b)

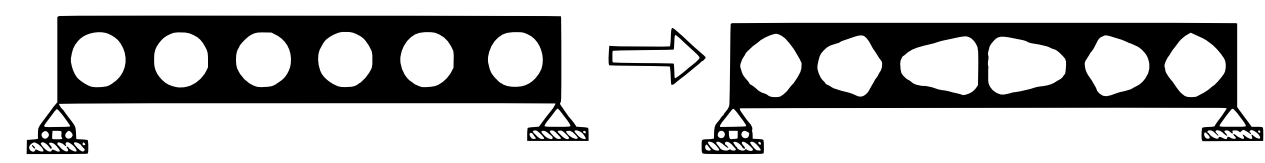

(c)

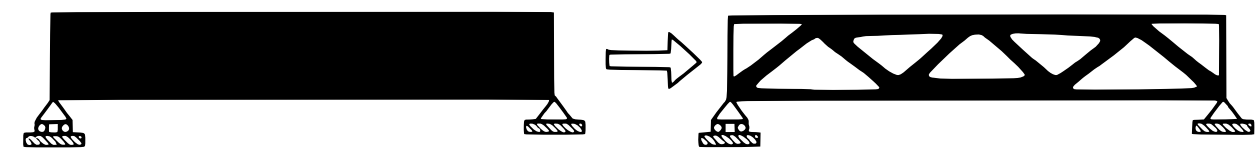

Figure 1-3 - Structural optimization approaches [10]. On the left side are the initial problems and on the right side are the optimal solution. (a) Sizing optimization, (b) Shape optimization, and (c) topology optimization.

Originally, Topology Optimization (TO) was conceived as a binary or discrete problem (0-1) and therefore can be considered poorly conditioned, leading to a lack of existence of solution [11]. Bendsøe et al. [12] relaxing the problem by assuming the microstructure (i.e. microscale) as a homogeneous material. Similar approaches follow [13-15]. Later, Bendsøe [16] proposed a more simplified density-based method also know as Solid Isotropic with Penalization (SIMP), which focused the TO research efforts on the optimization of single-scale structures (i.e. macroscale), generating a revolution in both the research $[17,18]$ and industry community [1922]. Thanks to the advance in the field of materials science, and the new facilities in the control of microstructure composition [23-25], the idea of concurrently 
designing both the macro and microscale came to the spotlight. News approaches were proposed suggesting materials tailored for the specific application, where every point in the structure should be designed to the specific local load conditions. The idea of tailored materials was presented by Ashby [26], suggesting the design of structural shapes in combination with material microstructures. In [2], Lakes discussed the use of hierarchical structures and the role of rapid prototyping in their manufacture. Bendsøe et al. [27] suggest that the optimal structure can be obtained by the free parametrization of the elasticity tensor.

The multiscale topology optimization determines the optimal spatial material layout distribution at the macroscopic scale and the optimal local use of the cellular material at the microscopic scale [28] (see Figure 1-4). To model the microstructure, the homogenization is commonly applied to link both structural an material scales $[13,29-32]$. In the homogenization is assumed that the microscopic length scale is much smaller than the macroscopic length scale, and the microstructure model can be considered as periodically ordered pattern. The key hypotheses of the homogenization are the separation of scales and the periodicity [32].

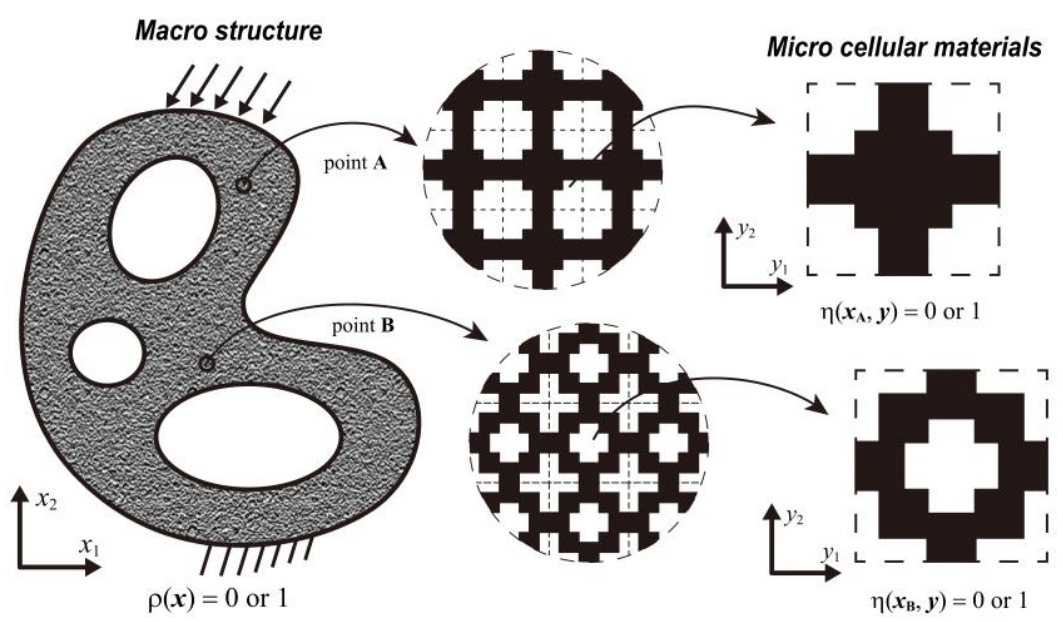

Figure 1-4 - Illustration of multiscale TO of structure and materials [28].

TO has been used in a wide range of applications such as aerospace, automotive, electromagnetic, electrochemical, electrothermal actuators, piezoelectric transducers, Microelectromechanical systems (MEMS), Stokes flows problems, acoustic devices, bio-mechanical simulations, etc [17]. However, due to the complexity of these forms, new manufacturing problems have emerged that cannot 
be solved with traditional manufacturing methods, but can be avoided with additive manufacture. Some of these problems include the limitations to construct complicated internal patterns, the use of multiples material in a single piece, fully functional assembled mechanisms, high customization, among other [33, 34].

The American Society for Testing and Materials (ASTM) [35] defines the Additive Manufacture (AM) technique as the process of joining materials to make objects from 3D model data, usually layer upon layer, as opposed to subtractive manufacturing methodologies. Allows the creation of very complex solids and high customization capacity with low material waste compared to traditional manufacturing processes. Also, it is characterized by having great compatibility with different materials. However, this manufacturing technique has drawbacks as well [36-42]. In particular, it is not structurally self-supporting during the manufacturing process $[39,43]$, and the combination of nanoscale precision and the fabrication of large-scale components turn out this technique a slow process. Nevertheless, the self-supporting restriction can be considered in the design process without sacrificing versatility when building complex solids. This technology, far from being the ideal way of fabricating hierarchical structures, for now, provides a powerful platform for isolating and testing specific design concepts [1].

\section{3 Problem Formulation}

TO can be used to design hierarchical structures with predefined properties, such as materials with negative Poisson's ratio, maximum bulk modulus and maximum shear modulus. To this aim, several frameworks have been proposed. Liu et al. [44] introduced the Porous Anisotropic Material with Penalization (PAMP) method, a concurrent scheme based on the SIMP method. Yan et al. [45] Perform the TO using genetic algorithms [46, 47], among others [48-50]. Therefore, it seems natural to exploit this capability to create a porous solid object with different cellular regions, where each region can have different properties. In this sense, some schemes have been proposed [28, 51-55]. Xia et al. [28] propose a FE2 nonlinear multiscale analysis using genetic algorithms. Sivapuram et al. [51] introduces a multiscale optimization using Level Set method [56] that allows any number of unique microstructures. Wang et al. [55] present a concurrent two-scale optimization 
framework combing the velocity level set method [57] and SIMP method. However, as geometric complexity increases, the connectivity of the microstructures becomes a key limitation. Figure 1-5 shows the macroscale (blue) and the microscale (red) illustrating the connectivity issues.
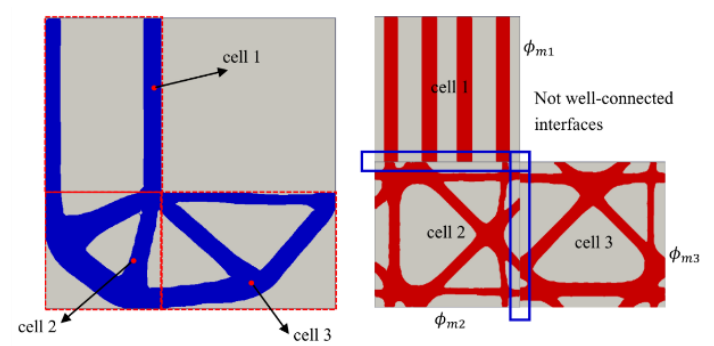

Figure 1-5 - Disconnected microstructures using Level Set method [58].

The irregularities in the interface create stress concentration points causing crack propagation or even internal penetration, which can lead to catastrophic failures on large scale. To overcome this problem, some approaches have been proposed [52, 55, 58-64]. Schumacher et al. [59] proposed interchanging different prescribed cells with similar properties to improve compatibility. Du et al. [58] suggest penalizing the cell's border area to enforce the connection. Zhang et al. [60] proposed dividing the body into layers to have a smooth transition; Wang et al. [61] use a three-dimensional shape to ensure partial connection in a 2D space. Zhou et al. [62] proposed three methods: 1) Pseudo Load (PL), 2) kinematical connective constraints (KC), and 3) the Unified formulation (UF) with nonlinear diffusion. Both, PL and KC methods employs a fixed area in the cell that functions as an interface. Despite the advances, these approaches cannot ensure well-connected transitions (see Figure 1-6) and the entire regions needs to be modified to achieve good connectivity. This involves restricting the design space of the solution to match connectivity, resulting in a decrease in stiffness [65]. 


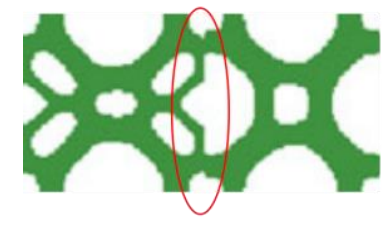

a)

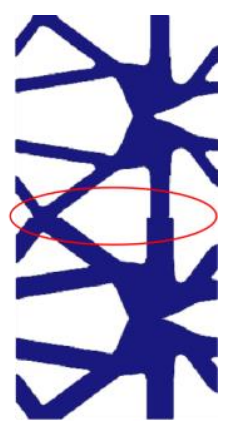

b)

Figure 1-6 - Challenges in existing methods for compatibility microstructure: a) imperfect connection using implicit control method with genetic algorithms [63]. b) connectivity problem using three-dimensional shape [61].

This work builds on that literature and proposes a novel compatibility scheme to ensure the connection between adjacent cells in a multicellular solid without substantially compromising stiffness. The scheme consists of a transition microstructure that bonds two different cellular groups using a non-periodic cell. The proposed approach uses the PAMP method, a density-based method, to perform the TO of the hierarchical structure, the homogenization process is used to link the scales and a two-step Pseudo Load-based method combined with KC to ensure the compatibility on the transition microstructure.

\section{4 \\ Objectives}

The main objective of this thesis is to propose a compatibility scheme to ensure the connectivity between cells in a hierarchical solid with different kinds of microstructures. In order to achieve this objective, the following specific objectives have been set:

- Define the mathematical formulation for the multiscale topology optimization approach and the compatibility scheme.

- To implement the code to design a porous material by means of a multiscale topology optimization method for single and multi-region. 
- To test the proposed scheme with different problems and compare it with the existing solutions to show the effectiveness.

\section{5}

\section{Thesis Outline}

Chapter 1: This chapter is the introduction to this research. Section 1.1 describes the hierarchical materials and their properties. Section 1.2 presents the role of structural optimization in the design of materials. Also is presents the AM as a platform to test the design of hierarchical materials. Section 1.3 presents the problem formulation and the motivation to conduct this research. Section 1.4 presents the objectives that must be accomplished.

Chapter 2: This chapter presents a state-of-the-art of Topology Optimization (TO) for the design of cellular materials, the different techniques, and the different strategies to guarantee the connectivity between multi-microstructures solids. In addition, some additive manufacture (AM) techniques are described.

Chapter 3: This chapter comprises the theoretical background of topology optimization to design optimized solid and porous solid objects. Section 3.1 describes the mathematical formulation of the topology optimization method, the sensitivity analysis, and the filtering function. Section 3.2 presents a variation of topology optimization called Solid Isotropic Material with Penalization (SIMP). Section 3.3 embraces the mathematical formulation of Porous Anisotropic Material with Penalization (PAMP) method to design porous solid objects as a multiscale approach for single material and their extension to multi-material. Section 3.4 discusses the homogenization of porous material as a method to obtain the equivalent mechanical properties of the cell. Finally, section 3.5 shows two methods widely used in literature to ensure the connectivity between different cells in hierarchical solid with different kinds of microstructures.

Chapter 4: This chapter presents the motivation of the proposed approach to ensure the connectivity between cells on topologically optimized multi-region porous solids, the mathematical formulation and the advantages and drawbacks of the method also are analyzed.

Chapter 5: In this chapter, the numerical results for MBB-beam and L-shape problems are presented using two methods to design porous material including the 
approach proposed in Chapter 4, which are evaluated and compared. In addition, the verification of the code implemented and a comparison between 3D printed samples and FEM analysis are presented. Chapter 6 contains the main conclusions and suggestions for future work. Finally, Chapter 7 contains the references used in this text. 


\section{2 Literature Review on Multiscale Topology Optimization}

The next sections contain a review of the recent works that have been proposed for the design of architected material and some AM techniques. Here is a summary of the different techniques, methods, and intrinsic problems that serve as a background for this thesis.

\section{1}

\section{Design of Porous Solid}

The design of porous solids can be roughly divided into two main paths. The single-scale and the multiscale design. On the following will be presented

\subsection{1}

\section{Single-Scale Method}

One way to design a porous solid in a single-scale is just to fill the entire body with a prescribed pattern or cell, for example: Foam [66] (see Figure 2-1 a) or a grid pattern $[67,68]$ (see Figure 2-1 b). Another way is to use hybrid approaches, where the prescribed cell fills the body taking into consideration the solution of an optimization process. Lin et al. [69] use an optimization algorithm based on the concept of honeycomb-cell structure (see Figure 2-1 c). Jun et al. [40] proposed the use of a self-support geometric structure. The method consists of the split of a rhombic base form by means of TO in order to enhance the mechanical stiffness, static stability (see Figure 2-1 d), and buoyancy. 


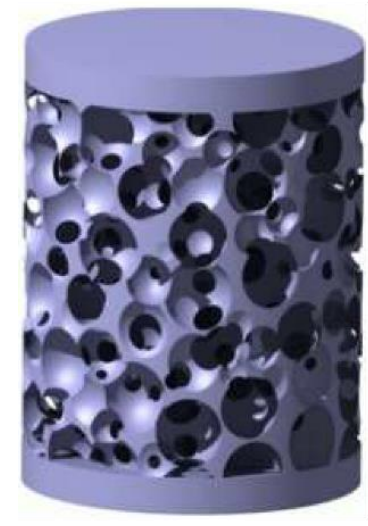

a)

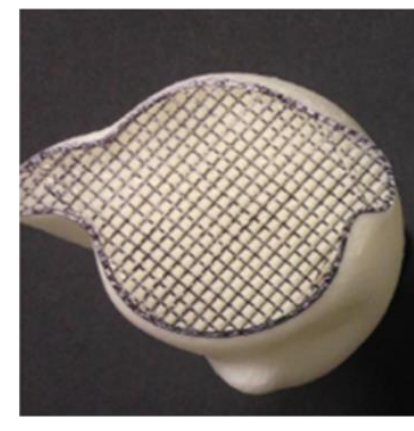

b)

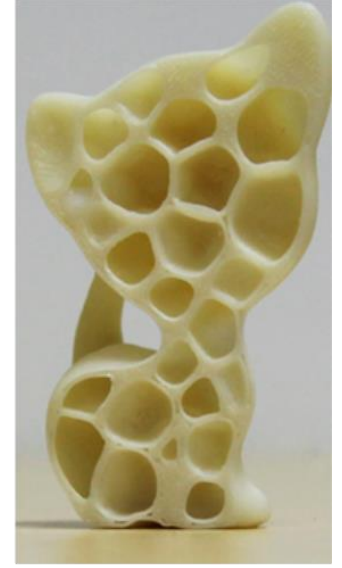

c)

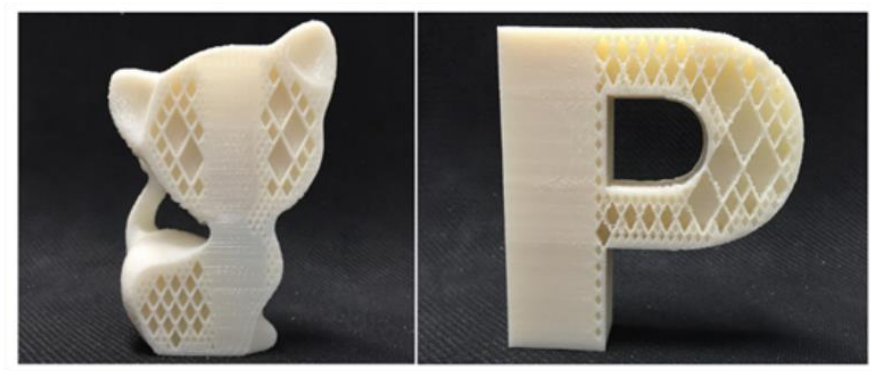

d)

Figure 2-1 - a) Foam infill [66]. b) Grid pattern infill [67, 68]. c) Honeycomb-cells infill [69]. d) Rhombic infill [40].

The literature is plenty of examples of single-scale methods that perform a TO and then fill the density output with a prescribed cellular shape, commonly called Variable-density [70-74]. Panesar et al. [70] introduced different variations, such as "intersected lattice", "Graded Lattice", and "Scaled Lattice" (see Figure 2-2 a). The Variable-density approach can be used to maximize diverse objectives such as the shear performance [75] or the natural frequency [76, 77]. Primo et al. [78] present a hybrid approach where different parts of the TO solution are replaced by the microstructures (see Figure 2-2 b). Han et al. [79] use a size gradient method to generate a non-uniform lattice structure with a distribution of different sizes of unit cells (see Figure 2-2 c). 


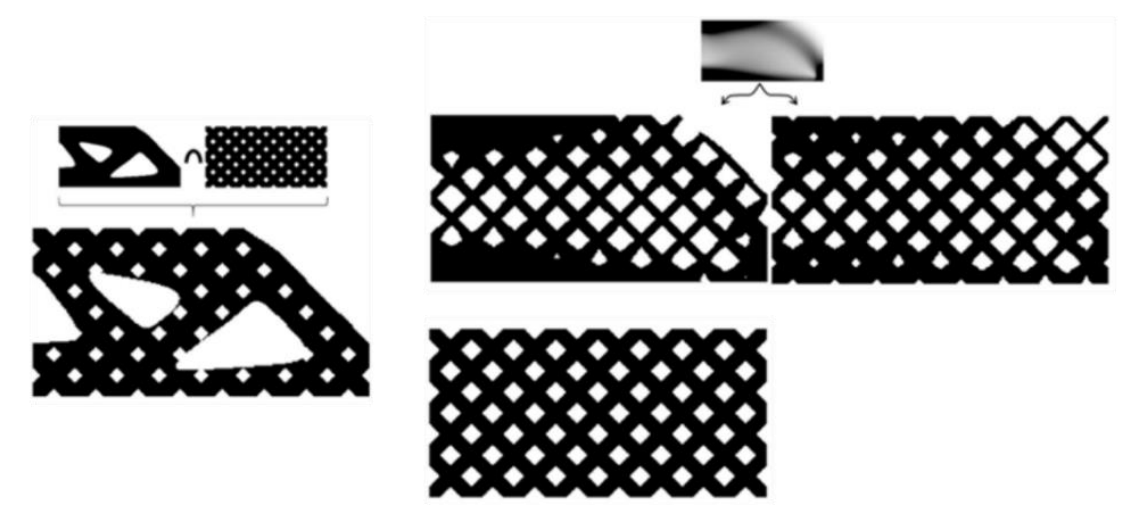

a)

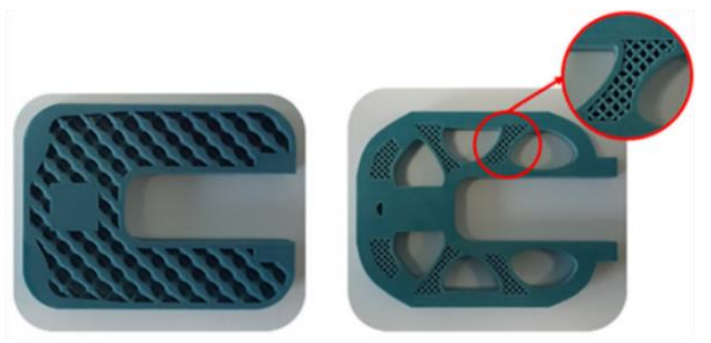

b)

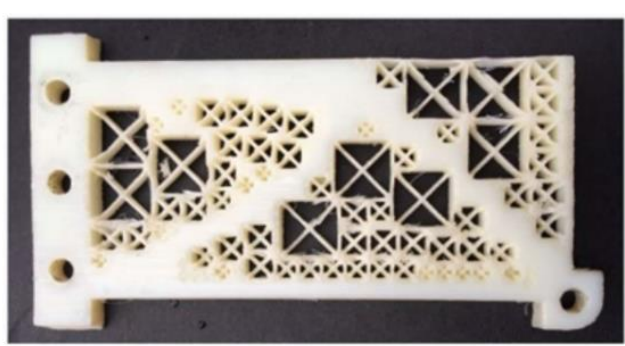

c)

Figure 2-2 - a) MBB-beam Lattice structures [70]. b) hybrid Variable-density with lattice [78]. c) Cantilever beam with nonuniform lattice [79].

Wu et al. [80] presented an approach to generate a bone-like porous material by regulating the local distribution and aligning the material to accommodate the mechanical loads in an optimized manner, as illustrated in Figure 2-3 a). Schumacher et al. [59] created different and independent families of microstructural cells for different volume fractions (Figure 2-3 b). These families of microstructural cells are projected into a metamaterial space (Figure 2-3 c) which permits analyze potential overlapping characteristics. Then, the cells with similar behavior (close in space) can be chosen to guaranteeing connectivity between them. This technique is used to generate flexible objects in 3D printing (Figure 2-3 d). 

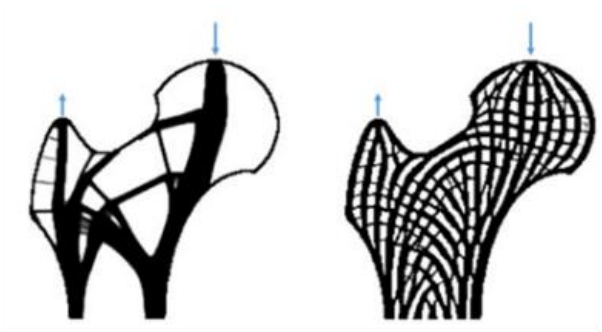

a)
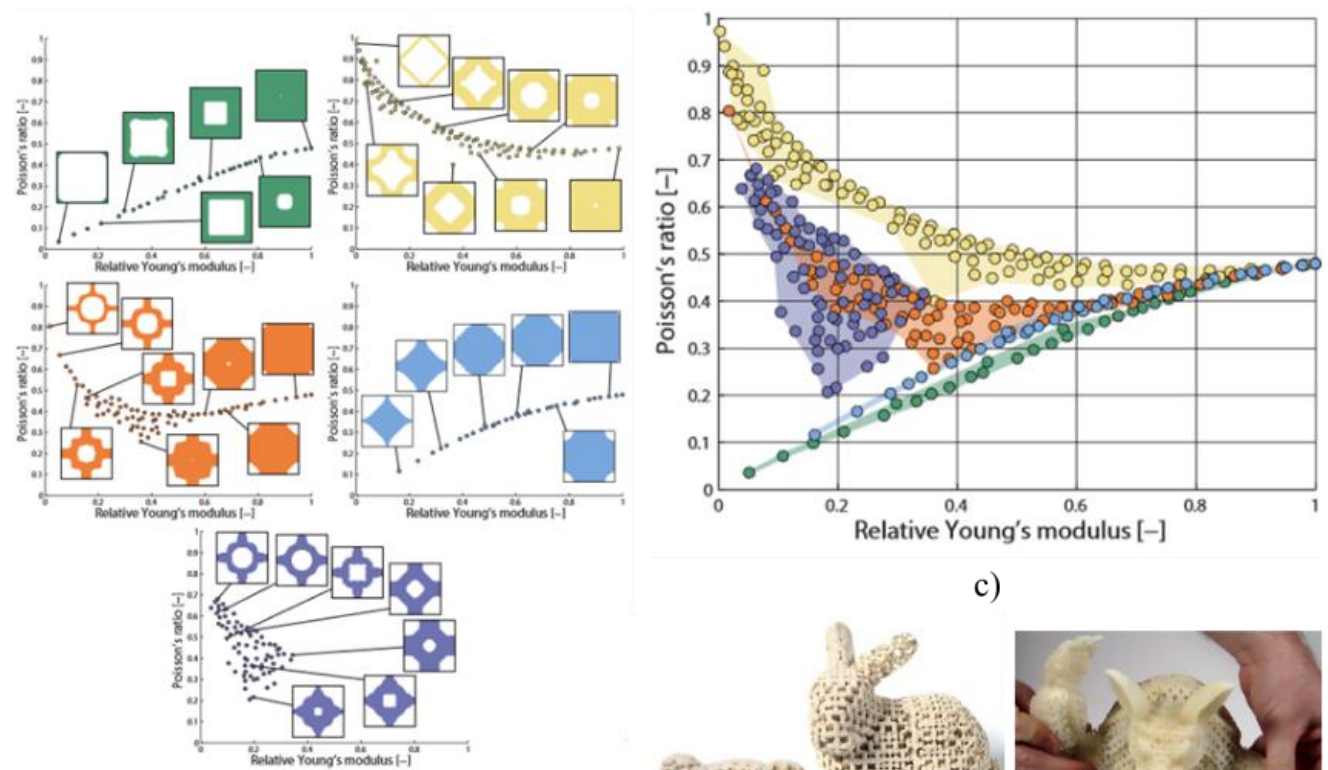

c)

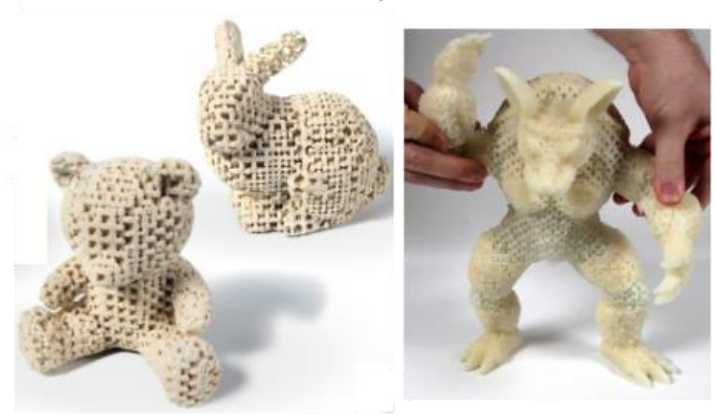

d)

Figure 2-3 - a) Porous bone-like solution [80], b-d) Microstructures to Control Elasticity [59].

\subsection{2}

\section{Multiscale Method}

The second way to design a porous solid is the multiscale approach. In this approach, two scales are considered, the macroscale and the microscale. The macroscale defines the optimal spatial material layout distribution and finds the optimal local use of the cellular material at the microscopic scale. To deal with different scales, the optimization needs to link/characterize the material properties (i.e., homogenization [32]), The multiscale design can be used to generated homogeneous $[44,50,81,82]$ or heterogeneous [51-53, 83] solids. Hence, it can be divided into two types [84]. The first type only uses a single microstructure 
throughout the domain (homogeneous) and the second one uses multiple microstructures in different regions on the solid (heterogeneous).

\subsubsection{1}

\section{Single Material or Single Cell}

Liu et al. [44] introduced the Porous Anisotropic Material with Penalization (PAMP). Figure 2-4 shows a macro structural topology (a) and the microstructural topology (b) of which is composed. The method was based on the SIMP and was proposed to optimize the system compliance. Other works then extend it to thermal load [85, 86] and fundamental frequencies [82], among others [87].

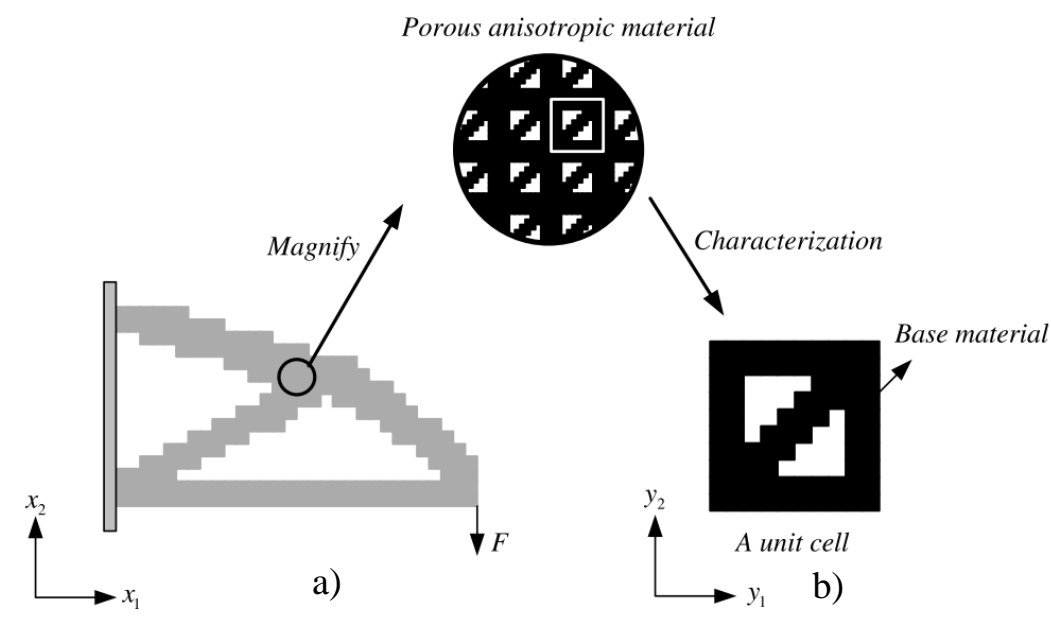

Figure 2-4 - A structure composed of a porous anisotropic material [44].

Yan et al. [45] use topology optimization with BESO algorithm (Bi-directional Evolutionary Structural Optimization) [46, 47] (Figure 2-5 a). Wang et al.[48] proposed a framework using Level Set Method (LSM) (Figure 2-5 b). Chen et al. [49] introduced the Moving Iso-Surface Threshold (MIST) to the concurrent optimization (Figure 2-5 c). In [50], the authors presented Isogeometric Analysis (IGA,[88]) for TO also called Isogeometric TO (ITO). 


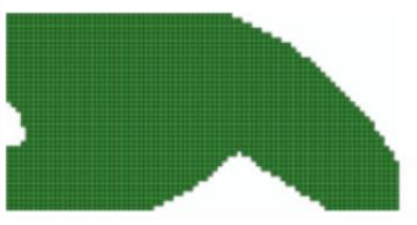

a)

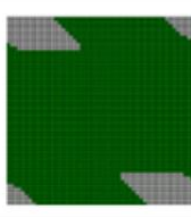

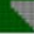

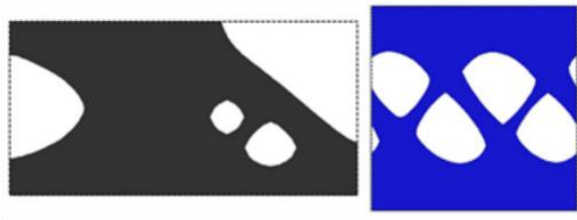

b)
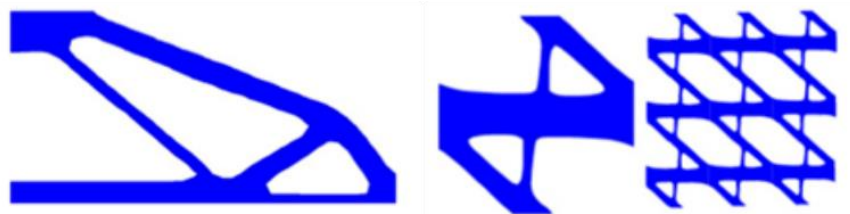

c)

Figure 2-5 - a) TO with BESO [45]. b) TO using (LSM) [48]. c) TO using MIST [49].

Despite the existence of a variety of methods, to our knowledge, only one is open source. The work presented in [84] is an open source implementation of concurrent topology optimization for 2D and 3D lattice material written in MATLAB. The method uses a modification of SIMP and the Energy-Based Homogenization Method (EBHM).

\subsubsection{2}

\section{Multi-material or Multi-region}

The second type of design porous solid in a multiscale TO problem employs multi-microstructures or materials. Xia et al. [28] use a $\mathrm{FE}^{2}$ non-linear analysis with high computational cost (Figure 2-6 a). Sivapuram et al. [51] decomposed the problem, allowing parallel computing (Figure 2-6 b). Li et al. [52], applied the topology optimization process with multi-patch microstructures by a parametric LSM as shown in Figure 2-6 c. 


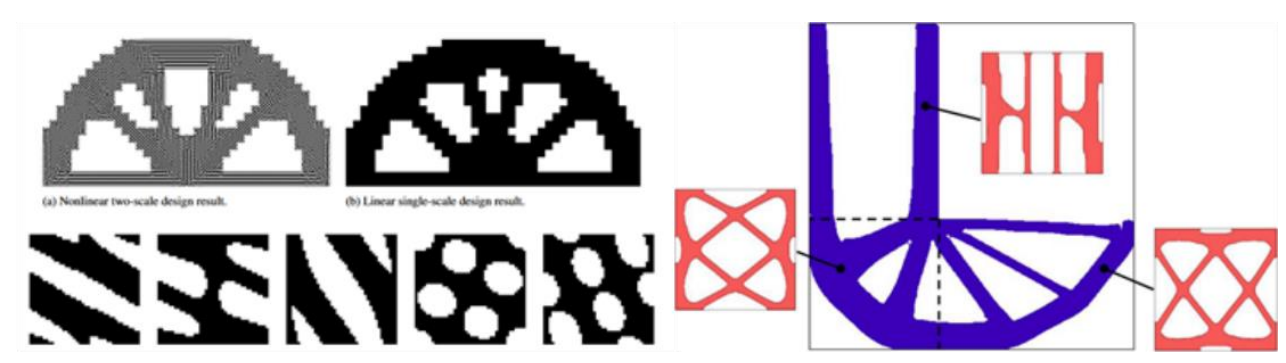

a)

b)

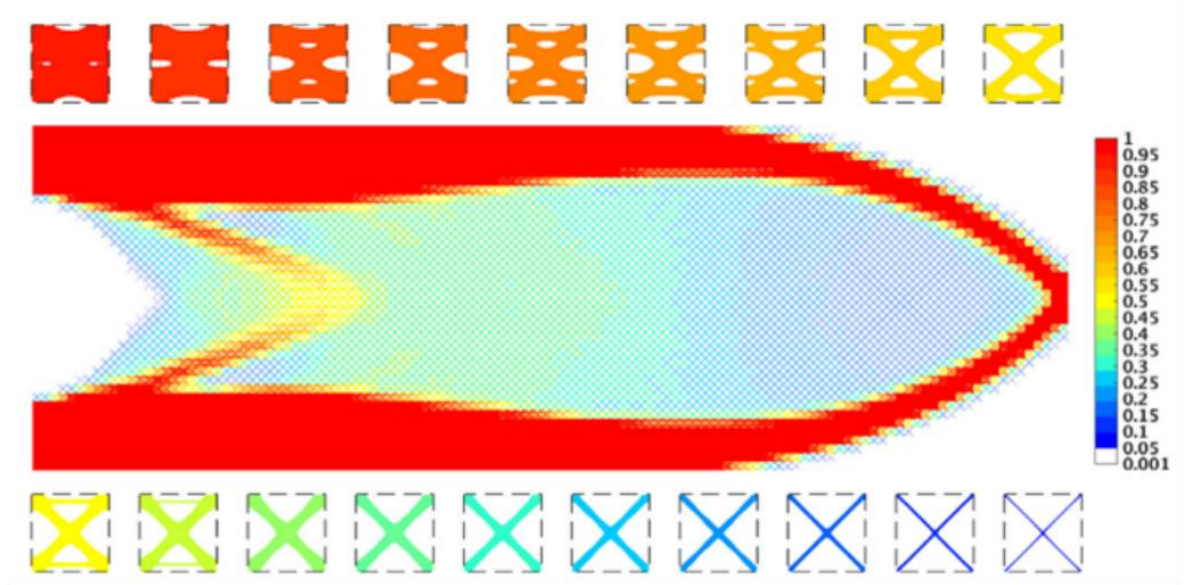

c)

Figure 2-6 - a) TO with non-linear analysis [28].b) TO decomposed with LSM [51] c) TO with multi-patch microstructures [52].

The work done by Gao et. al. [89] presents a multiscale topology optimization method for multiples design regions, which considers three design elements: the macrostructural topology, the topological microstructures, and their overall distribution at the macrostructure (Figure 2-7 a). Wang et al. [90] use PAMP to design non-uniform microstructures (Figure 2-7 b). The work proposed by Zhang et al. [60] consists of concurrent topology optimization as sectorization of the solid as layer-wise (Figure 2-7 c). In [91] the PAMP method is extended to multiples microstructures under random load. 


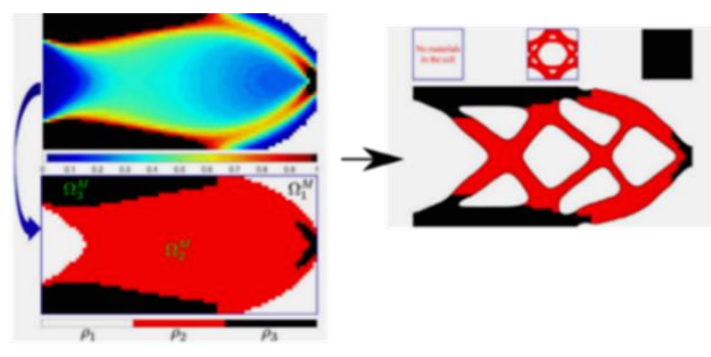

a)

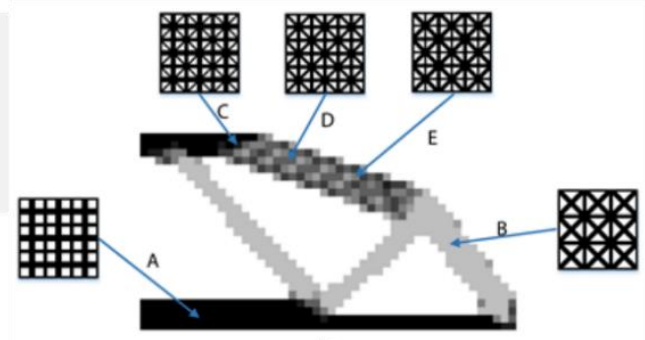

b)

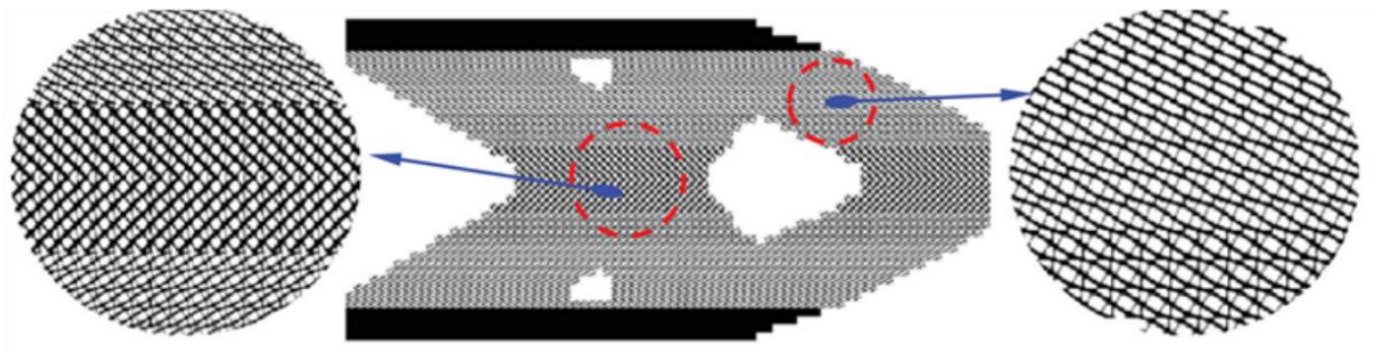

c)

Figure 2-7 - a) cells by density distribution [89]. b) PAMP and non-uniform microstructures [90]. c) TO with layer-wise sectorization [60].

Rodrigues et al. [53] described a hierarchical optimization procedure based on the SIMP method (Figure 2-8 a) which were later extended to 3D problems in [83]. $\mathrm{Xu}$ et al. [54] presented a multi-resolution topology optimization (MTOP) scheme with IGA to obtain a high-resolution representation for complex geometries. The scheme was implemented with two different mesh discretization, and a non-uniform rational basis spline (NURBS) to do a projection scheme and compute the density variables (Figure 2-8 b). The work presented in [55] is a concurrent optimization process wherein the macroscale is used the Velocity Field Level Set method (VSFL) [57] and for the microscale is used the SIMP method.

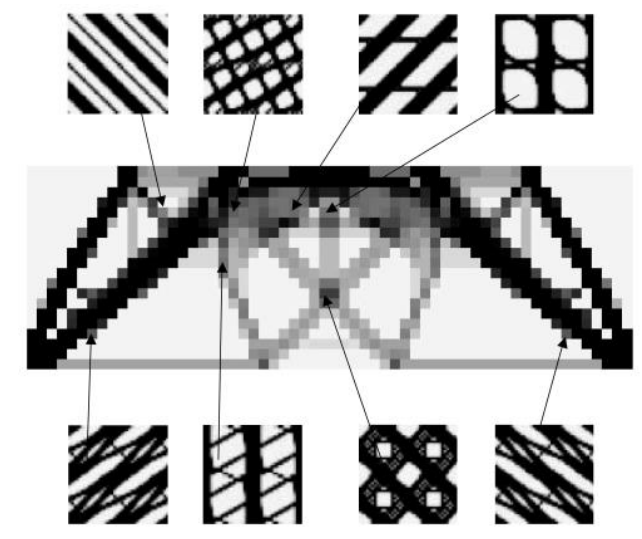

a)

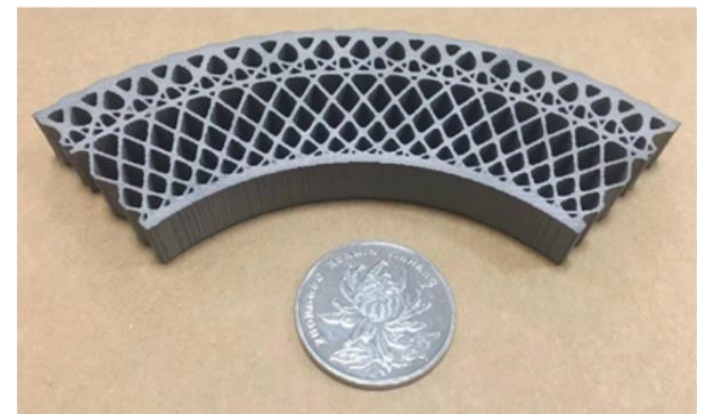

b)

Figure 2-8 - a) hierarchical optimization [53]. b) MTOP [54]. 
Different from the first case where the periodicity of the cells ensures the selfcompatibility, the multiscale TO with multiple types of cells lacks of connectivity between cells and this becomes a big issue in porous solids design, since there may be internal penetration between cells and a potential stress concentration. Figure 2-9 shows the TO solution for two different problems with their respective microstructures, the green circles denoted the

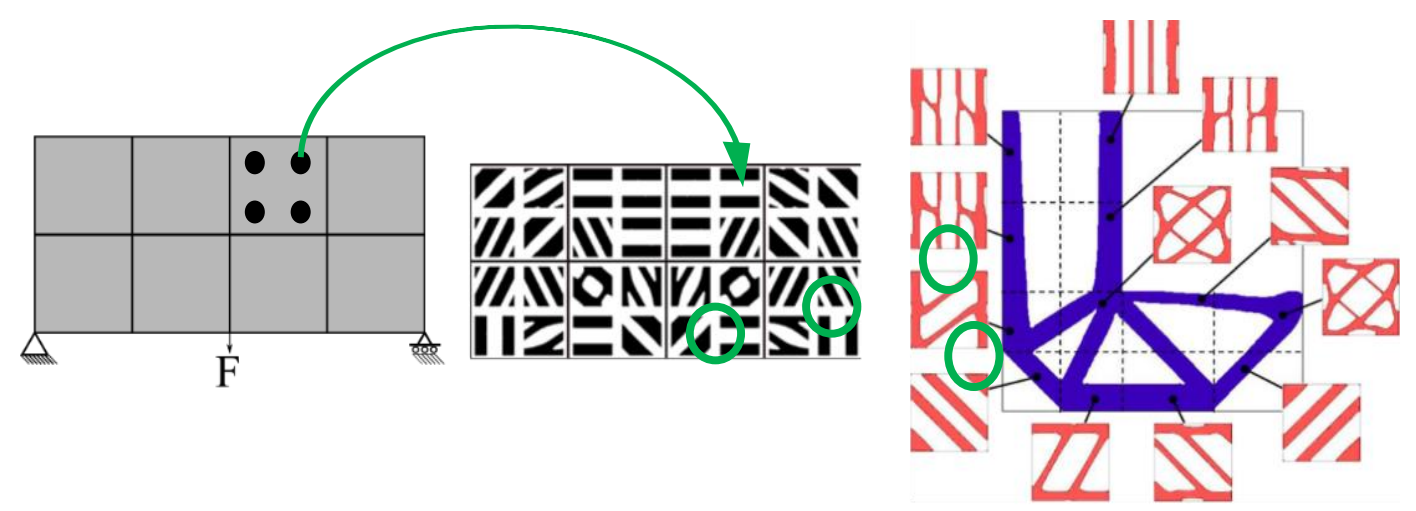

Figure 2-9 - Microstructure self-compatibly issues, adapted from [28, 51].

To tackle this connectivity issue, different strategies have been proposed [54, 58, 60-64]. Du et al. [58] introduced the Connectivity Index (CI) constraint in order to improve the connection. Zhang el at. [60] divided the design region into multiple layers to generate a smooth connection, but it does not ensure the full connectivity between layers. Zhou et al. [62] proposed three approaches to establish the connection: a) Kinematic Connective constrain (KC), b) Pseudo-Load (PL), and c) Unified Formulation (UF) with nonlinear diffusion. Wang et al. [61] used a shape metamorphosis approach [92], however, this implicit control cannot guarantee fully connectivity (Figure 2-10 a). The work done in [63] uses BESO method to design a Functionally Graded Material (FGM) [62, 93] and a filter to smooth the connection between adjacent cells, this method can create discontinued members (Figure 2-10 b) which can lead to stress concentrations. Garner et al. [64] introduced "compound cells" (Figure 2-10 c) to perform a "global" density filter to connect the cell along the x-direction, similar to [63]. Although the authors claim that, the method can be adapted for more than one direction, the scheme presents poor performance. The most commonly used strategies used are to modify the whole set of cells that compose the solution $[52,55,58,61,62]$ and create a predefined shape 
with self-compatibility [40, 70, 75-79], which restring the design space of the solution [65].

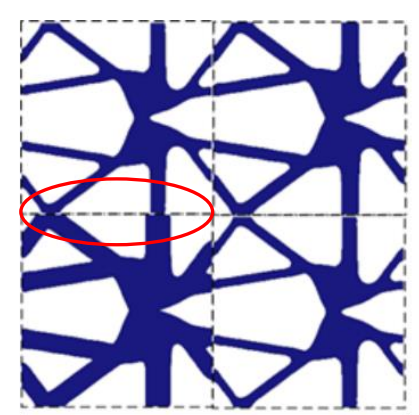

a)

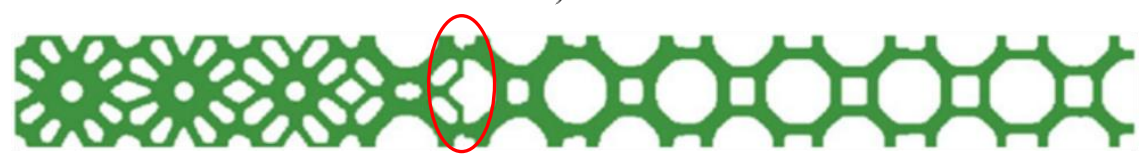

b)

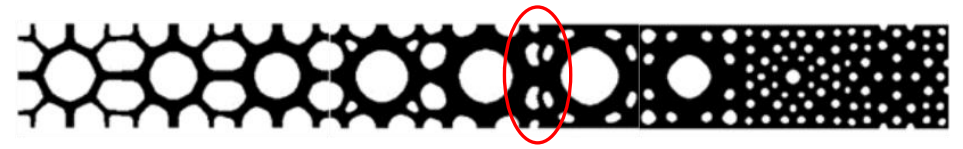

c)

Figure 2-10 - a) Shape metamorphosis [61]. b) BESO FGM [63]. c) Compound cells [64].

\section{2 \\ Additive Manufacturing (AM)}

The fine and high complexity forms that can be obtained in TO were considered prohibitive solutions for the manufacturing methods until the arrival of additive manufacturing (AM). This process, commonly called 3D printing, allows to build a physical object adding material layer-upon-layer from a computational 3D model. The most used materials are plastic, paper, resin, and metal. In the following, the most used methods of AM that are compatible with the manufacturing of microstructures will be briefly explained below.

\subsection{1}

\section{Stereolithography}

The Stereolithography Apparatus (SLA) is a common process used in digital manufacturing, being one of the first methods in AM based on the solidification of a liquid material (stored inside of a container), with the help of a light beam that draws a pre-programmed shape. The liquid is composed of an acrylic monomer, 
oligomer, and a photochemical compound [94]. When the solidification process is completed at $75-85 \%$ it is exposed to the light and finishes in the absence of the laser [95]. At the end of the process, the support material is broke up and removed, later the prototype is placed in an oven to complete the curing.

The manufactured method can take hours or even days, without taking into account the post process of painting and polish. An advantage of this method is the precision, reaching up to $432 \mathrm{~nm}$ in the micro and nanostereolithography [96], which is used in Microelectromechanical Systems (MEMS) [97, 98]. The versatility of this method allows to use in the aerospace industry [99], the arms industry, automotive [100], electronics, toys, industrial equipment, medical applications as surgery, and odontology [101-104]. Figure 2-11 shows a) a bull made by microstereolithography and b) a micro-oscillator.

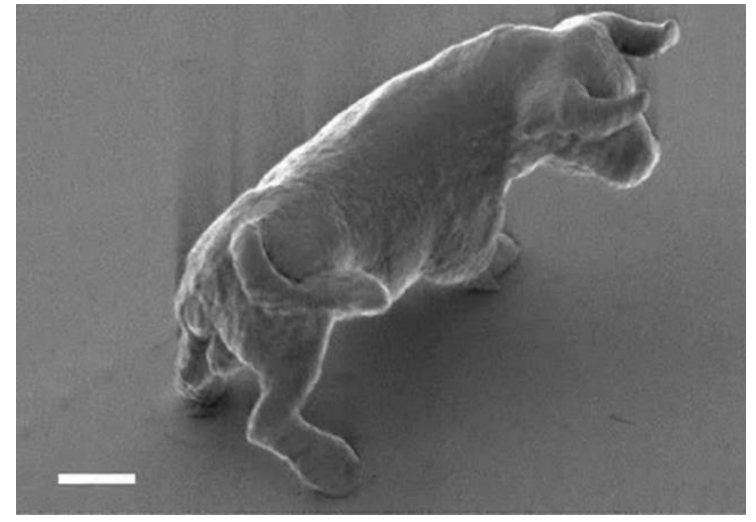

a)

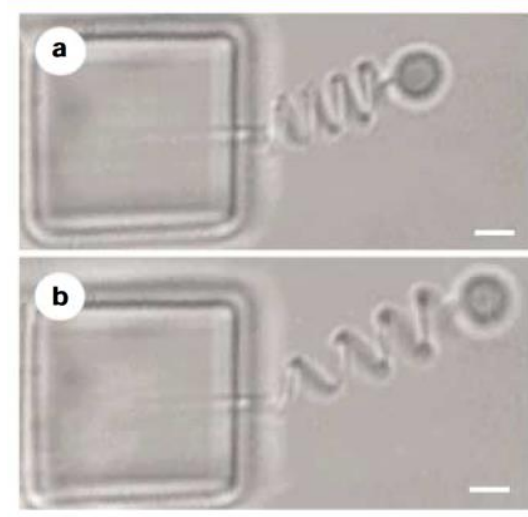

b)

Figure 2-11 - a) Microbull made by two-photon microstereolithography. b) micro-oscillator system. Scale bar $2 \mu m$ [105].

\subsection{2}

\section{Selective Laser Sintering}

The selective laser sintering (SLS) consists of the fusion and solidification of metallic or not metallic powder (SLM- selective laser melting, DMLS direct metal laser sintering [106]). The process is similar to the SLA, but in this case, it is used thermoplastic powder or a thermoplastic binder.

The SLS consists of three platforms, one is the solid's container, and the other two supplies the material that will be melted with a laser beam and subsequently cooled. When the process ends, the solid is removed from the platform to further apply a post-processing step that can be a thermic treatment or a micro rotatory tool 
and file [107]. The process is more complex when compared to the SLA (the final object is rough and demand post-process), but as an advantage, the object can be functional. This method is compatible with micro manufacture as MEMS [108, 109], injection molds [106, 110], and other applications as exoskeletons [111]. Figure 2-12 shows a two-component printed object (Copper-Tin).

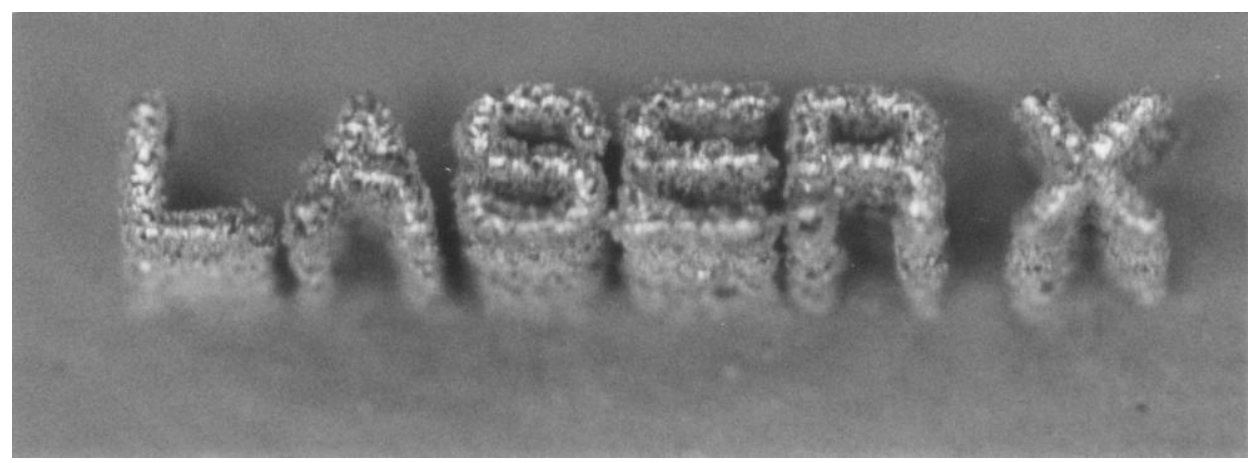

Figure 2-12 - Two-component liquid-phase sintering of $\mathrm{Cu}-\mathrm{Sn}$. Line width=470 $\mu \mathrm{m}$ [108].

\subsection{3 \\ Fused Deposition Modelling}

Fused Deposition Modeling (FDM) also called Fused Filament Fabrication (FFF) is one of the more popular methods of 3D printing. The method use fused material to deposit tiny layers. Normally is a gantry crane device, similar to those used in the industry these motor-assisted machines let the load move on all axes. The material can be thermoplastic or wax in thread form [94]. When it is finished the tridimensional object and the supports can be removed with sandpaper or dissolved in a chemical solution when printed with more of one material [112]. Figure 2-13 shows three FDM printed objects with bone-like porous infill.

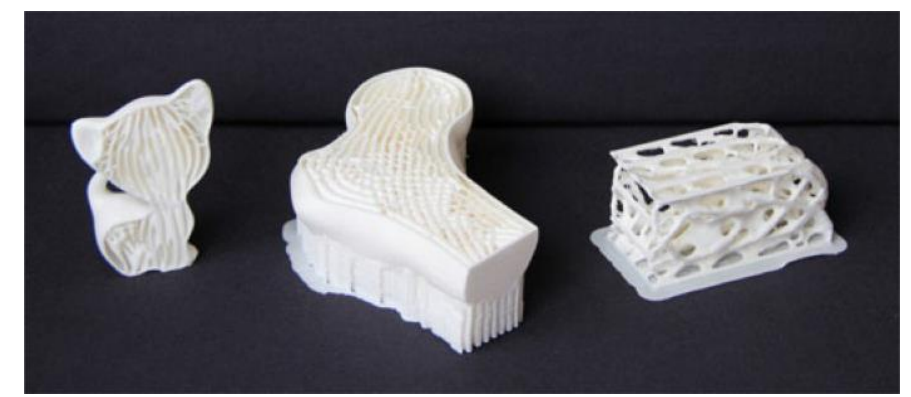

Figure 2-13 - FDM bone-like porous infill [80]. 
The method permits to quickly create small parts and use a variety of material such as ABS, ABSi, wax, PLA, Flexi PLA, Nylon, Benlay, Laywood, Laybrick, Polyphensulfone (PPFS), PC, PC-ISSO, Chocolate, PTZ, aluminum Oxide, stainless steel, Hydroxyapatite and iron $[113,114]$, and a high variety of composite materials [115]. The method is used in almost any industry, such as aerospace [116118], medical equipment [119-124], architecture [125-127], food industry [128131], human organs for study and transplantation [132-136], toys [137, 138] and in general commerce.

As can be seen, these AM's techniques have the versatility to manufacture objects in a wide range of sizes achieving the micrometers length. They can also be used to produce topologically optimized objects with a range of materials. 


\section{3 \\ Multiscale Topology Optimization}

This chapter presents the theoretical background used in this study. First Topology Optimization and the Solid Isotropic Material with Penalization (SIMP) method are described. In the following, the multiscale design using Porous Anisotropic Material with Penalization (PAMP) method single and multi-region (materials), is introduced. Next, the homogenization theory is discussed and finally, some approaches that have been proposed to preserve the connectivity between adjacent cells are detailed.

\section{1}

\section{Topology Optimization (TO)}

Considering a mechanical element as a body that occupies a domain $\Omega$ in $\mathbb{R}^{d} d=$ 2,3 ; that is subject to a set of constraints, such as prescribe displacement in a nonzero measurement surface $\boldsymbol{\Gamma}_{D}$ and non-zero traction forces $\mathbf{t}$ is applied at $\boldsymbol{\Gamma}_{N}$ (see Figure 3-1). The principal objective of topology optimization is to find the optimal material distribution $(\omega)$ that can minimize or maximize a certain objective.

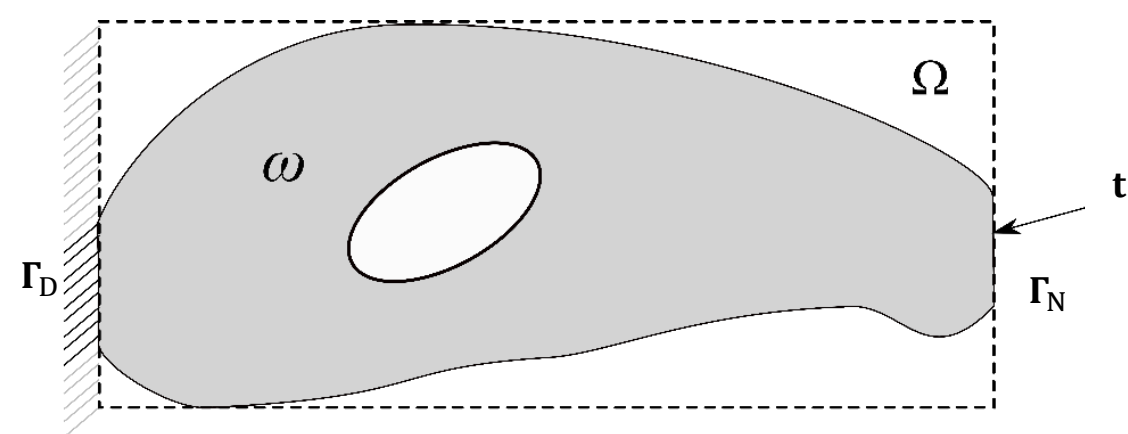

Figure 3-1 - Illustration of Domain and boundary conditions. 
The mathematical formulation of the TO reads as $[16,17]$

$$
\begin{array}{cc}
\min & c(\boldsymbol{\rho})=\mathbf{U}^{\mathbf{T}} \mathbf{K} \mathbf{U} \\
\boldsymbol{\rho} & \frac{V(\boldsymbol{\rho})}{V_{\mathbf{0}}}=V_{f} \\
& \mathbf{K U}=\mathbf{F} \\
& 0 \leq \boldsymbol{\rho} \leq 1
\end{array}
$$

where $c(\boldsymbol{\rho})$ is the compliance (in this case the objective function), $\mathbf{K}$ is the global stiffness matrix, $\mathbf{U}$ and $\mathbf{F}$ are the global displacement and force vectors, respectively. $V_{0}$ and $V(\boldsymbol{\rho})$ are the volume of the design domain and volume function, respectively, $V_{f}$ is the prescribed volume fraction, and $\boldsymbol{\rho}$ are the vector design variables. The global stiffness $\mathbf{K}$ can be obtained as

$$
\mathbf{K}=\sum_{e=1}^{N} \mathbf{K}_{e}
$$

Where $N$ is the number of elements, and $\mathbf{K}_{e}$ is the stiffness matrix for the e-th element denoted as follow

$$
\mathbf{K}_{e}=\int_{\boldsymbol{\Omega}_{e}} \mathbf{B}_{e}^{T} \mathbf{D}_{e}\left(\rho_{e}\right) \boldsymbol{B}_{\mathrm{e}} d \mathrm{~V}
$$

where $\mathbf{B}_{e}$ is the strain/displacement matrix and $\mathbf{D}_{e}$ is the element constitutive matrix.

Figure 3-2 shows the flowchart of the TO process. The first step is to initialize the Finite Element Method (FEM) and set the initial guess of the design variables $\boldsymbol{\rho}$, followed by the evaluation of the Finite Elements (FE) analysis and the objective function. Later the sensitivity analysis is evaluated, the restriction function and the filter are applied, and then the material is redistributed. This process is repeated until either the maximum number of iterations is exceeded or the change in design variables is smaller than the prescribed tolerance. 


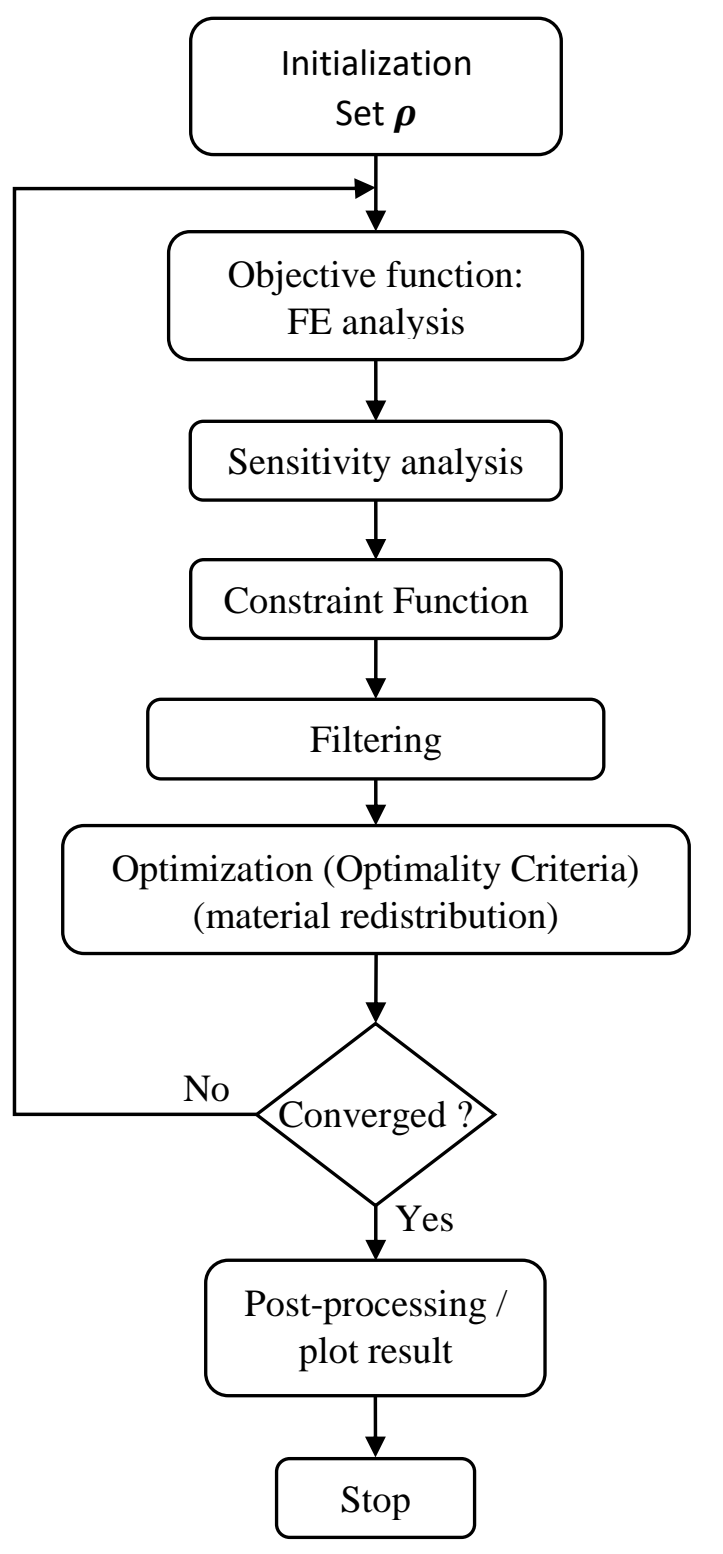

Figure 3-2 - Flowchart of the topology optimization process.

\subsection{1}

\section{Sensitivity Analysis}

The sensitivity analysis allows us to measure the variation of performance with respect to the design variables [10]. In this analysis, structural compliance can be written as

$$
c=\mathbf{F}^{T} \mathbf{U}
$$


Differentiating the two sides with respect to the design variables, we have

$$
\frac{\partial c}{\partial \rho}=\frac{\partial \mathbf{F}^{\mathbf{T}}}{\partial \rho} \mathbf{U}+\mathbf{F}^{\mathrm{T}} \frac{\partial \mathbf{U}}{\partial \rho}
$$

and deriving the equilibrium equation $\mathbf{K U}=\mathbf{F}$

$$
\frac{\partial \mathbf{F}}{\partial \rho}=\frac{\partial \mathbf{K}}{\partial \rho} \mathbf{U}+\mathbf{K} \frac{\partial \mathbf{U}}{\partial \rho}
$$

isolating $\partial \mathbf{U} / \partial \rho$

$$
\frac{\partial \mathbf{U}}{\partial \rho}=\mathbf{K}^{-1}\left(\frac{\partial \mathbf{F}}{\partial \rho}-\frac{\partial \mathbf{K}}{\partial \rho} \mathbf{U}\right)
$$

and replacing equation (7) in equation (5) we have

$$
\frac{\partial c}{\partial \rho}=\frac{\partial \mathbf{F}^{T}}{\partial \rho} \mathbf{U}+\mathbf{F}^{T} \mathbf{K}^{-\mathbf{1}}\left(\frac{\partial \mathbf{F}}{\partial \rho}-\frac{\partial \mathbf{K}}{\partial \rho} \mathbf{U}\right)
$$

and knowing that the force does not depend on the design variables, the final expression of the sensitivity of the compliance function with respect to $\rho$ is

$$
\frac{\partial c}{\partial \rho}=-\mathbf{F}^{T} \mathbf{K}^{-1} \frac{\partial \mathbf{K}}{\partial \rho} \mathbf{U}=-\mathbf{U}^{T} \frac{\partial \mathbf{K}}{\partial \rho} \mathbf{U}
$$

\subsection{2}

\section{Optimality Criteria Method}

The Optimality Criteria method (OC) is one of several different approaches used to solve the OT problems. Its development for continuum problems can be dated back to Taylor et al. [139] where is present a method fir the optimal design of sandwich structures, and the use in continuum design problems also can be found in [140-142]. OC is a heuristic updating scheme. 
Following [143, 144], the TO problem (1) can be solved using OC and formulated as

$$
\begin{aligned}
& \rho_{e}^{\text {new }} \\
& =\left\{\begin{array}{cc}
\max \left(0, \rho_{e}-m\right) & \text { if } \rho_{e} B_{e}^{\eta} \leq \max \left(0, \rho_{e}-m\right) \\
\min \left(1, \rho_{e}+m\right) & \text { if } \rho_{e} B_{e}^{\eta} \geq \min \left(1, \rho_{e}+m\right) \\
\rho_{e} B_{e}^{\eta} & \text { otherwise }
\end{array}\right.
\end{aligned}
$$

where $m$ is a positive move limit, $\eta$ is a damping coefficient equals $1 / 2$, and the value $B_{e}$ is the optimality condition described as

$$
B_{e}=\frac{-\partial c / \partial \rho_{e}}{\lambda^{\partial \mathrm{V}} / \partial \rho_{e}}
$$

Being $\lambda$ the Lagrangian multiplier found by means of a bisection algorithm and based on the assumption that each element has unit volume

$$
\frac{\partial \mathrm{V}}{\partial \rho_{e}}=1
$$

\subsection{3}

\section{Filtering}

To ensure the solution of topology optimization and prevent some problems as a checkerboard pattern $[11,145,146]$; the sensitivity filter is commonly used as a restriction in the design. The sensitivity filter modifies the sensitivities $[10,143$, 144] as follows

$$
\frac{\widehat{\partial c}}{\partial \rho_{e}}=\frac{1}{\max \left(\gamma-\rho_{e}\right) \sum_{i \in N_{e}} H_{e i}} \sum_{i \in N_{e}} H_{e i} \rho_{i} \frac{\partial c}{\partial \rho_{i}}
$$

where $\gamma$ is a small positive number to avoid division by zero, $N_{e}$ is the set of elements that are at a distance from center to center $\Delta(e, i)$ less than the value of the radius of the filter $r_{\text {min }}$, and the weight factor $H_{e i}$ defined as follows

$$
H_{e i}=\max \left(0, r_{\min }-\Delta(e, i)\right)
$$




\section{2}

\section{Solid Isotropic Material with Penalization (SIMP)}

In SIMP [16, 147-149] interpolation is applied a penalization to the design variables $(\rho)$ to eliminate the intermediate values and recover the binary (blackwhite) nature of the problem $[16,17,148]$. For penalization values $p>1$ the intermediate densities are seen as unfavorable because the stiffness obtained is less than the volume of the material forcing the elimination of these ensuring a blackwhite solution (Solid material and void). So, the stiffness of each element $e$ depends on its density $\rho$ and following the modified approach [150] is calculated as

$$
E_{e}\left(\rho_{e}\right)=E_{\min }+\rho_{e}^{p}\left(E_{0}-E_{\min }\right) \rho_{e} \in[0,1]
$$

where $E_{\min }$ is Young's modulus of the Ersatz material, which is an approximation for void material [151] to prevent the singularity of the stiffness matrix, and $E_{0}$ is the Young`s modulus of the solid material. For higher values of $p$, the influence of the elements with intermediate density values $(\rho)$ decreases as shown in Figure 3-3. The horizontal axis is the density value and the vertical axis is the value of the density after the penalization is applied. The density variation for five different values of $p$ is shown. It is frequently advised to raise the power value in a continuous fashion $(p=1-4)$ through the computation until the design is finished [10]. 


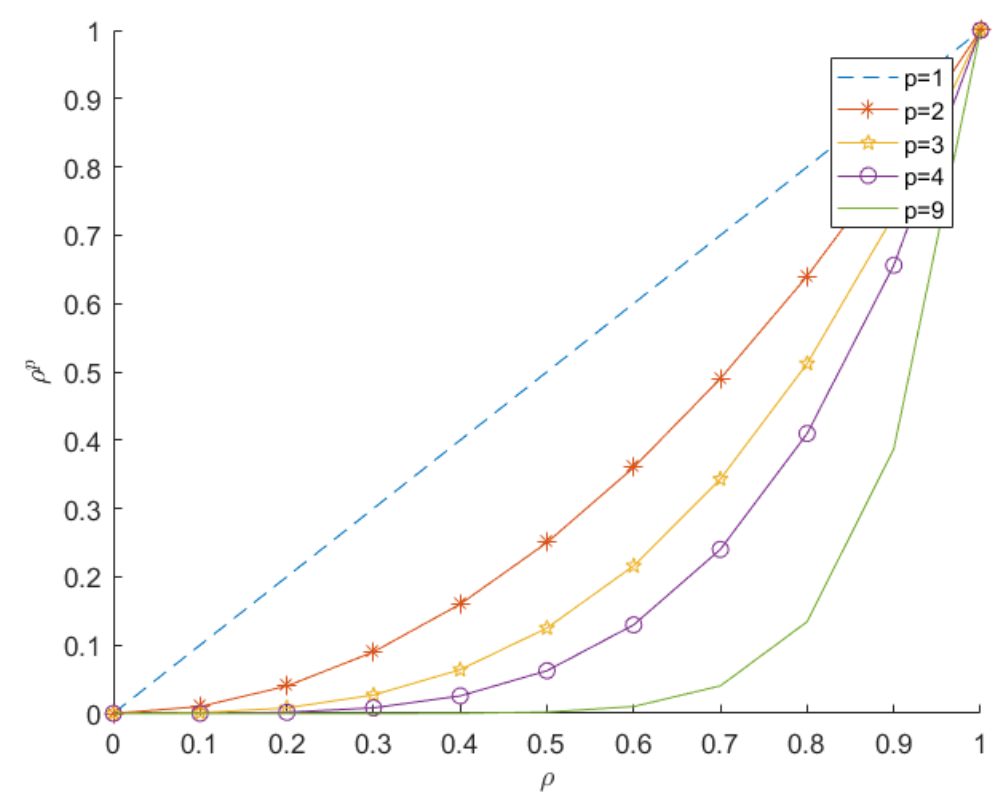

Figure 3-3 - Density $(\rho)$ against penalized density $\left(\rho^{p}\right)$ for different penalization factors $p$.

\section{3}

\section{Porous Anisotropic Material with Penalization}

The structure shown in Figure 3-4 is composed of a porous material with uniform microstructures, theses microstructures are made of a base material that can be of any type of solid material. Hence, the structure consists of "two types" of material, the solid (microscale), and the porous material (macroscale). To solve this problem, Liu et al. [44] proposed the Porous Anisotropic Material with Penalization (PAMP) approach, that different from SIMP, this tries to obtain a "grey-white" solution i.e. porous and void. Here the problem becomes concurrent and is divided into 2 parts. The first one is the microscale that needs to be interpreted or characterized to be used and the second one, the macroscale that must be optimally distributed in the design area. The microscale design can be characterized as a porous material by means of a homogenization method, using the smallest representative unit called "unit cell". 


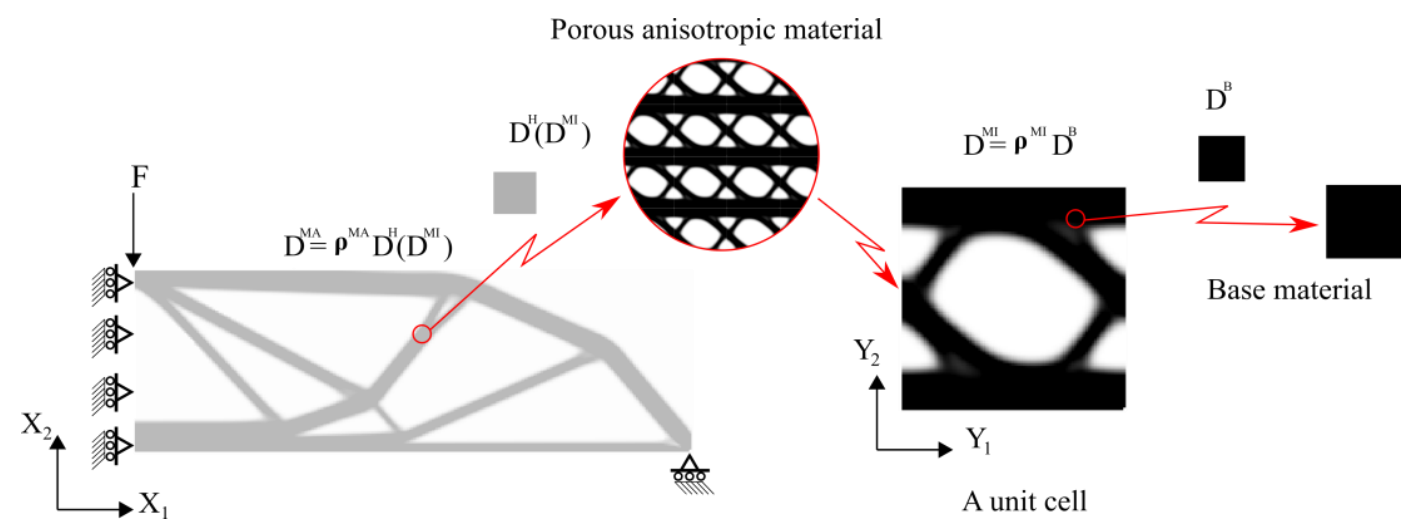

Figure 3-4 - A macrostructure composed of a porous anisotropic material.

To carry out the solution to this problem is necessary to define two classes of design independent variables, i.e. the macro density $\boldsymbol{\rho}_{M A}$ in the structural domain, and the micro density $\boldsymbol{\rho}_{M I}$ in a cell (see Figure 3-4). The mathematical formulation of the minimization problem reads as follows:

$$
\begin{array}{cc}
\min & c=\mathbf{F}^{T} \mathbf{U} \\
\text { subject to } & v=\frac{\rho^{P A M} \int_{\Omega} \boldsymbol{\rho}_{M A} d \Omega}{\left.A_{M A}, \boldsymbol{\rho}_{M I}\right]}=\bar{v} \\
& \rho^{P A M}=\frac{\int_{Y} \boldsymbol{\rho}_{M I} d Y}{A_{M I}}=\bar{v}_{M I} \\
& 0 \leq \boldsymbol{\rho}_{M A} \leq 1, \quad 0 \leq \boldsymbol{\rho}_{M I} \leq 1 \\
\text { with } & \mathbf{K U}=\mathbf{F}
\end{array}
$$

where $\boldsymbol{\rho}_{M I}$ and $\boldsymbol{\rho}_{M A}$ are the density of micro and macroscale, respectively, $v$ is the relative volume that is smaller than the prescribed macroscale volume $\bar{v}, \rho^{P A M}$ is the relative density of the porous material that should be equal to the prescribed volume at the microscale $\bar{v}_{M I}, A_{M A}$, and $A_{M I}$ is the area of the macro and microscale design respectively.

Similar to SIMP, the elastic matrix at the microscale can be written as

$$
\mathbf{D}^{M I}=\rho_{M I}^{p_{M I}} \mathbf{D}^{B}
$$

where $\mathbf{D}^{B}$ is the elastic matrix of the base material and $p_{M I}$ is the penalization factor (see Figure 3-4). At the macroscale the material is porous anisotropic, therefore, the 
SIMP is not applied. However, the process is quite similar. Given a porous material with a homogenized elastic matrix $\mathbf{D}^{H}$ (for more details to how obtain this value, see section 3.4), a point with a density value $\rho_{M A}$, and a penalization value $p_{M A}$, the elastic matrix at the macroscale is calculated as

$$
\mathbf{D}^{M A}=\rho_{M A}^{p_{M A}} \mathbf{D}^{H}\left(\mathbf{D}^{M I}\right)
$$

therefore, to solve problem (16) the finite element analysis for the macroscale is formulated as follows

$$
\mathbf{K U}=\mathbf{F} .
$$

The element stiffness matrix $\mathbf{K}_{\boldsymbol{e}}$ is

$$
\mathbf{K}_{e}=\int_{\Omega_{e}} \mathbf{B}_{e}^{T} \mathbf{D}^{M A} \mathbf{B}_{e} d \mathrm{~V}
$$

$\mathbf{D}^{M A}$ is a function of $\mathbf{D}^{H}$, as shown in equation (18), being the link between scales because defines the effective material properties that depends on the microstructural configuration and can be calculated by means of a homogenization method.

Figure 3-5 shows a flowchart of the PAMP process. The first step is to initialize the FEM and the design variables for both macro and microscale. The second step is to evaluate the FE analysis and the objective function in the two scales. The next step is to apply the constrain functions and evaluate the sensitivity analysis also in both scales, and finally, the material is redistributed until the model converges. 


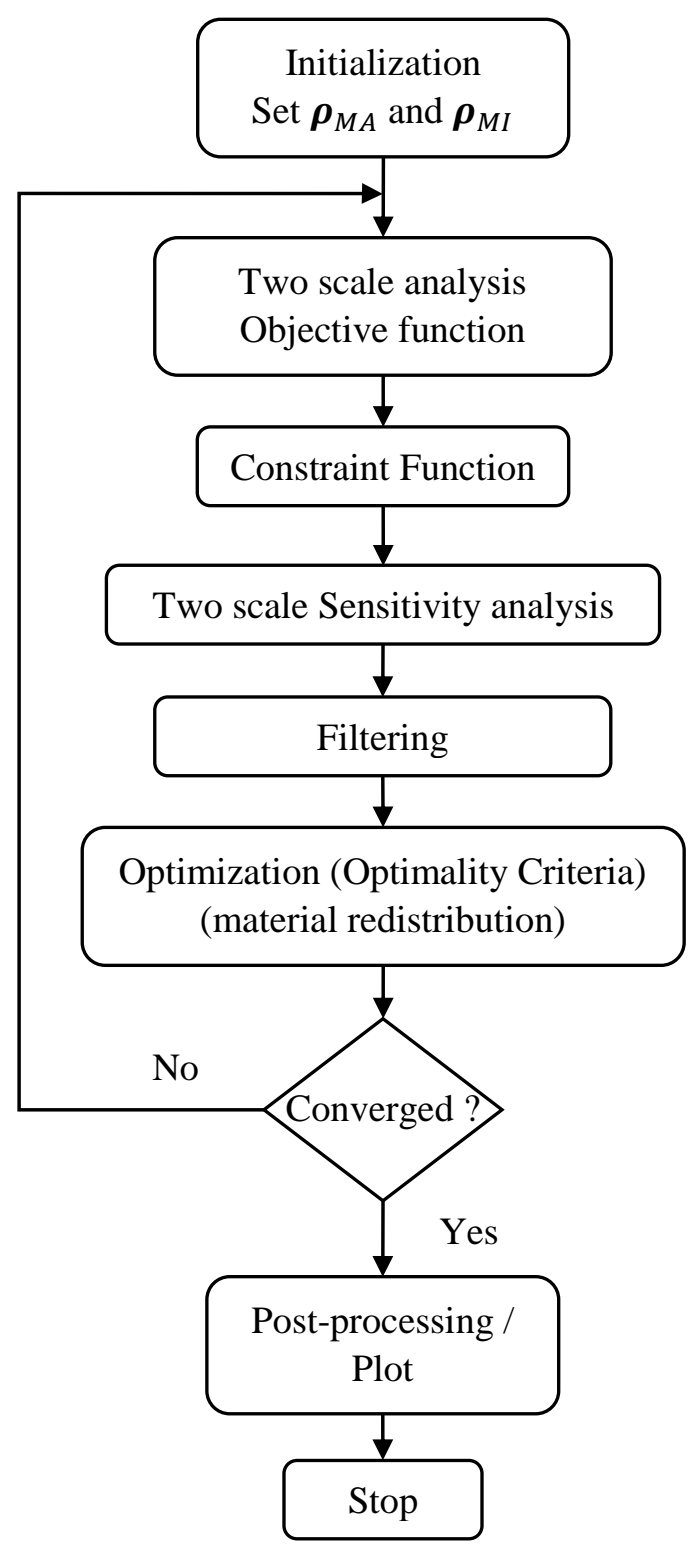

Figure 3-5 - Flowchart of the Porous Anisotropic Material with Penalization process.

\subsection{1}

\section{Sensitivity Analysis}

Rewriting the structural compliance from equation (4) in terms of the $\mathrm{N}$ element's compliance.

$$
c=\sum_{e=1}^{N} c_{e}=\sum_{e=1}^{N} \mathbf{F}_{e}^{T} \mathbf{U}_{e}
$$

Let $\boldsymbol{X}$ be the set with of all design variables and can be written as 


$$
\boldsymbol{X}=\left[\begin{array}{l}
\boldsymbol{\rho}_{M A} \\
\boldsymbol{\rho}_{M I}
\end{array}\right]
$$

using equations (21), (22), and (9) we have,

$$
\frac{\partial c}{\partial \boldsymbol{X}}=-\sum_{e=1}^{N} \mathbf{U}_{e}^{T} \frac{\partial \mathbf{K}_{e}}{\partial \boldsymbol{X}} \mathbf{U}_{e}
$$

\subsubsection{1}

\section{Macroscale}

To derivate, the sensitivity with respect to the macroscale variable we begin with equation (23), but this time the design variables are the density of the macroscale $\left(\rho_{M A}\right)_{i}$

$$
\frac{\partial c}{\partial\left(\rho_{M A}\right)_{i}}=-\sum_{e=1}^{N} \mathbf{U}_{e}^{T} \frac{\partial \mathbf{K}_{e}}{\partial\left(\rho_{M A}\right)_{i}} \mathbf{U}_{e}
$$

and replacing (20) in the equation (24) we have

$$
\frac{\partial c}{\partial\left(\rho_{M A}\right)_{i}}=-\sum_{e=1}^{N} \mathbf{U}_{e}^{T} \frac{\partial}{\partial\left(\rho_{M A}\right)_{i}}\left(\int_{\Omega_{e}} \mathbf{B}_{e}^{T} \mathbf{D}_{e}^{M A} \mathbf{B}_{e} d \mathrm{~V}\right) \mathbf{U}_{e}
$$

with (18) we have

$$
\begin{aligned}
& \frac{\partial c}{\left(\partial \rho_{M A}\right)_{i}} \\
& =-\sum_{e=1}^{N} \mathbf{U}_{e}^{T} \frac{\partial}{\partial\left(\rho_{M A}\right)_{i}}\left(\int_{\Omega_{e}} \mathbf{B}_{e}^{T}\left(\rho_{M A}\right)_{i}^{p_{M A}} \mathbf{D}_{e}^{H} \mathbf{B}_{e} d \mathrm{~V}\right) \mathbf{U}_{e}
\end{aligned}
$$

and doing the derivate and taking it out some terms of the integral

$$
\frac{\partial c}{\partial\left(\rho_{M A}\right)_{i}}=-p_{M A}\left(\rho_{M A}\right)_{i}^{p_{M A}-1} \mathbf{U}_{i}^{T} \int_{\Omega_{e}} \mathbf{B}_{e}^{T} \mathbf{D}_{e}^{H} \mathbf{B}_{e} d \mathrm{~V} \quad \mathbf{U}_{i}
$$




\subsubsection{2}

\section{Microscale}

To derivate, the sensitivity with respect to the microscale variable we begin with equation (23), but this time the design variables are the density of the microscale $\left(\rho_{M I}\right)_{j}$

$$
\frac{\partial c}{\partial\left(\rho_{M I}\right)_{j}}=-\sum_{e=1}^{N} \mathbf{U}_{e}^{T} \frac{\partial \mathbf{K}_{e}}{\partial\left(\rho_{M I}\right)_{j}} \mathbf{U}_{e}
$$

using the stiffness matrix from equation (20) in equation (28), we have

$$
\frac{\partial c}{\partial\left(\rho_{M I}\right)_{j}}=-\sum_{e=1}^{N} \mathbf{U}_{e}^{T} \frac{\partial}{\partial\left(\rho_{M I}\right)_{j}}\left(\int_{\Omega_{e}} \mathbf{B}_{e}^{T} \mathbf{D}_{e}^{M A} \mathbf{B}_{e} d \mathrm{~V}\right) \mathbf{U}_{e}
$$

and with equation (18), we have

$$
\begin{aligned}
& \frac{\partial c}{\partial\left(\rho_{M I}\right)_{j}} \\
& =-\sum_{e=1}^{N} \mathbf{U}_{e}^{T}\left(\int_{\Omega_{e}} \mathbf{B}_{e}^{T}\left(\rho_{M A}\right)_{e}^{p_{M A}} \frac{\partial \mathbf{D}_{e}^{H}}{\partial\left(\rho_{M I}\right)_{j}} \mathbf{B}_{e} d \mathrm{~V}\right) \mathbf{U}_{e}
\end{aligned}
$$

where the $\mathbf{D}^{H}$ and the derivative with respect to $\boldsymbol{\rho}_{M I}$ are shown in section 3.4.

\subsection{2}

\section{PAMP Multi-region}

The PAMP method can also be used to generate different design regions [91]. The process is similar to the Discrete Material Optimization method (DMO) [152] which was originally proposed to choose the fiber orientation in a set of composite materials and later was extended to topology optimization $[153,154]$. In the DMO, the elastic matrix $\mathbf{D}_{e}$ is denoted as the sum of the elastic matrix from different materials $\mathbf{D}_{\varpi}$ and his weight factor $x_{\varpi}$, as follows. 


$$
\mathbf{D}_{e}=\sum_{i=1}^{N_{\varpi}} x_{\varpi i}^{p_{\varpi}} \mathbf{D}_{\varpi i}
$$

where $N_{\varpi}$ is the number of candidate materials, $p_{\varpi}$ is the penalization factor, adopted from SIMP [17] to push towards 0 or 1 , and $x_{\varpi}$ are the design variables. The derivate with respect to $x_{\varpi}$ can be written as follows

$$
\frac{\partial \mathbf{D}_{e}}{\partial x_{\varpi}}=p_{\varpi} x_{\varpi}^{p_{\varpi}^{-1}} \mathbf{D}_{\varpi}
$$

so, the sensitivity analysis from equation (23) can be rewritten as follows

$$
\begin{aligned}
\frac{\partial c}{\partial x_{\varpi i}}=-\sum_{e=1}^{N} \mathbf{U}_{e}^{T} \frac{\partial \mathbf{K}_{e}}{\partial x_{\varpi i}} \mathbf{U}_{e} & \\
& =-\sum_{e=1}^{N} \mathbf{U}_{e}^{T} \frac{\partial}{\partial x_{\varpi i}}\left(\int_{\Omega_{e}} \mathbf{B}_{e}^{T} \mathbf{D}^{*} \mathbf{B}_{e} d V\right) \mathbf{U}_{e}
\end{aligned}
$$

with

$$
\mathbf{D}^{*}=\rho^{p} \mathbf{D}_{e}
$$

This method allows us to determine automatically the material (microstructure) for each subdomain. Figure 3-6 shows the topology optimization for a porous material at the macroscale with three different prescribed materials (red, green, blue). 

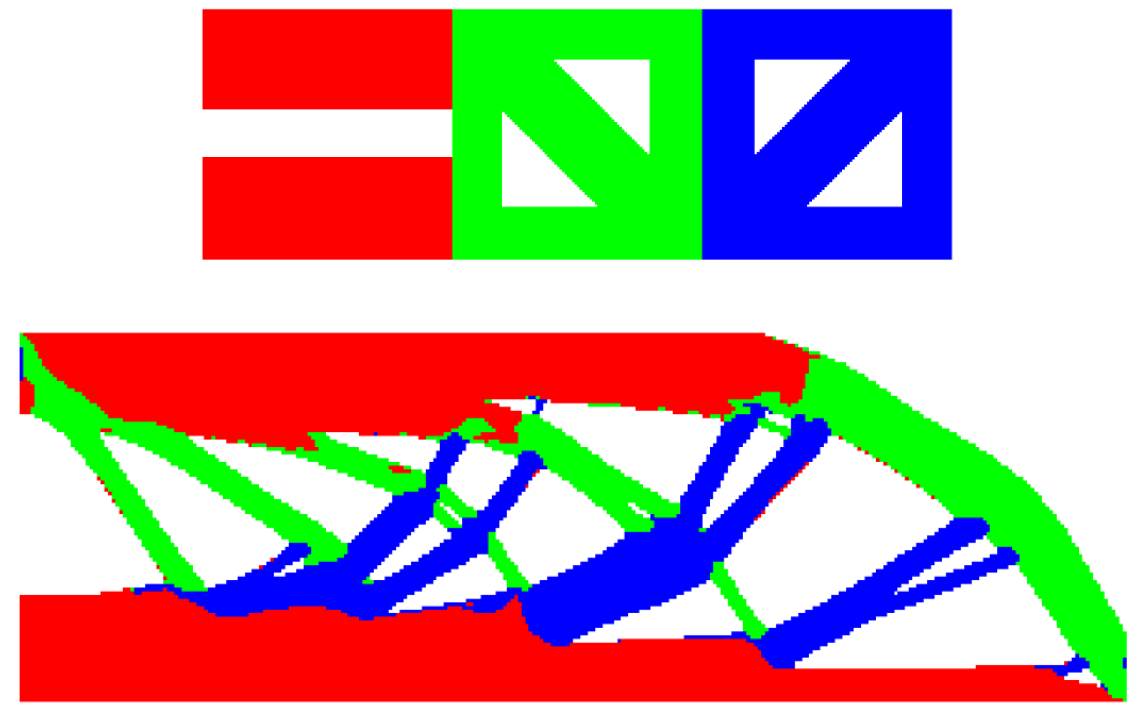

Figure 3-6 - Macrostructure optimized with DMO and composed of three different materials.

Figure 3-7 shows an arbitrary macrostructure composed of different porous material distributed by regions; these materials are made of a base material and can be of any type of solid material. As in the single region's PAMP [44], "two types" of material can be signalized in multi-region PAMP, the solid and porous material.

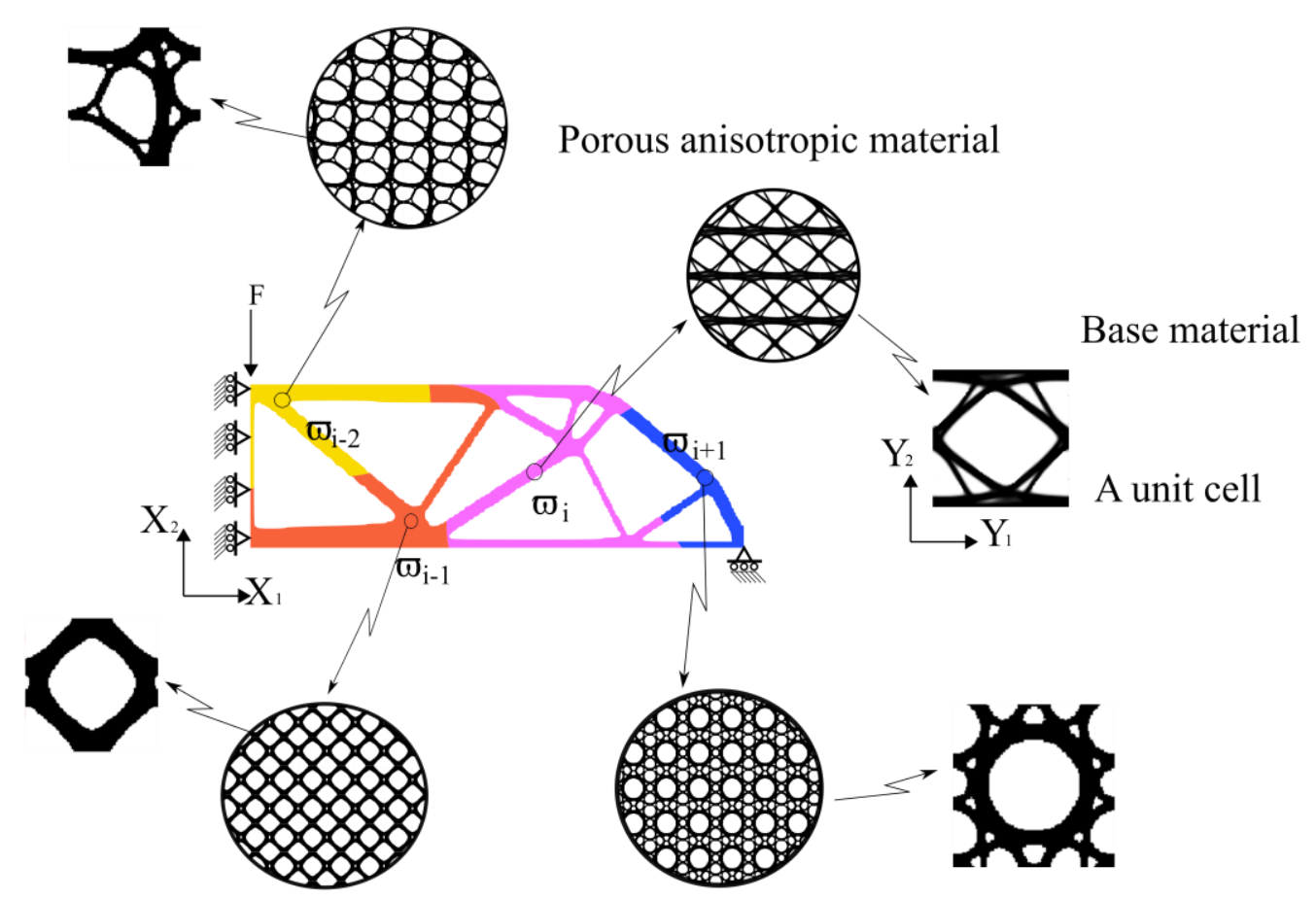

Figure 3-7 - An arbitrary Macrostructure composed of multiple porous regions. 
The mathematical formulation is similar to PAMP [44] but with a few small differences, As in PAMP two, independent variables are also defined i.e. the density at the macroscale $\rho_{M A}$ in the structural domain, and the density at the microscale $\rho_{M I}$. However, different from PAMP single-region, the number of micro densities is increased by each new material (region). The minimization problem shown in (16) can be rewritten as

$$
\begin{gathered}
\begin{array}{c}
\min \\
{\left[\boldsymbol{\rho}_{M A \varpi}, \boldsymbol{\rho}_{M I \varpi}\right]}
\end{array} \\
\text { subject to } \\
v=\sum_{i=1}^{N_{\varpi}} \frac{\left(\rho_{\varpi}^{P A M}\right)_{i} \int_{\Omega_{\varpi i}}\left(\boldsymbol{\rho}_{M A \varpi}\right)_{i} d \Omega_{\varpi i}}{A_{\varpi i}^{M A}} \leq \bar{v} \\
\left(\rho_{\varpi}^{P A M}\right)_{i}=\frac{\int_{\mathrm{Y}_{\varpi \mathrm{i}}}\left(\boldsymbol{\rho}_{M I \varpi}\right)_{i} d \mathrm{Y}_{\varpi \mathrm{i}}}{A_{\varpi i}^{M I}} \leq\left(\bar{v}_{\varpi}^{M I}\right)_{i} \\
0 \leq \boldsymbol{\rho}_{M A \varpi} \leq 1, \quad 0 \leq \boldsymbol{\rho}_{M I \varpi} \leq 1 \\
i=1, \ldots, N_{\varpi}
\end{gathered}
$$

where $\rho_{M A \varpi}$ and $\rho_{M I \varpi}$ are the density of macro and microscale for all elements of each region, $\varpi$ are the material's regions into which the problem is divided (different porous materials). $\left(\rho_{\varpi}^{P A M}\right)_{i}$ are the relative density of each porous materials that should be equals to the prescribed volume fraction of the material region $\left(v_{\varpi}^{M I}\right)_{i}$.

So, the equation (18) is rewritten using (31) as follows

$$
\left(\mathbf{D}_{\varpi}^{M A}\right)_{i}=\left(\rho_{M A \varpi}^{p_{M A}}\right)_{i} \mathbf{D}_{e}
$$

predefining the weighting factor of each subdomain in (32), i.e. $\frac{\partial \mathbf{D}_{e}}{\partial x_{\varpi}}=0$, leads a $\mathbf{D}^{H}$ to be a vector composed by $N_{\varpi}$ different subdomains and the new vector of design variables $\mathbf{X}$ of the equation (22) can be rewritten as follows

$$
\mathbf{X}=\left[\begin{array}{c}
\boldsymbol{\rho}_{M A} \\
\left(\boldsymbol{\rho}_{M I \varpi}\right)_{i}
\end{array}\right]=\left[\begin{array}{c}
\boldsymbol{\rho}_{M A} \\
\left(\boldsymbol{\rho}_{M I \varpi}\right)_{i} \\
\vdots \\
\left(\boldsymbol{\rho}_{M I \varpi}\right)_{n}
\end{array}\right]
$$


where $\boldsymbol{\rho}_{M A}$ is the set of design variables at the macroscale, and $\left(\boldsymbol{\rho}_{M I \varpi}\right)_{i}$ is the set of design variables at the microscale for the $i^{\text {th }}$ prescribed region.

\section{Sensitivity Analysis}

For the sensitivity analysis at the macroscale, the equation (27) is rewritten as follows

$$
\begin{aligned}
& \frac{\partial c}{\partial\left(\rho_{M A \varpi}\right)_{i}} \\
& =-\frac{p_{M A}}{\left(\rho_{M A \varpi}\right)_{i}} \mathbf{U}_{j}^{T}\left(\int_{\Omega} \mathbf{B}_{e}^{T}\left(\rho_{M A \varpi}^{p_{M A}}\right)_{i} \mathbf{D}_{e}^{H} \mathbf{B}_{e} d \Omega\right) \mathbf{U}_{j}
\end{aligned}
$$

and for the microscale, the equation (30) can be rewritten as

$$
\begin{aligned}
& \frac{\partial c}{\partial\left(\rho_{M I \varpi}\right)_{i, j}} \\
& =-\sum_{r=1}^{N_{\varpi}} \mathbf{U}_{r}^{T}\left(\int \mathbf{B}_{e} \rho_{M A r}^{p} \frac{\partial}{\partial\left(\rho_{M I \varpi}\right)_{i, j}}\left(\mathbf{D}_{\varpi}^{H}\right)_{i} \mathbf{B}_{e} d \Omega\right) \mathbf{U}_{r}
\end{aligned}
$$

where the $N_{\varpi}$ are the number of macro-elements in the each $\varpi$ region and $j$ are the design variables of each microstructure.

\section{4}

\section{Homogenization}

The equivalent properties of a microstructure can be obtained using the homogenization method [13]. According to [155] several methods have been proposed to obtain the equivalent properties of a microstructure such as the Surface average approach, volume average approach, force-based approach, Asymptotic Homogenization, among others. This process is widely used in different fields as piezoelectric actuators [156-163], biomedical applications [164-166], determine thermal and mechanical properties [167-170], and composite material in general.

Using the asymptotic homogenization [12, 13, 29, 30, 84], the homogenized stiffness tensor can be obtained by the average of the integral over the cell 


$$
\mathrm{D}_{i j k l}^{H}=\frac{1}{|Y|} \int_{Y} \mathrm{D}_{p q r s}\left(\varepsilon_{p q}^{0(i j)}-\varepsilon_{p q}^{*(k l)}\right) d Y
$$

where the $\varepsilon_{p q}^{0(i j)}$ is the two-dimensional or three-dimensional unit test strain fields and $\varepsilon_{p q}^{*(k l)}$ is the Y-periodic solution of the microscopic displacement [30]. According to $[32,171,172]$, the characterization of the equivalent behavior of composite materials can be done using an energy-based approach [173]. This approach employs the average stress and strain theorem over the entire cell $\mathrm{Y}$ in terms of the element mutual energies as

$$
\mathrm{D}_{i j k l}^{H}=\frac{1}{|Y|} \int_{Y} \mathrm{D}_{p q r s} \varepsilon_{p q}^{A(i j)} \varepsilon_{r s}^{A(k l)} d Y
$$

where $\varepsilon_{p q}^{A(i j)}$ is the superimposed strain field $\left(\varepsilon_{p q}^{0(i j)}-\varepsilon_{r s}^{*(k l)}\right)$ in equation (40), $|Y|$

is the area (or volume) of the cell, and $\mathrm{D}_{\text {pqrs }}$ is the $\mathbf{D}^{M I}$ used in (17). With a cell discretized into $N$ finite elements. Equation (41) can be rewritten for a finite element analysis as

$$
\mathrm{D}_{i j k l}^{H}=\frac{1}{|Y|} \sum_{e=1}^{N}\left(\mathbf{U}_{e}^{\mathrm{A}(i j)}\right)^{T} \mathbf{K}_{e} \mathbf{U}_{e}^{A(k l)}
$$

where $\mathbf{U}_{e}^{A(k l)}$ is the element displacement corresponding to the solution of the unit strain field test $\varepsilon_{e}^{A(k l)}$, and $\mathbf{K}_{e}$ is the element stiffness matrix. The equation (42) in the $2 \mathrm{D}$ case can be written in the expanded form as

$$
\mathbf{D}^{H}=\left[\begin{array}{lll}
\mathrm{D}_{11}^{H} & \mathrm{D}_{12}^{H} & \mathrm{D}_{13}^{H} \\
\mathrm{D}_{21}^{H} & \mathrm{D}_{22}^{H} & \mathrm{D}_{23}^{H} \\
\mathrm{D}_{31}^{H} & \mathrm{D}_{32}^{H} & \mathrm{D}_{33}^{H}
\end{array}\right]
$$

Figure 3-8 shows a base cell, before and after applying the unit test strain field. 


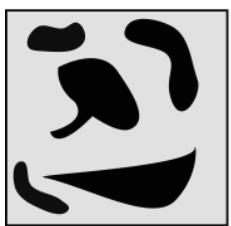

a)

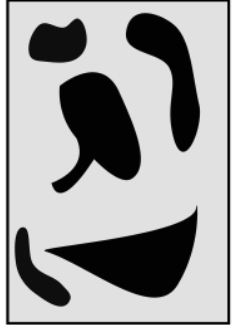

b)

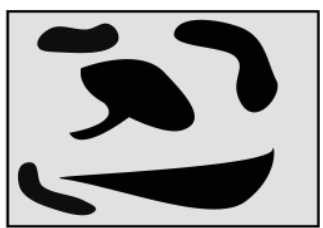

c)

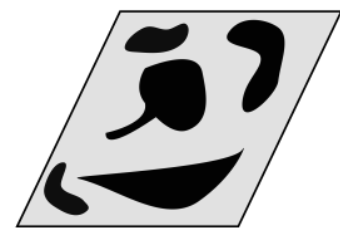

d)

Figure 3-8 - Periodic Boundary Conditions (PBC), a) Base cell. b) Deformation induced by unit initial strain in the vertical direction. c) Deformation induced by unit initial strain in the horizontal direction. d) Deformation induced by unit initial shear strain.

The sensitivity analysis of the equation (42) as a function of the design variables $[32,172,174]$ is

$$
\begin{aligned}
& \frac{\partial \mathrm{D}_{i j k l}^{H}}{\partial \rho_{M I}}= \\
& \quad \frac{1}{|Y|} p_{M I} \rho_{M I}^{p_{M I}-1}\left(E_{0}-E_{m i n}\right)\left(\mathbf{U}_{e}^{A(i j)}\right)^{T} \mathbf{K}_{0} \mathbf{U}_{e}^{A(k l)}
\end{aligned}
$$

where $\mathbf{K}_{0}$ is the element stiffness matrix for an element with unit Young's modulus.

\section{Periodic boundary conditions}

The $\varepsilon_{p q}^{A(i j)}$ from (41) are evaluate by solving the equilibrium problem subject to the unit test strain $\varepsilon_{p q}^{0(i j)}$. On the assumption of periodicity, the displacement of the cell subject to a given strain $\varepsilon_{i j}^{0}$ can be written as the sum of a macroscopic displacement and a periodic fluctuation field $u_{i}^{*}[31,172]$ as

$$
u_{i}=\varepsilon_{i j}^{0} y_{j}+u_{i}^{*}
$$

Since $u_{i}^{*}$ is unknown, equation (45) cannot be directly imposed on the boundaries. So, need to be described in explicit constraints between the corresponding pairs of nodes in the opposite surfaces of the cell. Figure 3-9 shows 
a cell in $2 \mathrm{D}$ at the microscopic scale where the $k-$ and $k+$ are the opposite parallel surfaces that are in perpendicular directions [175].

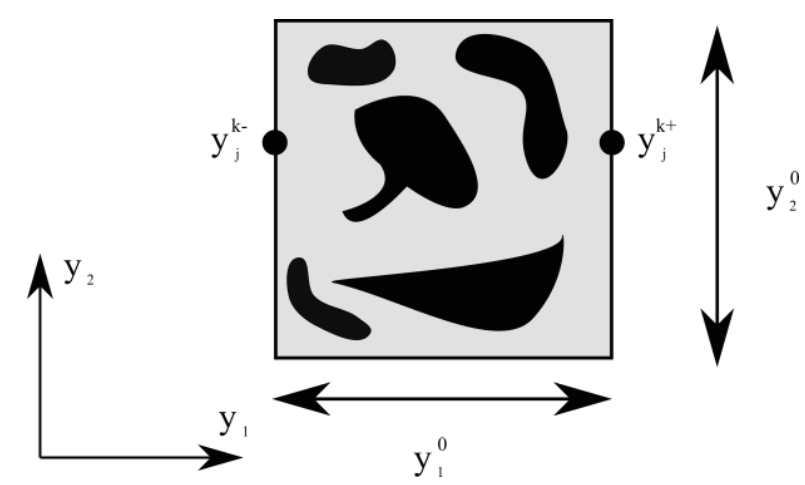

Figure 3-9 - Rectangular cell model in 2D adapted from [32].

So, the displacement of the pairs of nodes on the opposite boundary can be expressed as

$$
\left\{\begin{array}{l}
u_{i}^{k+}=\varepsilon_{i j}^{0} y_{j}^{k+}+u_{i}^{*} \\
u_{i}^{k-}=\varepsilon_{i j}^{0} y_{j}^{k-}+u_{i}^{*}
\end{array}\right.
$$

The periodic term $u_{i}^{*}$ in equation (46) can be eliminated through the difference between the displacements as follow

$$
u_{i}^{k+}-u_{i}^{k-}=\varepsilon_{i j}^{0}\left(y_{j}^{k+}-y_{j}^{k-}\right)=\varepsilon_{i j}^{0} \Delta y_{j}^{k}
$$

for a parallelepiped, the $\Delta y_{j}^{k}$ is constant with a specific $\varepsilon_{i j}^{0}$ and it can be directly imposed in the finite element model by constraining the corresponding pairs of nodal displacements [32].

\section{5}

\section{Connections Methods}

There are different methods to guarantee connectivity between cells, some of them have been compiled and tested in different works $[55,58,62]$. Here, will be introduced two of them. 


\subsection{1 \\ Kinematical Connective Constraint Method (KC)}

The method consists of prescribing connectors in the cell's edge that work to impose connective constraints. These connectors need to be permanently occupied by solid material, serving as imposed connective region to enforce the continuous edge-to-edge bonds between adjacent cells. The size, location, and number should be approximated to the periodic boundary conditions. It's worth pointing out that these connectors modify the solution and lead to different final topologies in the homogenization process [62]. Figure 3-10 shown the FGM (Functionally Graded Material) $[62,93]$ with an $\mathrm{N}$ number of cells with different volumes (Gradient Direction), and they are periodically repeated (periodic direction). The prescribed connectors in all the base cells are shown in red.

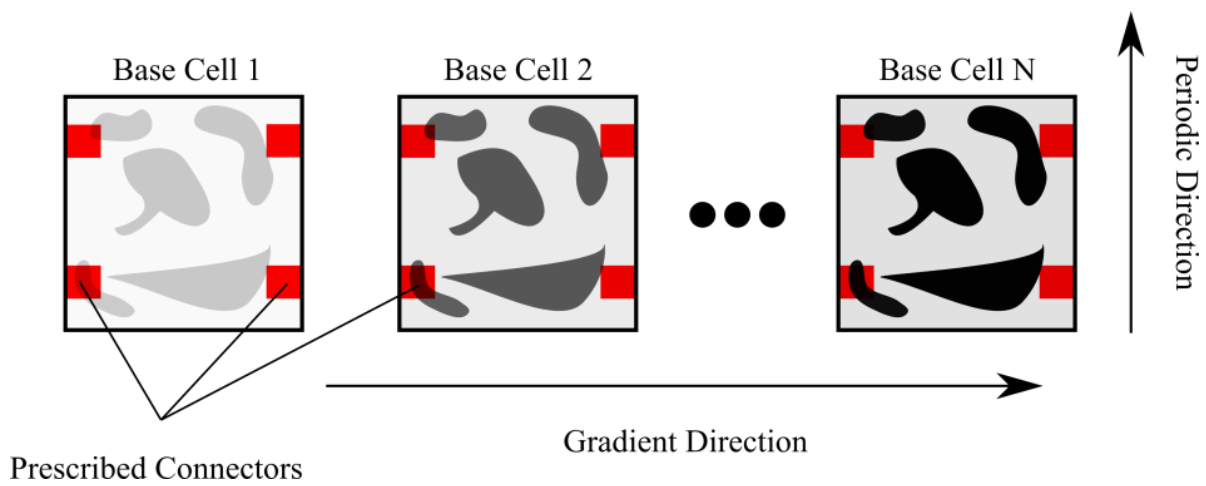

Figure 3-10 - Kinematical connector adapted from [62].

\subsection{2}

\section{Pseudo Load}

The main idea of the connectivity between cells is that they can transfer and sustain the imposed force through the whole body with a certain stiffness. Hence, a Pseudo Load (PL) can be applied, as shown in Figure 3-11, attempting to preserve the periodic condition like in the kinematical connector method [62]. 


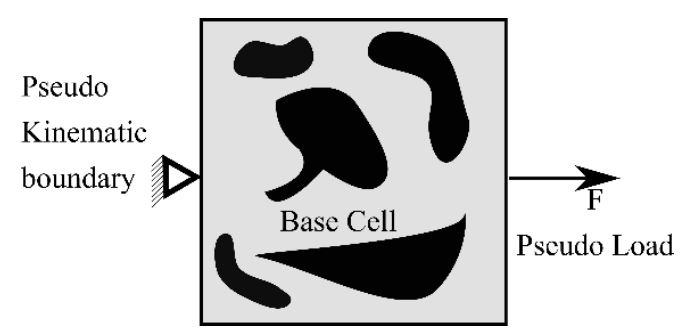

Figure 3-11 - Pseudo Load adapted from [62].

Then, through the application of the method, it is possible to include a pseudo stiffness criterion at the objective function, in a compliance term, as

$$
\begin{aligned}
\min j(\rho)= & \sum_{i j k l=1}^{3}\left(\dot{\mathbf{D}}_{i j k l}^{H}-\mathbf{D}_{i j k l}^{H}\right)^{2} \\
& +\eta \int_{\Omega} \boldsymbol{\varepsilon}_{L}\left(\mathbf{u}_{L}\right) \mathbf{D}(\rho) \boldsymbol{\varepsilon}_{L}\left(\mathbf{u}_{L}\right) d \Omega
\end{aligned}
$$

where the first term is the least-squares of the difference between the target $\dot{\mathbf{D}}_{i j k l}^{H}$ and the homogenized effective elastic tensor $\mathbf{D}_{i j k l}^{H}$ so-called inverse homogenization $[62,173]$. The strain and displacement induced by the PL are denoted as $\boldsymbol{\varepsilon}_{L}$ and $\mathbf{u}_{L}$, respectively, and $\eta$ is a weighting factor. To avoid overcontrol of the PL in the design process the second term needs to be small when compared with the first term of equation $(48)$, therefore, $\eta$ must have low values $(\approx$ 0.02). The method can be applied together with the KC method usually lead to proper connective zones. 


\section{4 \\ Transitional Microstructure for Smooth Connections Between Adjacent Regions (TrMIC)}

This chapter describes the proposed transitional microstructure for smooth connection between adjacent regions (TrMIC) method. The following sections will be discussed the motivation, the idea that inspired the scheme, and the mathematical formulation of the proposal. Also, will be explained how the proposed scheme is applied concurrently with the TO process. In addition, will be discussed the inherent problems of the method and how to address them. Finally, the advantages and disadvantages regard to the literature will be discussed.

\section{1}

\section{Motivation}

As discussed in the introduction of this thesis, the need for materials design emerged when the first composite materials appear, and all efforts were focused on the design of efficient structures (here every point in the structure should be designed for local conditions) through the combination of structural shape and microstructural material design [2, 26, 27].

In the design of hierarchical structures with different microstructures, where different parts or regions of the structure must be designed for specific needs, the lack of connectivity becomes a key limitation. In this context, topology optimization can give a solution where adjacent regions with different microstructures are not fully connected. The irregularities between microstructures can create stress concentration points and internal penetrations that can propagate cracks leading to failure on a large scale. Figure 4-1 illustrates the above-mentioned problem. Figure shows a topologically optimized solid with two different types of microstructures (1 and 2) and an enlarged area of the interface. As observed, the two microstructures are not fully connected on the edges (red circles). 


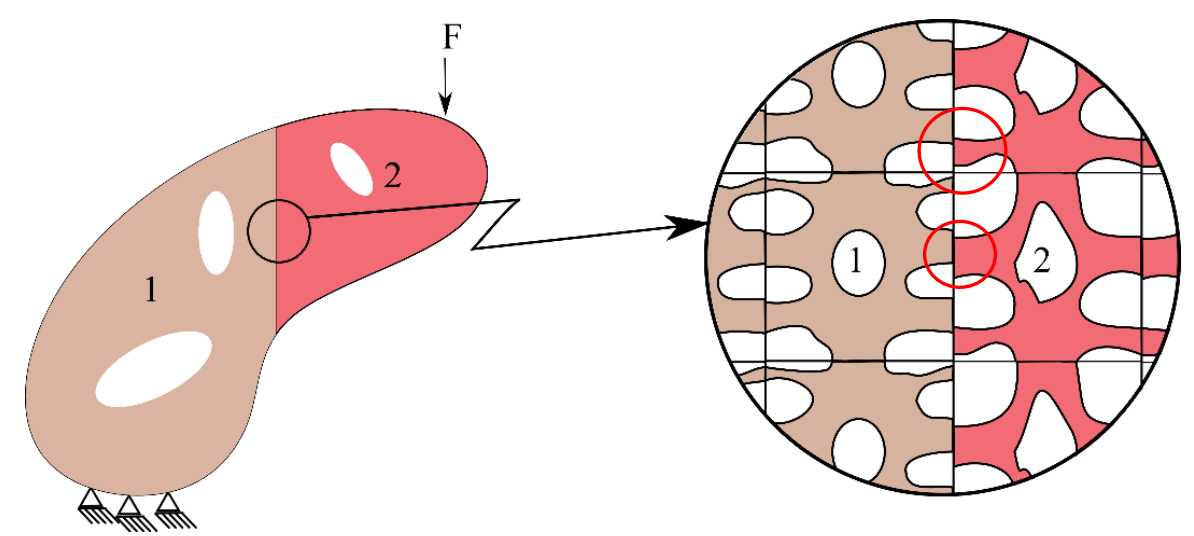

Figure 4-1 - Topologically optimized solid with two different types microstructures with discontinuities.

This thesis proposes a compatibility scheme for multiscale topology optimization by the introduction of a transitional microstructure, inspired by the role of the tendons tissue in the human body. The human body has about 200 different types of cells, with different sizes and shapes [176]. Cells jointly form different types of tissues that are the building blocks of organs. The skeletal muscle, the bones, and tendons are three different tissues that permit mobility. The bone provides structure and support, the skeletal muscle permits the movement of the body, and the tendons are the mechanism by which muscles connect to bone (see Figure 4-2) [177, 178]. In a simplified way, it could be said that the muscle and bone are the two principal microstructures in a hierarchical solid, and tendons are a transition microstructure that joins the first two.

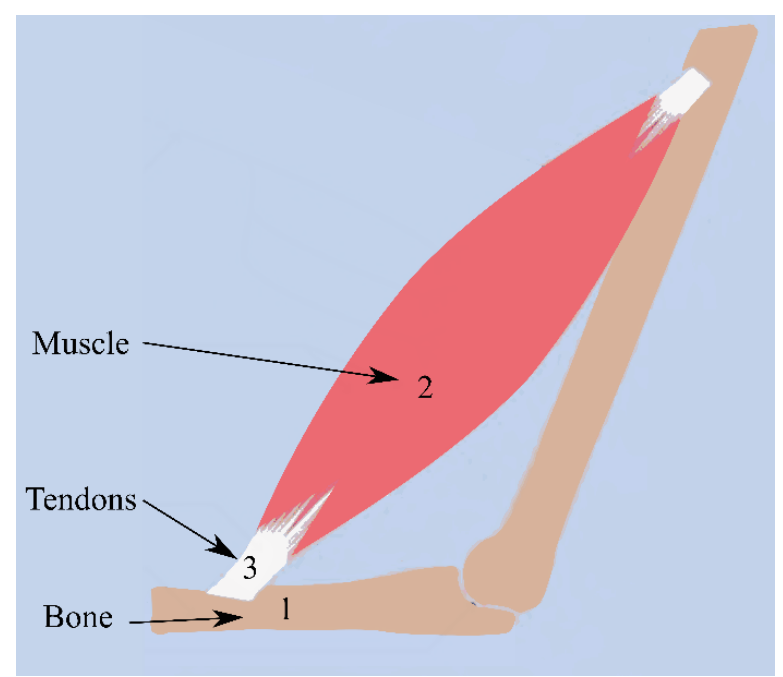

Figure 4-2 - Muscle, tendons, and bone [178]. 
Inspired by the above, this work proposes a Transitional MICrostructure (TrMIC) that uses the pseudo load (PL) and the kinematical constraint (KC) to perform the same function as tendons, bounding adjacent microstructures with different topologies.

To develop the scheme, the PAMP method [44] was used to design both the macro and microstructure for a single material, and in order to design a multimaterial solid, the PAMP method is combined with the DMO method [152]. The link between scales was achieved by employing an energy-based homogenization process, and applying periodic boundary conditions [172, 173]. To ensure de compatibility of the transition microstructure, a two-step Pseudo Load-based (PL) method in combination with kinematical constraints $(\mathrm{KC})$ is proposed, and this process is applied concurrently to the TO process.

Figure 4-3 shows a solid with three different types of microstructure, two of them are the principal regions, which represent the bone and the muscle (1 and 2), and the third in the middle is the TrMIC (3), which represents the tendons.

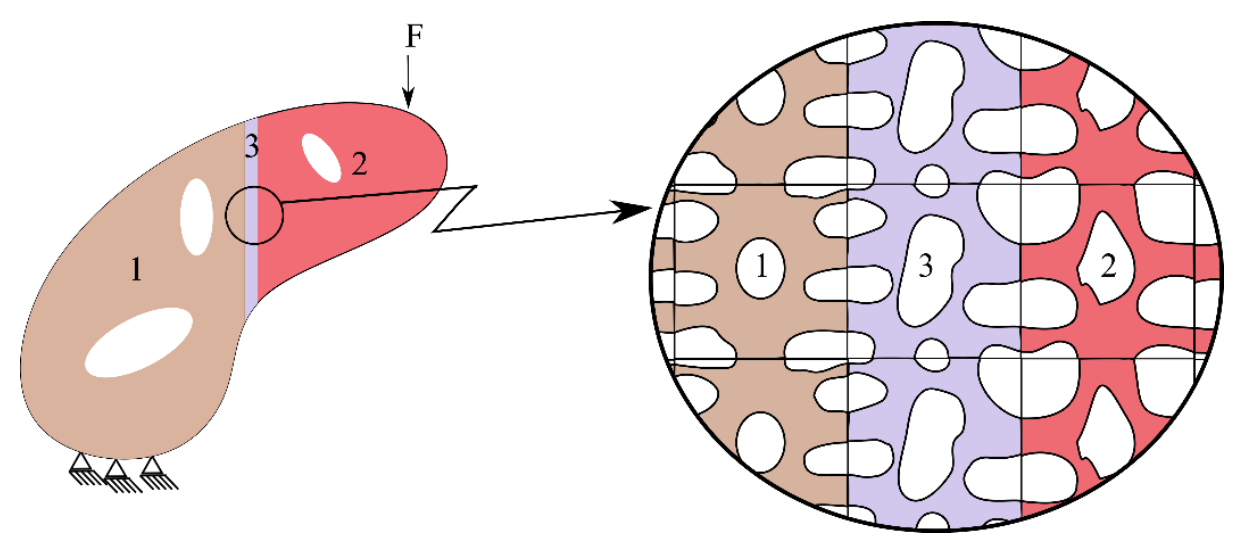

Figure 4-3 - Two cell (1 and 2) bonded by the TrMIC (3).

The next section will discuss the process necessary to obtain the solution illustrated in Figure 4-3, the mathematical formulation, and the steps needed to implement the proposed scheme concurrently with TO.

\section{2}

\section{Proposed Method}

Figure 4-4 a) shows two well-defined cells ( 1 and 2) that should be bonded by the TrMIC (3). The black regions in the cells' edges (1 and 2) are the points where 
the TrMIC needs to add the material for a better transition. To achieve the connectivity, PL and KC methods could be used (see section 3.5). In Figure 4-4 a) the black regions become the pseudo loads and kinematic boundaries. However, by using the PL and $\mathrm{KC}$ as originally formulated, undesired solutions may appear (Figure 4-4 b) and c)) since the transition region doesn't fit the periodic boundary conditions (PBC). Nonetheless, this study proposes an adaptation to the PL method to address this problem. The proposed modification consist in a two-step PL-based method that combines the two-pseudo loads cases present in TrMIC.

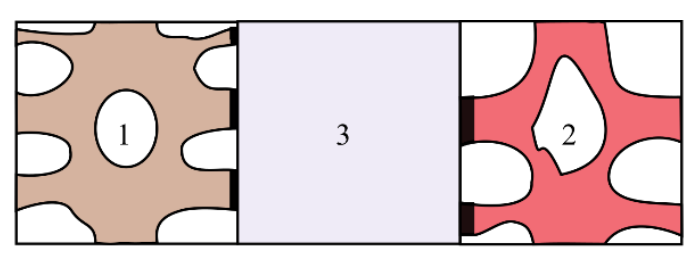

a)

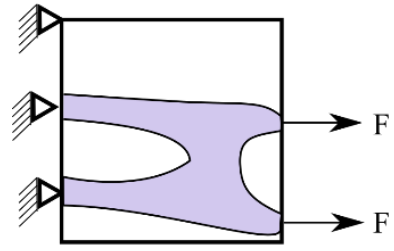

b)

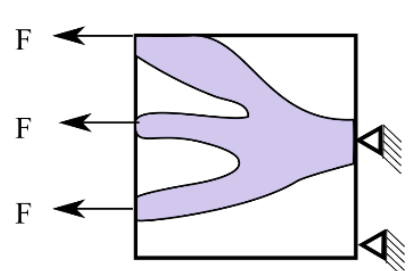

c)

Figure 4-4 - a) TrMIC under non-PCB. b) Case 1: load on the right (fixed-left) and c) Case 2: load on the left (fixed right).

Using equations (42) and (48); the following equation is proposed

$$
\begin{aligned}
& \min j(\rho)=\frac{1}{|Y|} \sum_{e=1}^{N}\left(\mathbf{U}_{e}^{\mathrm{A}(i j)}\right)^{T} \mathbf{K}_{e} \mathbf{U}_{e}^{A(k l)} \\
&+\eta_{\lambda} \int_{\Omega} \boldsymbol{\varepsilon}_{L \lambda}\left(\mathbf{u}_{L \lambda}\right) \mathbf{D}(\rho) \boldsymbol{\varepsilon}_{L \lambda}\left(\mathbf{u}_{L \lambda}\right) d \Omega
\end{aligned}
$$

where $\boldsymbol{\varepsilon}_{L \lambda} \cdot \mathbf{u}_{L \lambda}$ and $\eta_{\lambda}$ with $\lambda=1,2$ Are the strain, displacement and weighting factor for both PL cases.

The sensitivity analysis of equation (49) as a function of the design variables is given by 


$$
\begin{aligned}
& \frac{\partial \mathbf{D}_{i j k l}^{H}}{\partial \rho_{M I}}=-\frac{1}{|Y|} p_{M I} \rho_{M I}^{p_{M I}-1}\left(E_{0}-E_{\min }\right) \\
& \left(\left(\mathbf{U}_{e}^{A(i j)}\right)^{T} \mathbf{K}_{0} \mathbf{U}_{e}^{A(k l)}+\sum_{\lambda=1}^{2} \eta_{\lambda}\left(\mathbf{U}_{e}^{L \lambda}\right)^{T} \mathbf{K}_{0} \mathbf{U}_{e}^{L \lambda}\right)
\end{aligned}
$$

The two-step PL and the KC must be added concurrently to the PAMP TO multiregion, as shown in Figure 4-5. The process is gradual and must be applied as soon as the boundary elements reach a density value at least $1 \%$ greater than the initial guess value in the optimization process (represented by the black regions). Figure 4-5 a) shows the first iterations of the optimization process, where the first black regions (i.e. the connection regions) emerge in the principal cells (1 and 2), and each step of the two-step PL and KC are applied. Figure 4-5 b) shows an iteration at some point in the middle of the optimization process. Here more black regions emerge, and more pseudo loads need to be added. Finally, Figure 4-5 c) shows the final topology for all cells. Notice that the TrMIC is now fully connected.

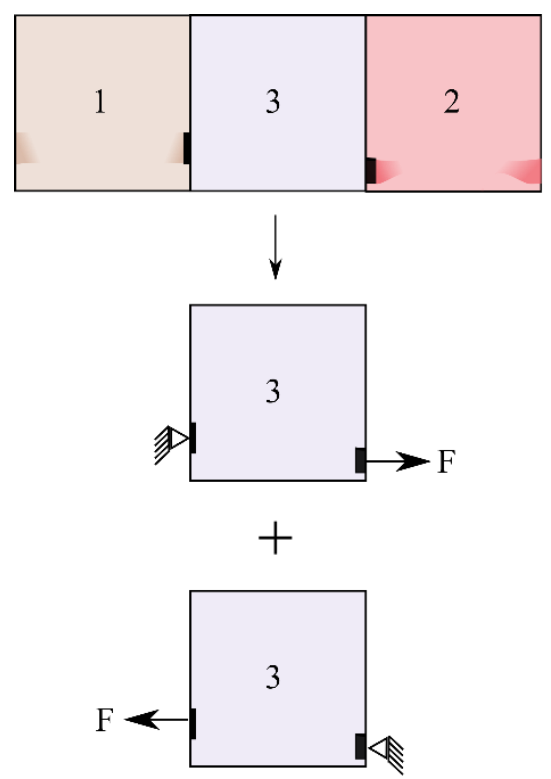

a)

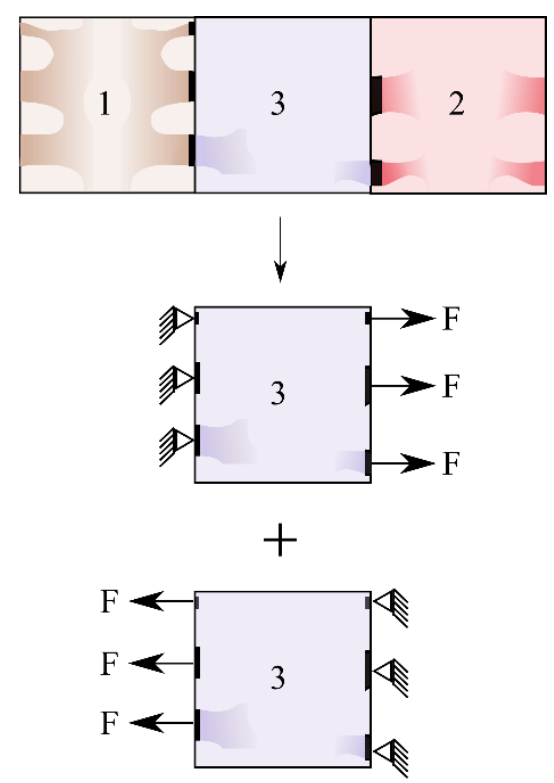

b)

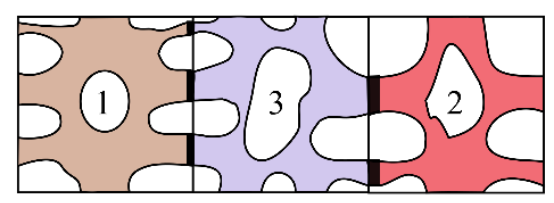

c) 
The weight factors $\eta_{\lambda}$ in equation (50), are proposed to calculated as the ratio between the magnitude of the pseudo load and the homogenization sensitivities. Therefore, $\eta_{\lambda}$ can be expressed as

$$
\eta_{\lambda}=\frac{\max (\mathbf{A})}{\max (\mathbf{B})}
$$

where $\mathbf{A}$ is the first part of the equation (50)

$$
\mathbf{A}=\left(\mathbf{U}_{e}^{A(i j)}\right)^{T} \mathbf{K}_{0} \mathbf{U}_{e}^{A(k l)}
$$

and $\mathbf{B}$ is the second part of the equation (50)

$$
\mathbf{B}=\left(\mathbf{U}_{e}^{L \lambda}\right)^{T} \mathbf{K}_{0} \mathbf{U}_{e}^{L \lambda}
$$

Equation (51) allows being dynamically calculated the weight factor throughout the optimization process, leaving both parts in the same order preventing the dominance of either.

\section{3}

\section{Final Considerations and Discussion}

One drawback of the proposed scheme is the additional computational effort that is added. Every interface needs a TrMIC that must be homogenized, increasing the computational time to process the TO. Another problem that arises when modifying the final shape of the cell to adapt to connectivity is the loss in stiffness.

In contrast, compared with other methods [61, 62], the proposed scheme does not need predefined points to fit the connectivity because these are self-defined during the optimization process. Furthermore, different from other methods [58, 62], the proposed scheme does not require a tuning process for the weighting factor since it is calculated on the fly. Finally, the proposed scheme could potentially connect microstructures with different volume fractions, a problem that can be observed in $[61,63,64]$ and was discussed at the end of section 2.1.2.2. 


\section{5 \\ Numerical Examples}

This chapter presents the verification of the homogenization implementation also is present numerical examples for the PAMP method for single and multiscale cases, and the proposed approach. In addition, a comparison with different examples in literature is performed in order to evaluate the compatibility scheme. Finally, 3D printed samples and the ANSYS analysis for the MBB-beam problem [179] for classic TO and the Variable-density are presented and analyzed.

\section{1}

\section{Evaluating the implemented codes}

This section presents the verification of the implementation of the homogenization and the PAMP method. To implement these examples was used a MATLAB [180] code based on previous research, the 88 lines code [143], and the topX code $[32,172]$.

\subsection{1 \\ Homogenization}

To verify the code implementation, the solution was compared with the example presented by Wang et.al [90]. Figure 5-1 shows the comparison for two different microstructures (left), their homogenized tensor (middle), and the result obtained in this study (right). The experiments were carried out using a Young's module $E=$ $1000 \mathrm{MPa}$, a Poisson's ratio $v=0.3$, and a mesh with $100 \times 100$ elements. As observed, both implementations presents similar results. Since there is not enough information to design a cell that match perfectly those reported in [90], we construct cells as close as possible keeping the width of the horizontal and vertical branches. This explain the small difference in the values. 


\begin{tabular}{|c|c|c|c|c|c|c|}
\hline microstructure & \multicolumn{3}{|c|}{$\begin{array}{l}\text { Homogenized tensor from } \\
\qquad[90]\end{array}$} & \multicolumn{3}{|c|}{$\begin{array}{l}\text { Homogenized tensor with } \\
\text { the implemented code }\end{array}$} \\
\hline & {$[236.29$} & 107.57 & 0 & {$[234.28$} & 112.53 & 0 \\
\hline & 107.57 & 236.29 & 0 & 112.53 & 234.28 & 0 \\
\hline & 0 & 0 & 101.99 & 0 & 0 & 105.50 \\
\hline & {$[319.62$} & 36.24 & 0 & Г320.38 & 36.33 & 0 \\
\hline & 36.24 & 319.62 & 0 & 36.33 & 320.38 & 0 \\
\hline & 0 & 0 & 15.30 & 0 & 0 & 14.36 \\
\hline
\end{tabular}

Figure 5-1 - Comparison between homogenized tensors.

\section{1 .2}

\section{Single Region MBB-beam Problem}

In order to compare the implementation of the PAMP method, different tests were conducted and compared with other solutions reported in the literature. Figure 5-2 shows an MBB-beam [179, 181] problem, with height (h), a length (L), and a force $(F)$. Due to the symmetry of the problem the experiment was conducted only in one half (the gray one) at the macroscale. Also, on the right side is pointed out the micro design domain of which is composes the macroscale.

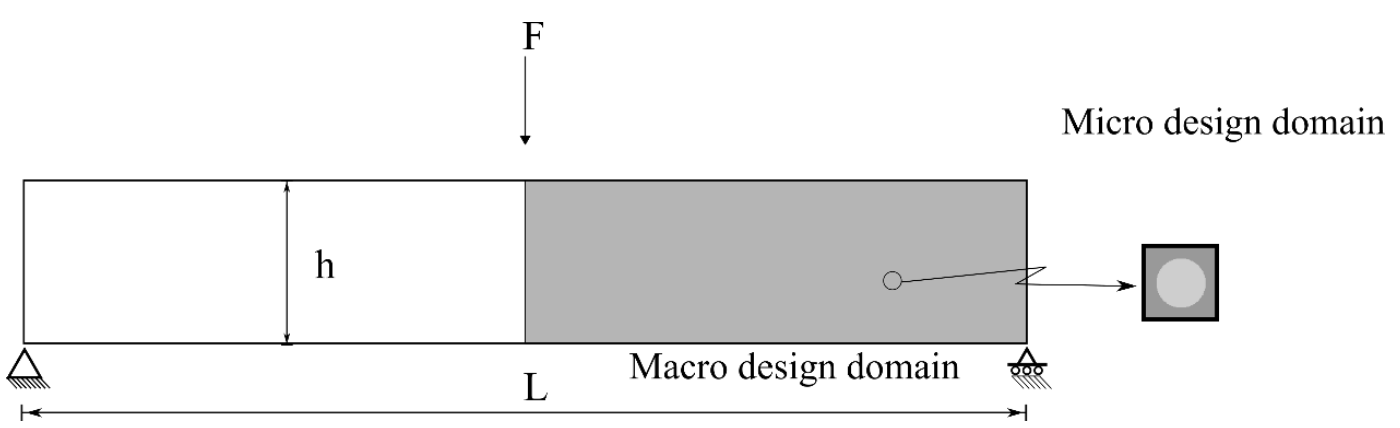

Figure 5-2 - MBB-beam example (macro and micro design) adapted from [44].

Figure 5-3 shows the solution using the ConTop2D code [84] for different values of volume at the macroscale (first column) with a fixed volume for the microscale domain (second column), the value of resulting compliance using the homogenized stiffness tensor. (third column), the final topology for the macroscale (fourth column), and the microscale final topology (fifth column) are presented. The discretization of the macroscale is $50 \times 25$ elements and $100 \times 100$ elements for the microscale. A density filter is applied with radius at the macroscale $r$ min $=2$ 
(in element`s number) and a penalization factor $p=3$. The length $L=4$, and the height $h=1$, the base material has Young's modulus $E=2.5 \times 10^{5}$,and Poisson's ratio $v=0.3$.

\begin{tabular}{ccccc}
\hline volfrac $_{M A}$ & volfrac $_{M I}$ & $\begin{array}{c}\text { Compliance } \\
\text { (c) }\end{array}$ & $\begin{array}{c}\text { Macrostructural } \\
\text { Topology }\end{array}$ & $\begin{array}{c}\text { Microstructural } \\
\text { Topology }\end{array}$ \\
0.075 & 0.40 & 1227 & \\
0.12 & 0.40 & 610 & & \\
0.18 & 0.40 & 261 &
\end{tabular}

Figure 5-3 - MBB-beam using multiscale composite [84].

Figure 5-4 shown the result of the PAMP using our implementation. In our test was used a density filter with radius at the macroscale $\operatorname{rmin}_{M A}=1.2$ (in element`s number), at the microscale $\operatorname{rmin}_{M I}=3$ (in element's number), also was used a penalization factor at the macroscale $p_{M A}=1-3$, and for the microscale $p_{M I}=4$, the cell initialization is the same used in [172]. This was the best parameter configuration found in a preliminary test. 


\begin{tabular}{ccccc}
\hline volfrac $_{M A}$ & volfrac $_{M I}$ & $\begin{array}{c}\text { Compliance } \\
\text { (c) }\end{array}$ & $\begin{array}{c}\text { Macrostructural } \\
\text { Topology }\end{array}$ & $\begin{array}{c}\text { Microstructural } \\
\text { Topology }\end{array}$ \\
0.075 & 0.40 & 1284 & \\
0.12 & 0.40 & 860 & & \\
0.18 & 0.40 & 064 &
\end{tabular}

Figure 5-4 - Results of PAMP MBB-beam 1 cell.

The macro-structural topology, as expected, has considerable changes when more base material is added, and therefore, better stiffness is achieved. The microstructure for both, Figure 5-3 and Figure 5-4, does not have visual significant changes (similar stiffness tensor), which is normal behavior since all the macrostructural elements are subjected to the same stress distribution in all tests.

\section{1 .3}

\section{Single Region L-shape Problem}

The second test used a problem with an L-shape domain [182], as shown in Figure 5-5, where the dimensions are $\mathrm{A}$ and $B=\lambda A$. $\mathrm{F}$ is a distributed force on the right edge. 


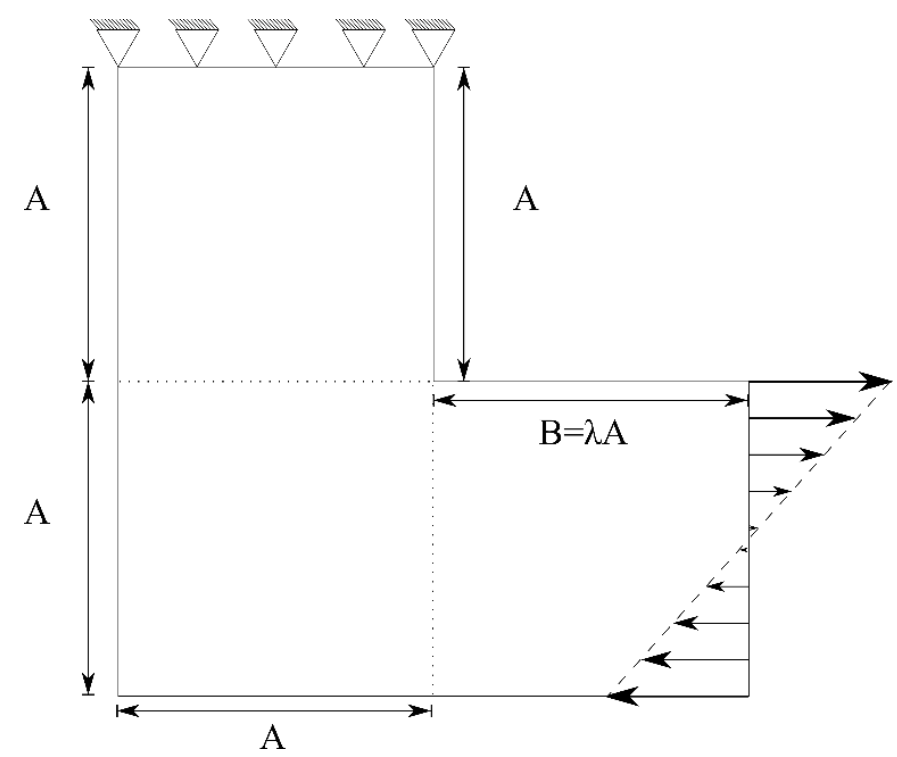

Figure 5-5 - L-shape problem 1.

Figure 5-6 shows the solution using the ConTop2D code [84] for a fixed volume fraction at the macroscale (first column), a fixed volume fraction at the microscale (second column), and a variable multiplier value $\lambda$ (third column). The ratio between de $\mathrm{x}$ and y components of Young's module (fourth column). Finally, the structural topology at macro and microscale (fifth - last column). For this experiment A was setting equals 40 (elements).

\begin{tabular}{cccccc}
\hline volfrac $_{M A}$ & volfrac $_{M I}$ & $\lambda$ & $\mathrm{D}^{11} / \mathrm{D}^{22}$ & $\begin{array}{c}\text { Macrostructural } \\
\text { Topology }\end{array}$ \\
\hline 0.1 & 0.40 & 0 & 0.653 & $\begin{array}{c}\text { Microstructural } \\
\text { Topology }\end{array}$ \\
0.1 & 0.40 & 1 & 1.079 \\
0.1 & 0.40 & 2 & 1.357
\end{tabular}

Figure 5-6 - L-shape using multiscale composite [84].

Figure 5-7 shows our results for the L-shape shown in Figure 5-5 using the PAMP method. 


\begin{tabular}{llllll}
\hline volfrac $_{M A}$ & volfrac $_{M I}$ & $\lambda$ & $\mathrm{D}^{11} / \mathrm{D}^{22}$ & $\begin{array}{c}\text { Macrostructural } \\
\text { Topology }\end{array}$ \\
\hline 0.1 & 0.40 & 0 & 0.616 \\
0.1 & 0.40 & 1 & 1.066 \\
0.1 & 0.40 & 2 & 1.235
\end{tabular}

Figure 5-7 - Results and comparison of L-shape problem.

As shown in Figure 5-6 and Figure 5-7, the modulus ratio $\mathrm{D}^{11} / \mathrm{D}^{22}$ varies because the material is arranged to strengthen the porous material in the $\mathrm{x}$-direction as $\lambda$ varies. With $\lambda=0$ bar $A$ is dominant and this is reflected in the modulus ratio with the $\mathrm{D}^{22}$ (vertical) $60 \%$ higher, as shown in Figure 5-7. On the other hand, when $\lambda=3$, the "lever" has a major influence and $\mathrm{D}^{11}$ (horizontal) reaches its maximum value.

To better understand this behavior, the L-shape beam problem was divided into 3 regions considering the internal stress conditions (see Figure 5-8). In region \#1, the moments are imposed in the upper and lower faces, and the principal deformation is in the vertical direction. In region \#3 the moments are imposed on the left and right faces, and the principal deformation is in the horizontal direction. These two conditions are contradictory for the optimization of a single cell which cause the above mention behavior and therefore, a better solution could be a multiregion (multi-cell) approach. 


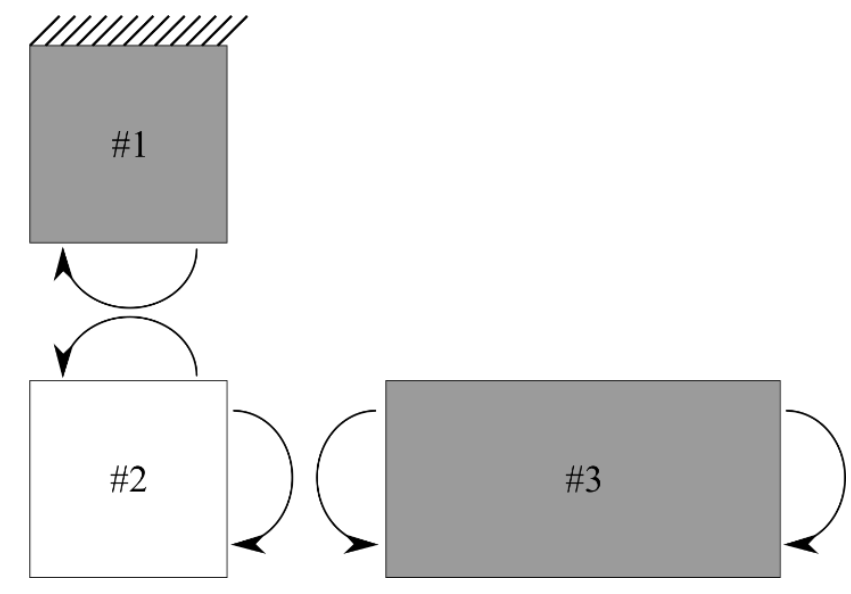

Figure 5-8 - Decomposition of the L-shape beam [44].

\section{2}

\section{Experiments for PAMP}

This section presents the verification of the homogenization and the PAMP method single region for the MBB-beam and the L-shape problems. Next, numerical examples of PAMP with different regions are presented. In addition, examples of the TrMIC scheme and a comparison with other connections methods are given. The values related to the mbb-beam problem were chosen in such a way that the solution obtained can be manufactured in a 3D printer and will be shown in section 5.3.

\subsection{1}

Experiments for the MBB-beam Problem

\section{Test 1: Single Region Solution}

Figure 5-9 shows the PAMP single region solution for the MBB-beam problem shown in Figure 5-2. The parameters configuration consist of $L=270$ and $h=90$ (in element's number), cells with $100 \times 100$ elements, a volume fraction at macroscale of $\operatorname{volfrac}_{M A}=0.42$, a volume fraction at microscale volfrac $_{M I}=$ 0.6 , Young's modulus $E=5.1 \times 10^{6}$, a force $F=1000$, radius at the macroscale $\operatorname{rmin}_{M A}=9$ elements, radius at the microscale of $\operatorname{rmin}_{M I}=5$ elements, and a penalization factor for the macrostructure $p_{M A}=3$ and for the micro $p_{M I}=4$. With 
this configuration and using the homogenized stiffness tensor the TO achieved an objective function value equals to 2299 .

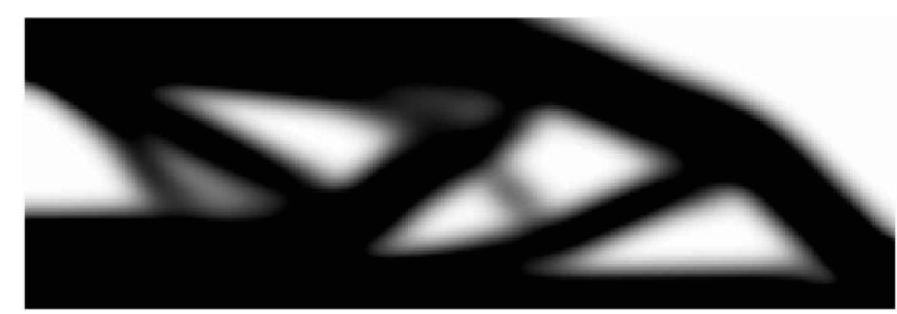

\begin{tabular}{|c|ccc|}
\hline Cell & \multicolumn{3}{|c|}{$\mathbf{D}^{H}$} \\
\hline & {$\left[\begin{array}{ccc}0.4513 & 0.0684 & -0.0387 \\
0.0684 & 0.1509 & -0.0274 \\
-0.0387 & -0.0274 & 0.0749\end{array}\right]$} \\
\hline
\end{tabular}

Figure 5-9 - Half MBB-beam solution using PAMP with 1 cell.

\section{Test 2: Multi-Region Solution for 3 Regions}

Figure 5-10 shows the implementation of PAMP multi-region for 3 different cells. The final macro (Figure 5-10 a) and microscale topology and their homogenized tensor (Figure 5-10 b) are presented. The three regions are composed of $30 \times 270$ elements. As pointed out by the orange ellipse (Figure 5-10 b) there are different regions where did not exists a connection between cells or the connection is poor. Using the homogenized stiffness tensor the TO achieved an objective function value equals to 2172 . 


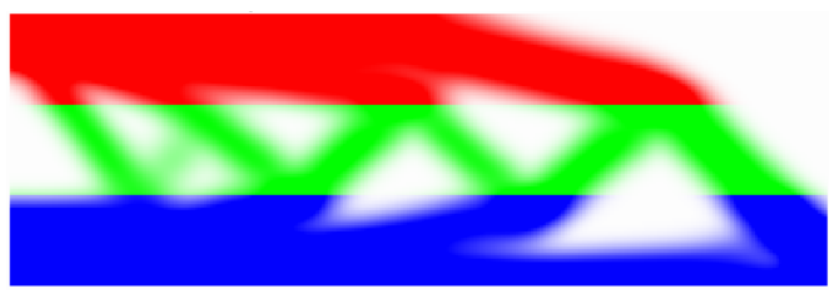

a)

\begin{tabular}{|c|c|c|c|}
\hline Cell & \multicolumn{3}{|c|}{$\mathbf{D}^{H}$} \\
\hline & {$[0.4653$} & 0.0562 & -0.0599 \\
\hline & 0.0562 & 0.1300 & -0.0397 \\
\hline & -0.0599 & -0.0397 & 0.0670 \\
\hline & {$[0.2636$} & 0.1731 & -0.0213 \\
\hline & 0.1731 & 0.2472 & -0.0226 \\
\hline & -0.0213 & -0.0226 & 0.1554 \\
\hline & {$[0.4553$} & 0.0599 & $-0.0025^{-}$ \\
\hline & 0.0599 & 0.1801 & -0.0174 \\
\hline & -0.0025 & -0.0174 & 0.0594 \\
\hline
\end{tabular}

b)

Figure 5-10 - Half MBB-beam solution using PAMP with 3 cells, a) macrostructure, b) microstructure, and homogenized stiffness tensor. The orange ellipse denotes the regions where did not exists a connection between cells or the connection is poor.

\section{Test 3: Multi-Region Solution for 5 Regions}

Figure 5-11 shows the solution of the PAMP multi-region for 5 different cells. The final macro (Figure 5-11 a) and microscale topology and their homogenized tensor (Figure 5-11 b) are presented. The number of elements of each region are the following: $20 \times 270$ for region one (red), $10 \times 270$ for region two (yellow), $30 \times$ 270 for region three (green), $10 \times 270$ for region four (blue), and $30 \times 270$ for region five (purple). As pointed out by the red circles (Figure 5-11 b) there are different regions where did not exists a connection between cells or the connection is poor. Using the homogenized stiffness tensor the TO achieved an objective function value equals to 2043 . 


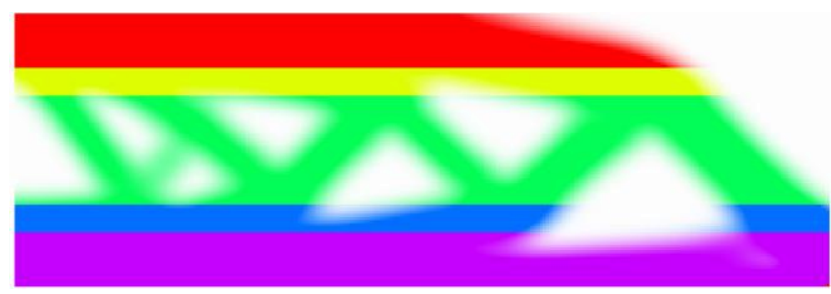

a)

\begin{tabular}{|c|ccc|}
\hline Cell & \multicolumn{3}{|c|}{$\mathbf{D}^{H}$} \\
\hline & {$\left[\begin{array}{llr}0.4768 & 0.0532 & -0.0507 \\
0.0532 & 0.1344 & -0.0427 \\
-0.0507 & -0.0427 & 0.0624\end{array}\right]$} \\
\hline \hline & {$\left[\begin{array}{llr}0.4407 & 0.0602 & -0.0825 \\
0.0602 & 0.1221 & -0.0249 \\
-0.0825 & -0.0249 & 0.0674\end{array}\right]$} \\
\hline & {$\left[\begin{array}{llr}0.2642 & 0.1706 & -0.0206 \\
0.1706 & 0.2499 & -0.0205 \\
-0.0206 & -0.0205 & 0.1542\end{array}\right]$} \\
\hline & {$\left[\begin{array}{lll}0.4350 & 0.0712 & 0.0266 \\
0.0712 & 0.1139 & -0.0244 \\
0.0266 & -0.0244 & 0.0797\end{array}\right]$} \\
\hline & {$\left[\begin{array}{lll}0.4669 & 0.0503 & -0.0067 \\
0.0503 & 0.1967 & -0.0311 \\
-0.0067 & -0.0311 & 0.0428\end{array}\right]$} \\
\hline
\end{tabular}

b)

Figure 5-11 - Half MBB-beam solution using PAMP TO with 5 cells, a) macrostructure, b) microstructure, and homogenized stiffness tensor. The red ellipse denotes the regions where did not exists a connection between cells or the connection is poor.

\section{Test 4: Multi-Region for 5 Cells using TrMIC}

Figure 5-12 shows the solution of the PAMP multi-region for 5 cells using TrMIC. The final macro (Figure 5-12 a) and microscale topology and their homogenized tensor (Figure 5-12 b) are presented. The five regions have the same number of elements as in Test 3. Region two (yellow) and four (blue) are the transition regions. As observed the connection between the cells red-yellow-green and green-blue-purple is qualitatively better in comparison with the presented in 
Figure 5-11. Using the homogenized stiffness tensor the TO achieved an objective function value equals to 2106 .

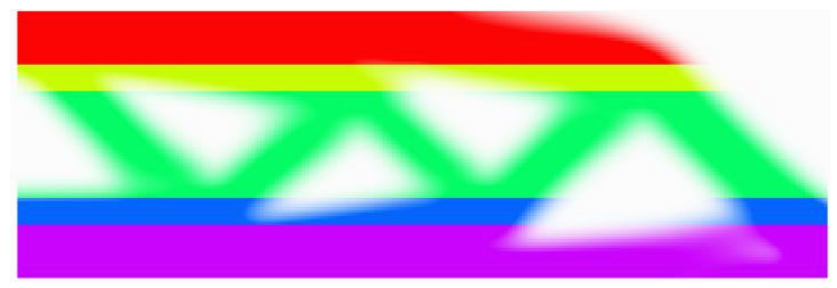

a)

\begin{tabular}{|c|c|c|c|}
\hline \multirow[t]{16}{*}{ Cell } & \multicolumn{3}{|c|}{$\mathbf{D}^{H}$} \\
\hline & {$[0.4812$} & 0.0516 & -0.0480 \\
\hline & 0.0516 & 0.1326 & -0.0421 \\
\hline & -0.0480 & -0.0421 & 0.0603 \\
\hline & {$[0.3536$} & 0.0808 & -0.0708 \\
\hline & 0.0808 & 0.2367 & -0.0434 \\
\hline & -0.0708 & -0.0434 & 0.0859 \\
\hline & 0.2635 & 0.1734 & -0.0217 \\
\hline & 0.1734 & 0.2472 & -0.0225 \\
\hline & -0.0217 & -0.0225 & 0.1555 \\
\hline & {$[0.3613$} & 0.0840 & 0.0226 \\
\hline & 0.0840 & 0.2161 & 0.0017 \\
\hline & 0.0226 & 0.0017 & 0.0890 \\
\hline & {$[0.4694$} & 0.0496 & -0.0057 \\
\hline & 0.0496 & 0.1975 & -0.0289 \\
\hline & -0.0057 & -0.0289 & 0.0412 \\
\hline
\end{tabular}

b)

Figure 5-12 - Half MBB-beam solution using PAMP TO with 5 cells and TrMIC, a) macrostructure, b) microstructure, and homogenized stiffness tensor.

\section{Tests Comparison}

Table 5-1 shows a comparison between different solutions for the MBB-beam problem. As observed, the compliance decreases when the number of materials increases. From one to three cells improves $6.22 \%$ and from three to five cells, the 
improvement is $6.31 \%$. Also, it should be noted that when the TrMIC scheme is applied there is a gain in compliance of $3.08 \%$.

Table 5-1 - Comparison of half MBB-beam using PAMP.

\begin{tabular}{|c|c|c|c|c|c|}
\hline Test & volfrac & volfrac $_{M I}$ & Number of cells & compliance & Compliance (\%) \\
\hline 1 & 0.42 & 0.6 & 1 without TrMIC & 2299 & 112.53 \\
\hline 2 & 0.42 & 0.6 & 3 without TrMIC & 2172 & 106.31 \\
\hline 3 & 0.42 & 0.6 & 5 without TrMIC & 2043 & 100 \\
\hline 4 & 0.42 & 0.6 & 5 with TrMIC & 2106 & 103.08 \\
\hline
\end{tabular}

In order to assess our method, the results were compared with those reported by Wang et al. in [55]. Figure 5-13 shows the "fully connected" solution for the MBBbeam problem, with the macro-structural topology (middle) the microstructural topology (top and bottom left), and their stiffness matrix (top and bottom right). Was reported a gain in compliance range from 4.84 to $4.85 \%$ for the different configurations tested.

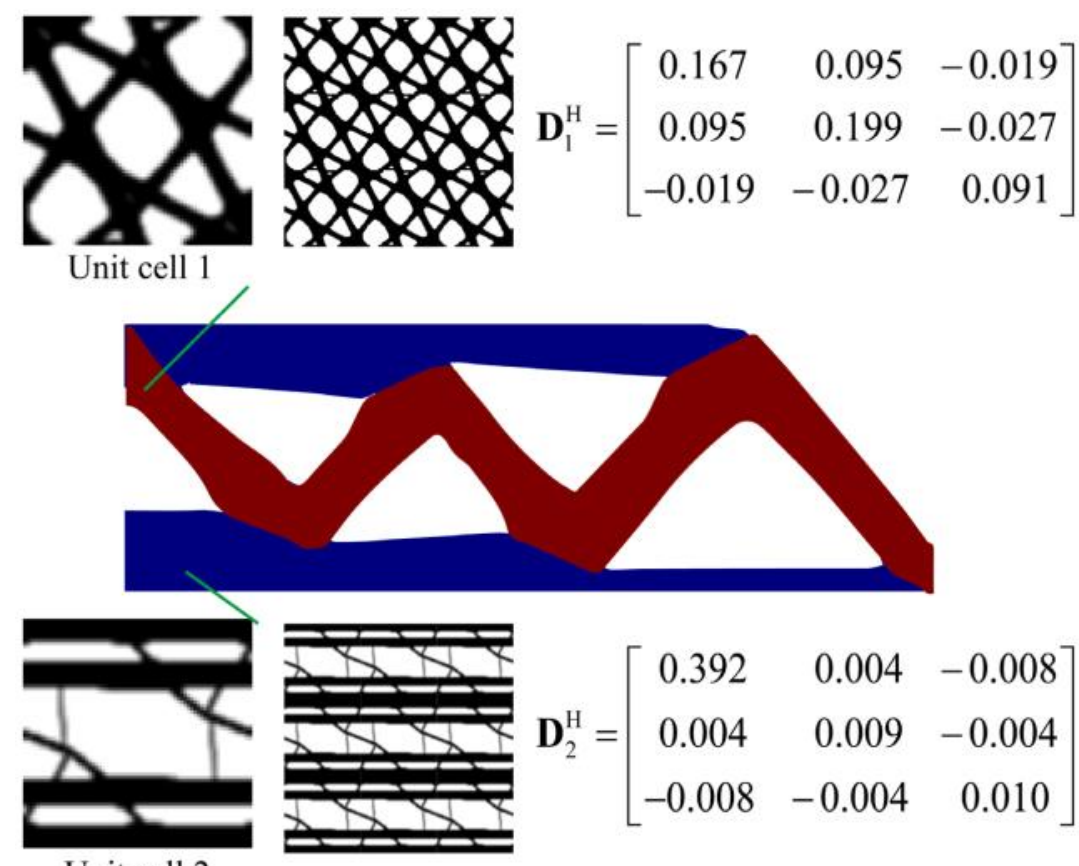

Unit cell 2

Figure 5-13 - MBB-beam solution from [55]

In both methods, is evident the loss of stiffness (gain in compliance). However, the loss in the TrMIC scheme is lower than the solution reported by Wang et al. in [55]. It should be noted that the proposed scheme does not need a parameter tuning 
process. The weighting factor $\eta$ in (50) is calculated automatically and the position of the connectivity point does not need to be predefined.

\section{2 .2}

\section{Experiments for the L-shape Problem}

\section{Test 1: Single Region Solution}

Figure 5-14 shows an L-shape problem but at this time, the force is applied at the top right corner.

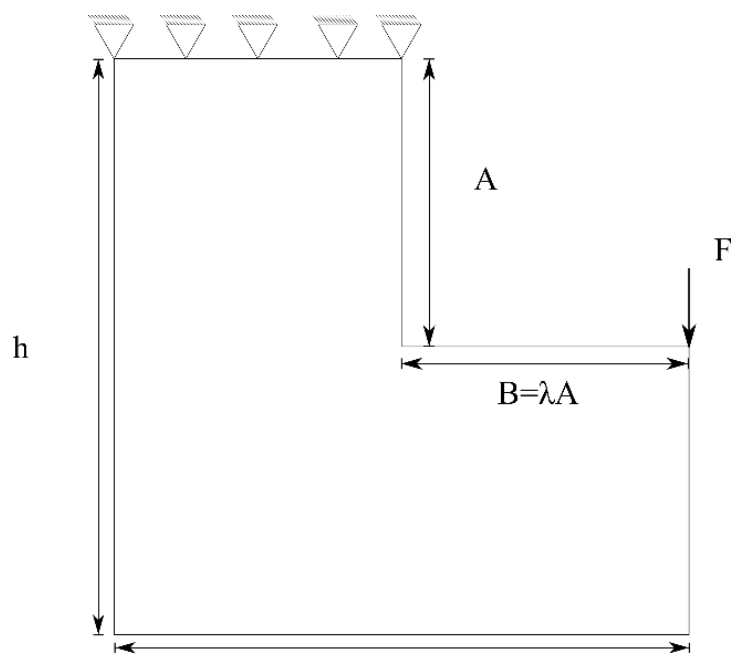

$\mathrm{L}$

Figure 5-14 - L-shape problem 2.

Figure 5-15 shows the solution of the PAMP single region, the macro and microstructure, and the homogenized stiffness tensor for the L-shape shown in Figure 5-14. With $\lambda=1$ a $h=100, L=100$ and a $A=60$ (in element`s number), a cell with $100 \times 100$ elements, volume fraction at macroscale of $\operatorname{volfrac}_{M A}=$ 0.16 , volume fraction at microscale volfrac $_{M I}=0.4$, Young's modulus $E=1$ and force $F=1$. Figure 5-15 shows the macroscale topology a), the microscale structure of which each element of the macro is composed, and the homogenized stiffness tensor b). Using the homogenized stiffness tensor the TO achieved an objective function value equals to 12967.05 


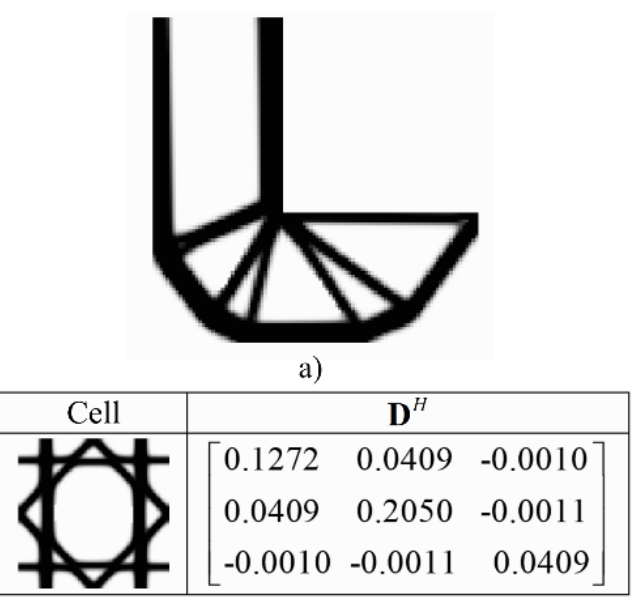

b)

Figure 5-15 - L-shape solution using PAMP. a) macrostructure, b) microstructure, c) stiffness matrix.

\section{Test 2: Multi-Region Solution for 3 Regions}

Figure 5-16 shows the solution of the PAMP multi-region with 3 different cells, there are the final macro and microscale topology and their homogenized stiffness tensor. Region one in red is composed of $60 \times 40$, region two in green by $40 \times 40$, and region three in blue by $40 \times 60$ elements. The macroscale topology a), the microscale structure b) of which each element of the macro is composed, and the homogenized stiffness tensor c). As pointed out by the orange circle (Figure 5-16 b) there are different regions where did not exists a connection between cells or the connection is poor. Using the homogenized stiffness tensor the TO achieved an objective function value equals to 9470 . 


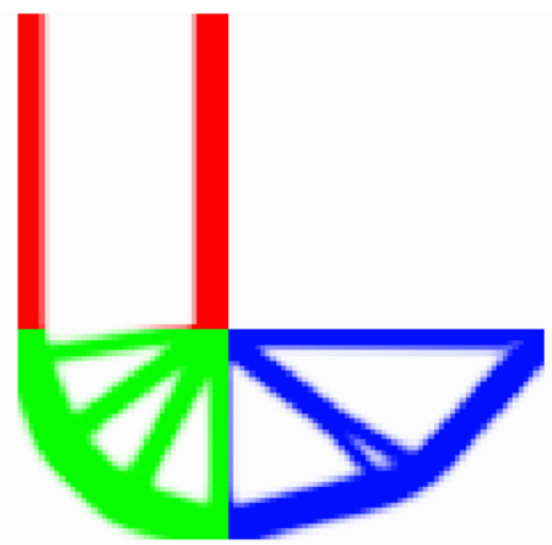

a)

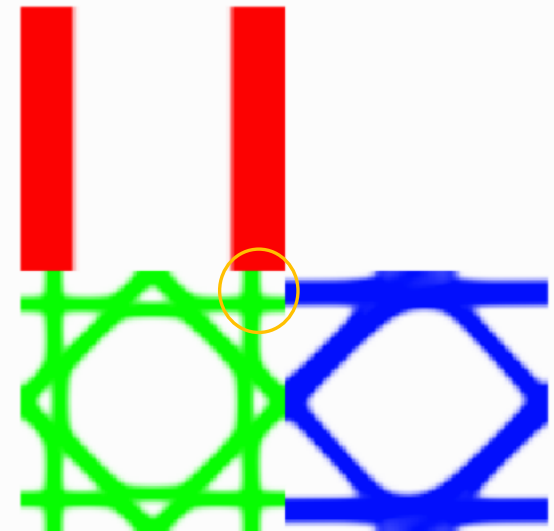

b)

\begin{tabular}{|c|ccc|}
\hline Cell & \multicolumn{3}{|c|}{$\mathbf{D}^{H}$} \\
\hline & {$\left[\begin{array}{lll}0.0000 & 0.0000 & 0.0000 \\
0.0000 & 0.3847 & 0.0000 \\
0.0000 & 0.0000 & 0.0000\end{array}\right]$} \\
\hline & {$\left[\begin{array}{lll}0.1528 & 0.0484 & -0.0011 \\
0.0484 & 0.1690 & -0.0012 \\
-0.0011 & -0.0012 & 0.0487\end{array}\right]$} \\
\hline & {$\left[\begin{array}{lll}0.2340 & 0.0570 & 0.0132 \\
0.0570 & 0.0739 & 0.0110 \\
0.0132 & 0.0110 & 0.0584\end{array}\right]$} \\
\hline
\end{tabular}

c)

Figure 5-16 - L-shape solution using PAMP with 3 cells, a) macrostructure, b) microstructure, and c) homogenized stiffness tensor. The orange circle denotes the regions where did not exists a connection between cells or the connection is poor.

\section{Test 3: Multi-Region Solution for 5 Regions}

Figure 5-17 shows the PAMP multi-region with 5 different cells, there are the final macro a) and microscale b) topology and their homogenized stiffness tensor c). Region one in red is composed for by $60 \times 40$, region two in yellow by $1 \times 40$, region three in green by $39 \times 39$, region fourth in blue by $39 \times 1$, and region five in purple is composed of $40 \times 60$ elements. As pointed out by the red circles (Figure 5-17 b) there are different regions where did not exists a connection between cells or the connection is poor. Using the homogenized stiffness tensor the TO achieved an objective function value equals to 9301. Comparing with the solution for multi-region with 3 cells shown in (Figure 5-16), the PAMP multi- 
region with 5 cells presents a $1.78 \%$ better stiffness, and a $28.9 \%$ when compared to the PAMP single cell.

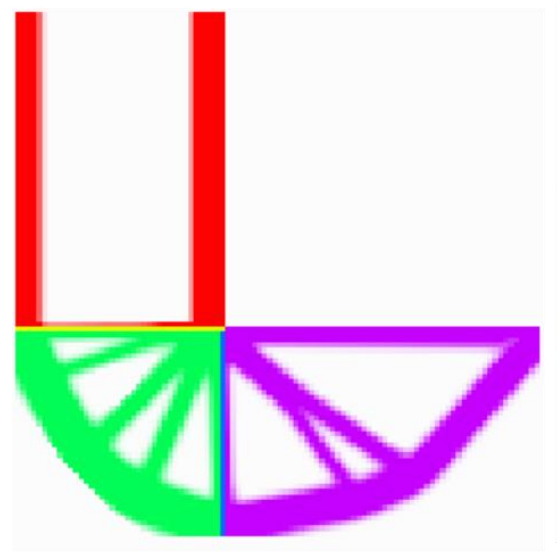

a)

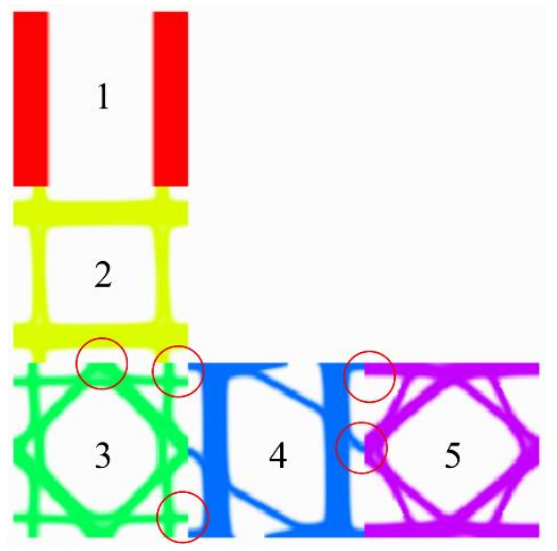

b)

\begin{tabular}{|c|c|c|c|}
\hline \multirow[t]{16}{*}{ Cell } & \multicolumn{3}{|c|}{$\mathbf{D}^{H}$} \\
\hline & 0.0000 & 0.0000 & 0.00007 \\
\hline & 0.0000 & 0.3847 & 0.0000 \\
\hline & 0.0000 & 0.0000 & 0.0000 \\
\hline & 0.2615 & 0.0165 & 0.0004 \\
\hline & 0.0165 & 0.1468 & -0.0028 \\
\hline & 0.0004 & -0.0028 & 0.0033 \\
\hline & 0.1439 & 0.0543 & -0.0092 \\
\hline & 0.0543 & 0.1651 & -0.0094 \\
\hline & -0.0092 & -0.0094 & 0.0547 \\
\hline & 0.1050 & 0.0217 & -0.0166 \\
\hline & 0.0217 & 0.2741 & -0.0103 \\
\hline & -0.0166 & -0.0103 & 0.0153 \\
\hline & 0.2188 & 0.0565 & 0.0105 \\
\hline & 0.0565 & 0.0929 & 0.0082 \\
\hline & 0.0105 & 0.0082 & 0.0568 \\
\hline
\end{tabular}

c)

Figure 5-17 - L-shape solution using PAMP with 5 cells. a) macrostructure, b) microstructure and c) homogenized stiffness tensor. The red circles denotes the regions where did not exists a connection between cells or the connection is poor.

\section{Test 4: Multi-Region for 5 Cells using TrMIC}

Figure 5-18 shows the result of the PAMP multi-region for 5 different cells, but at this time with TrMIC. There are the final macro a) and microscale topology b) 
and their homogenized stiffness tensor c), for the L-shape shown in Figure 5-17. The proposed approach was used to guarantee the connection and was imposed on the cell's regions two and four (transition cells). The principal cells $(1,4,5)$ it does not have substantial changes, but in contrast with the results, shown in Figure 5-17, not exist any disconnected point. Using the homogenized stiffness tensor the objective function value was 9358.

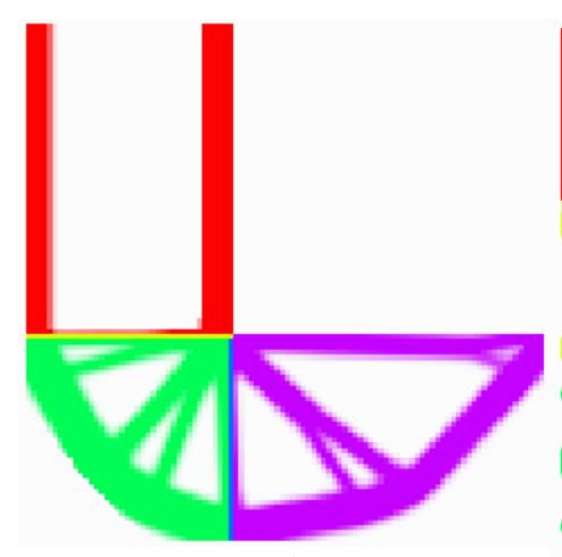

a)

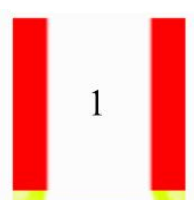

2

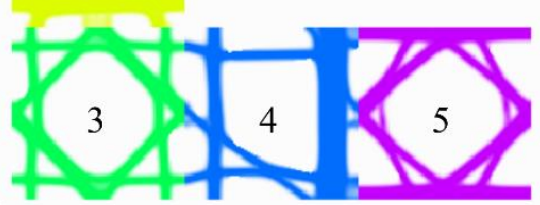

b)

\begin{tabular}{|c|ccc|}
\hline Cell & {$\left[\begin{array}{lll}0.0000 & 0.0000 & 0.0000 \\
0.0000 & 0.3847 & 0.0000 \\
0.0000 & 0.0000 & 0.0000\end{array}\right]$} \\
\hline & {$\left[\begin{array}{lll}0.2450 & 0.0195 & 0.0009 \\
0.0195 & 0.1391 & -0.0012 \\
0.0009 & -0.0012 & 0.0042\end{array}\right]$} \\
\hline & {$\left[\begin{array}{lll}0.1447 & 0.0539 & -0.0101 \\
0.0539 & 0.1656 & -0.0103 \\
-0.0101 & -0.0103 & 0.0542\end{array}\right]$} \\
\hline & {$\left[\begin{array}{lll}0.1183 & 0.0235 & -0.0170 \\
0.0235 & 0.2480 & -0.0105 \\
-0.0170 & -0.0105 & 0.0123\end{array}\right]$} \\
\hline & {$\left[\begin{array}{lll}0.2180 & 0.0561 & 0.0098 \\
0.0561 & 0.0890 & 0.0081 \\
0.0098 & 0.0081 & 0.0563\end{array}\right]$} \\
\hline
\end{tabular}

c)

Figure 5-18 - L-shape solution using PAMP with 5 cells and TrMIC. a) macrostructure, b) microstructure and c) homogenized stiffness tensor. 


\section{Tests Comparison}

Table 5-2 shows a comparison between the different solutions for the L-shape problem, As observed, the compliance decreases when the number of materials increases. From one to three cells improves $37.6 \%$ and from three to five cells, the improvement is $1.81 \%$. Also, it should be noted that when the TrMIC scheme is applied there is a gain in compliance of $0.61 \%$.

Table 5-2 - Comparison L shape PAMP.

\begin{tabular}{|c|c|c|c|c|c|}
\hline Test & volfrac & volfrac $_{M I}$ & Number of cells & Compliance & Compliance (\%) \\
\hline 1 & 0.16 & 0.4 & 1 without TrMIC & 12967 & 139.41 \\
\hline 2 & 0.16 & 0.4 & 3 without TrMIC & 9470 & 101.81 \\
\hline 3 & 0.16 & 0.4 & 5 without TrMIC & 9301 & 100.00 \\
\hline 4 & 0.16 & 0.4 & 5 with TrMIC & 9358 & 100.61 \\
\hline
\end{tabular}

To compare our the result was used those reported by Du et al. [58], they tested different values of penalization for the CI and other parameters. Figure 5-19 shows the "fully connected" solution for the L-shape problem. On the left side is shown the macro-structural topology and on the right side is shown the three cells. Was reported a gain in compliance range from 0.59 to $2.97 \%$ for the different configurations tested.

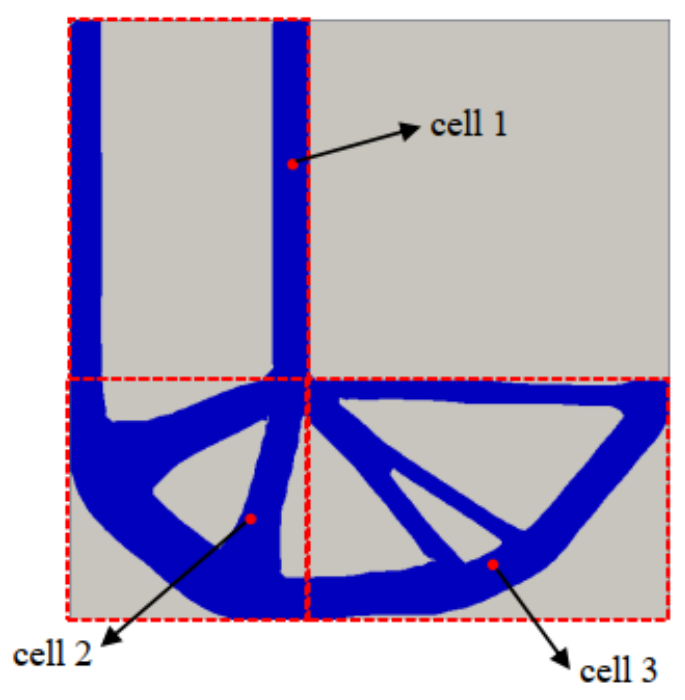

a)

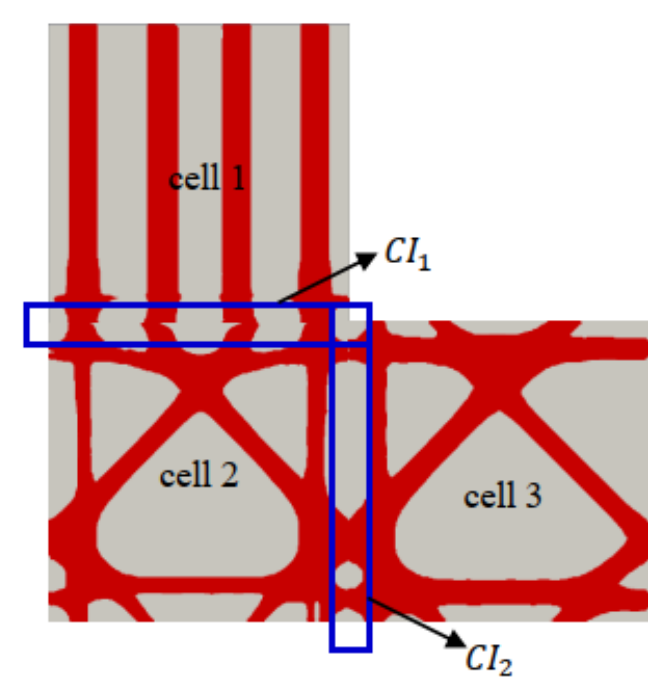

b)

Figure 5-19 - L-shape with CI solution a) macrostructure, b) from [58]. 
In both methods, is evident the loss in stiffness (gain in compliance) However, the loss in the TrMIC scheme is only $0.61 \%$. It should be noted that the TrMIC scheme does not need a parameter tuning process. The weighting factor $\eta$ in (50) is calculated automatically.

\section{3 3D Printed Samples}

The topology optimization solution present intermediate density values and therefore cannot be used directly to perform 3D printing. Hence, a post-processing treatment is require, it needs an interpretation of the model. To do this, the boundaries of final structures were obtained using the contour function from MATLAB [180] and new meshes were obtained using the open-source code mesh2d [183] for the solid (as seen in Appendix, Figure A-1 c), the graded lattice (as seen in Appendix, Figure A-2), and the PAMP with TrMIC (as seen in Figure $5-22)$.

For the 3D printed samples was used a FORMIGA P110 from the EOS company [184], and the PA2200 material. With this printer it was possible to obtain a resolution of $0.3 \mathrm{~mm}$. Figure 5-20 shows the MBB-beam solution from classic topology optimization presented in Figure A-1 (c), this object has dimensions of $18 \times 3 \times 3 \mathrm{~cm}$. The final weight of the printed part was $65.98 \mathrm{~g}$ and the density $0.93 \mathrm{~g} / \mathrm{cm}^{3}$, so the volume printed was $70.94 \mathrm{~cm}^{3}$ being the $43.79 \%$ of the bar's total volume, there is a difference of $1.79 \%$ of the original volume fraction of $42 \%$.

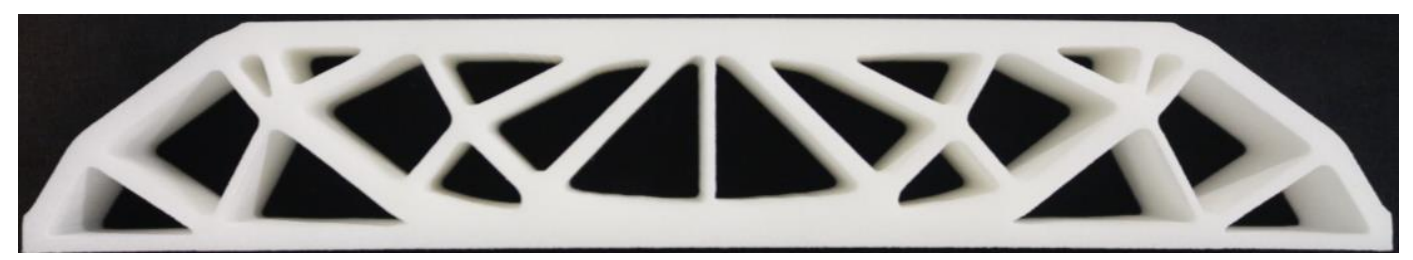

Figure 5-20 - MBB-beam solution using Classic Topology optimization.

Figure 5-21 shows the MBB-beam solution from Variable-density presented in Figure A-2. This object has a dimension of $18 \times 3 \times 3 \mathrm{~cm}$. To minimize the error in the printing process and tacking into consideration the minimal resolution of the printer, the volume fraction was set to 0.3 for every cell to avoid cross member size 
below the limit. The final weight of the printed part was $68.35 \mathrm{~g}$, the density of material $0.93 \mathrm{~g} / \mathrm{cm}^{3}$. Hence, the volume printed was $73.49 \mathrm{~cm}^{3}$ that corresponds to $45.36 \%$ which has a difference of $5.24 \%$ when compared with the original volume fraction of $40,1 \%$. It should be pointed that the original volume fraction of the variable density differs from the classical TO. This difference is due to the postprocessing of a grouping elements to replace them by the cell.

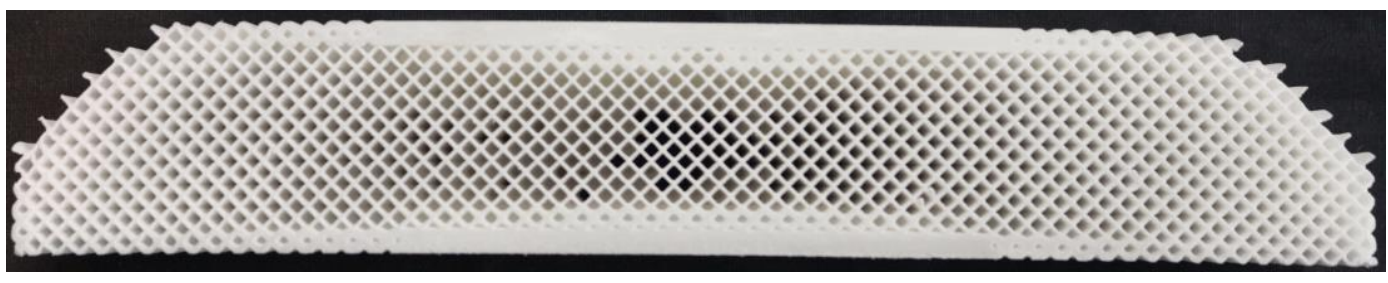

Figure 5-21 - MBB-beam solution using Variable-Density Topology Optimization.

Figure 5-22 a) shows the final arrangement for the MBB-beam solution (Figure 5-12). The cells are composed of groups of $9 \times 9$ elements with a mean volume value equal to 0.5 . Figure $5-22$ b) shows the 3D printed object with a dimension of $18 \times 3 \times 3 \mathrm{~cm}$. The final weight of the printed part was $95.06 \mathrm{~g}$, so the volume printed was $102.21 \mathrm{~cm}^{3}$ that equals to $63.09 \%$ of the total volume, and there is a difference of $19.83 \%$ of the original volume fraction of $43.26 \%$. The original volume fraction here also differs from the classic OT for the same reason of the variable-density

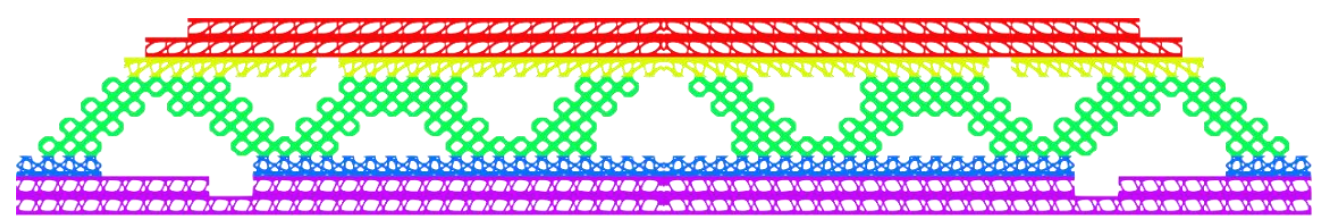

a)

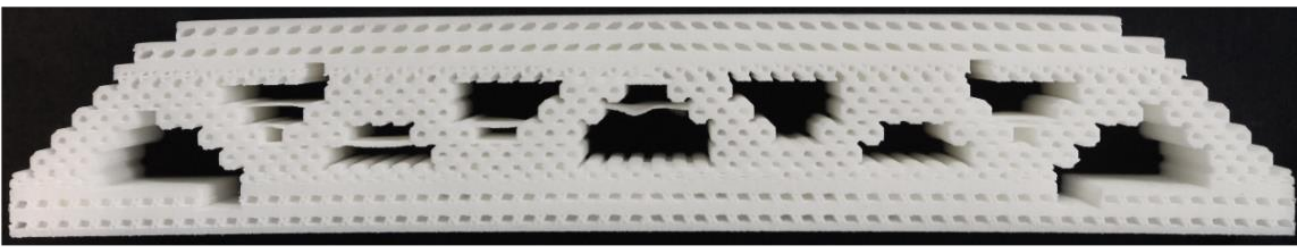

b)

Figure 5-22 - MBB-beam solution using a) PAMP multi-region TO and the 3D printed solid.

The differences in volume between the printed and the virtual design come from the slicing software and mainly for the 3D printer method in itself (the material 
clogged inside the small holes of the piece, which cannot be extracted). For this reason, was discarded. Figure 5-23 shows the physical disposition for the Threepoint flexural test, a) is the solution given by the Classical Topology optimization and $b$ ) is the Variable-density.

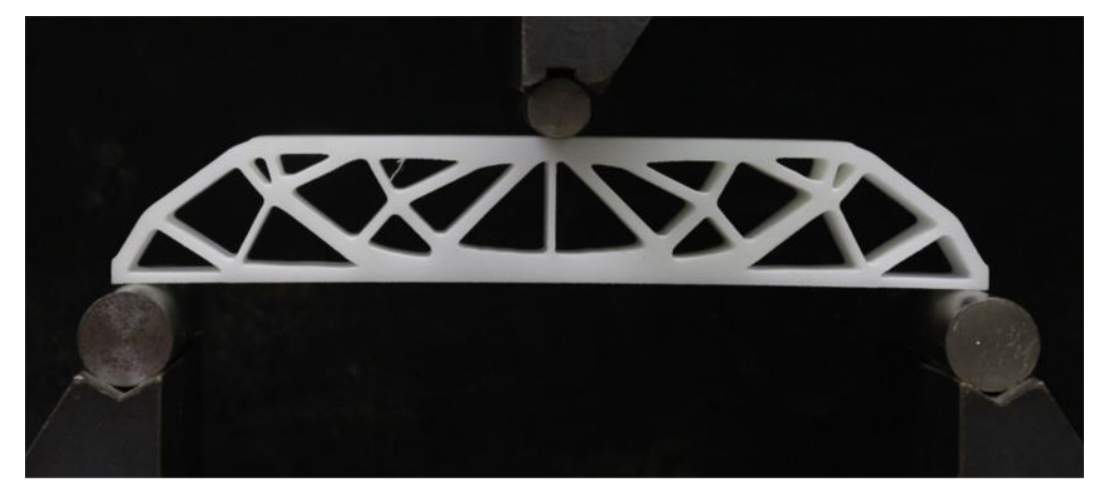

a)

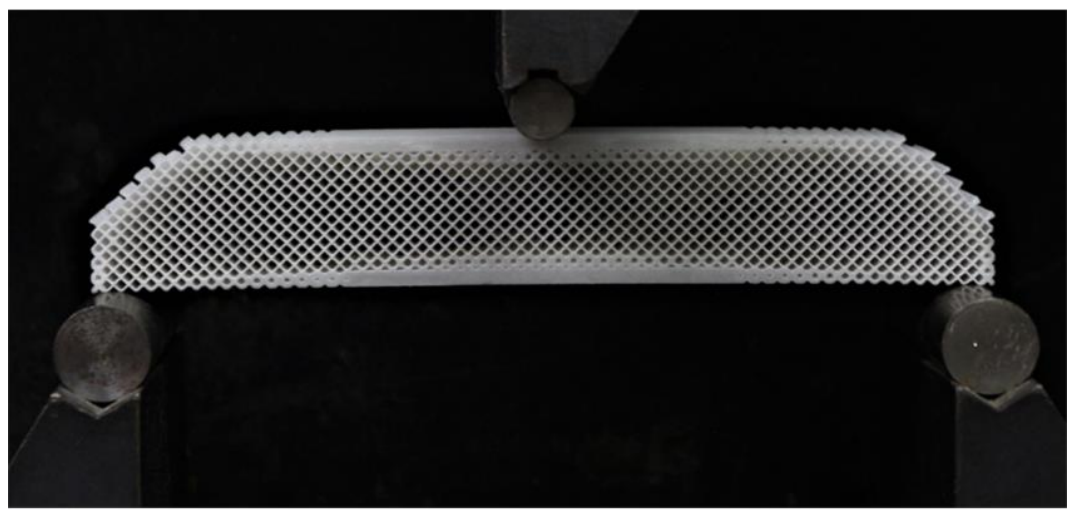

b)

Figure 5-23 - MBB-beam disposition for the three-point flexural test. a) Classic TO, b) Variable-density.

Figure 5-24 shows the data acquired from the physical tests for the samples shown in Figure 5-23. The tests were conducted in an INSTRON 1125 tensile compression tester machine at room temperature. The test velocity was set constant at $0.0331 \mathrm{~mm} / \mathrm{s}$. The reaction forces were measured with an INSTRON load cell of $25 K N$. In Figure 5-24 can be seen the classic TO, in the traced blue line. The TO reach 3141 Newton and an extension of $12.07 \mathrm{~mm}$ before collapsing. The variabledensity method, represented by the dotted gray line, reaches 1577 Newton and an extension of 17.44. The comparison between both method suggests, that the TO has a bigger stiffness and better energy absorption. 


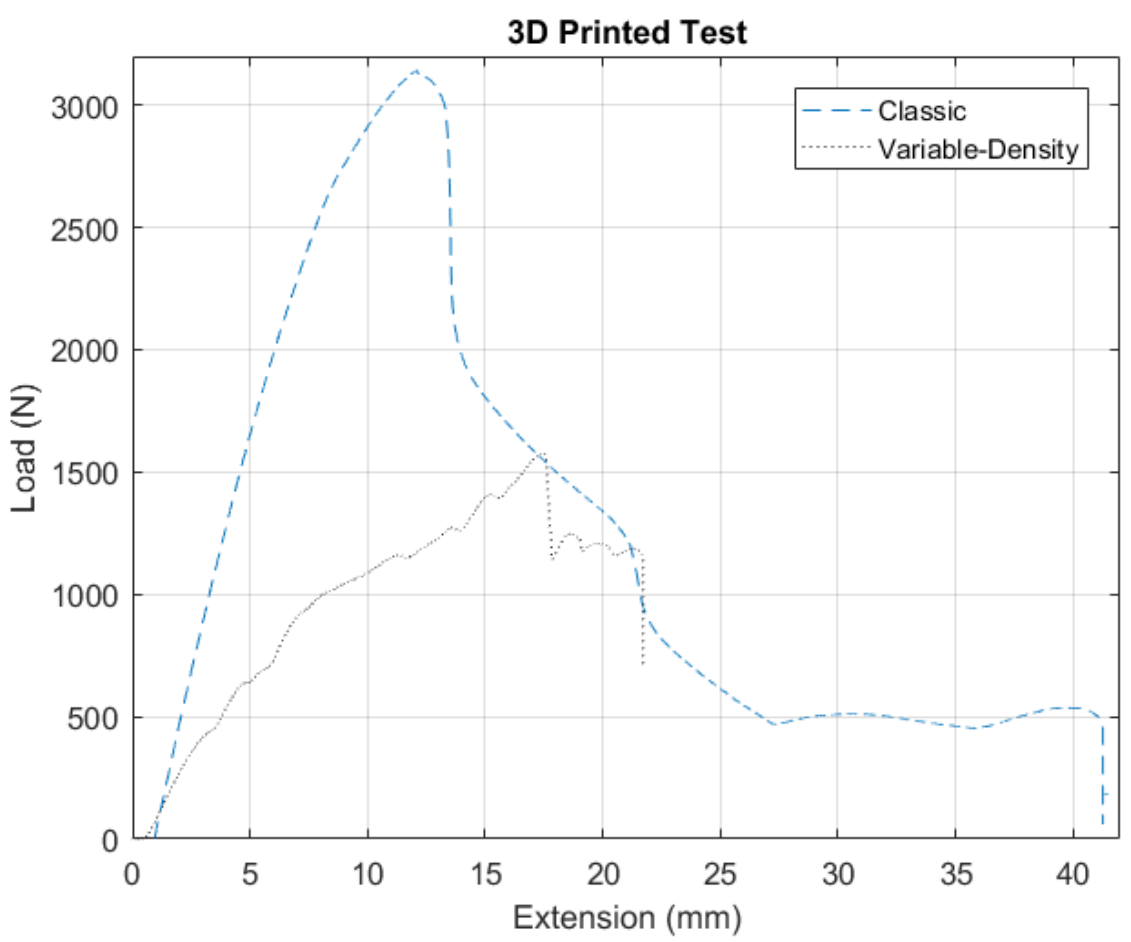

Figure 5-24 - Three points flexural test for classic and Variable-density 3D printed samples.

As expected, the classic (solid structure) has superior stiffness than the porous one $[44,48,84,85,90,185,186]$. On the other hand, the porous structures tend to have increased buckling resistance $[52,55,187]$ that allows reaching more than four times the buckling load and high energy absorption [86, 90]. Moreover, as reported in [188], support material was not required for the porous structure. Additionally, by not needing a post-processing step, it is possible to avoid damaging the piece. Figure 5-25 shows the results given by Ultimaker Cura [189] software where the support material is represented by red color.

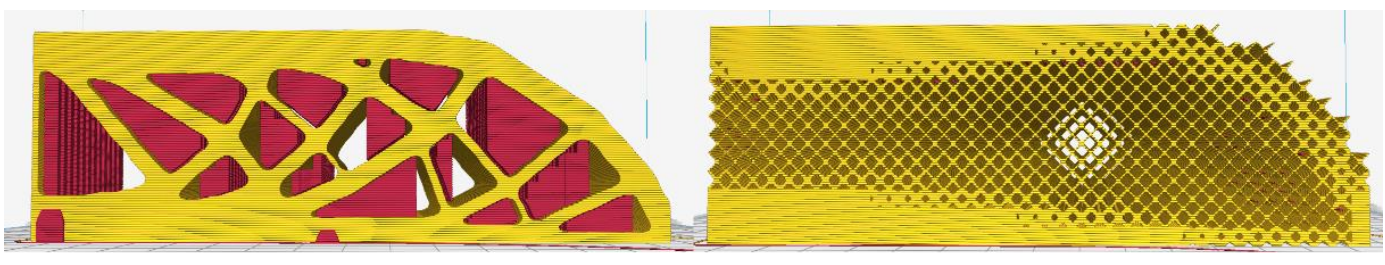

a)

Figure 5-25 - Simulation of a 3D printed solid from [188]. 


\section{4}

\section{ANSYS Simulation}

This section presents the FEM simulation of the previously 3D printed models. The analysis was performed on a machine with an Intel® Core i9-7920X, @ 2.90GHz (24 CPUs) processor and 64 GB of RAM, an NVIDIA GeForce GT 710 with 1GB of RAM, and was use ANSYS 2019R1 Academic Version.

\section{4 .1}

\section{Classic TO}

The analysis was configured as illustrated in Figure 5-26: a) an applied force of $2250 \mathrm{~N}$, increasing in steps of $50 \mathrm{~N}$ in $\mathrm{Y}$ direction. The area where the force is applied is in the middle of the upper face and has an area of $6 \times 30 \mathrm{~mm}$; b) shows the displacement lock that works as supports and both have an area of $6 \times 30 \mathrm{~mm}$.

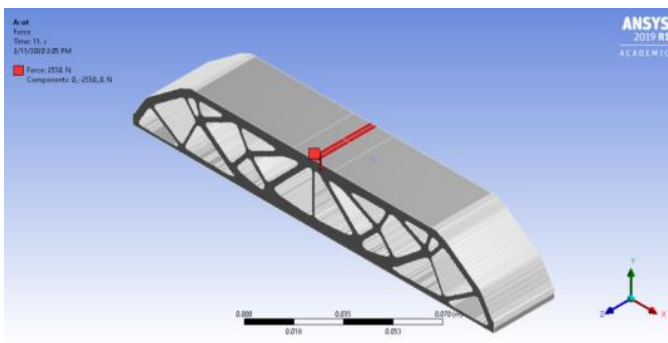

a)

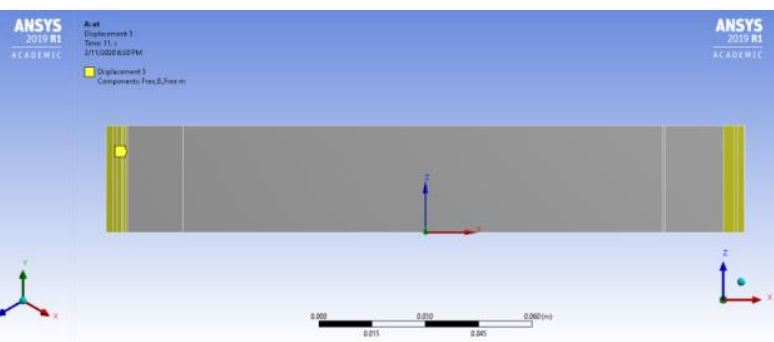

b)

Figure 5-26 - ANSYS force and supports and force for Classic TO analysis.

Figure 5-27 shows in a) the restriction of the model to prevent the displacement in the $\mathrm{Z}$-axis applied in the lateral face and $\mathrm{b}$ ) is the restriction to prevent the displacement in $\mathrm{X}$-axis.

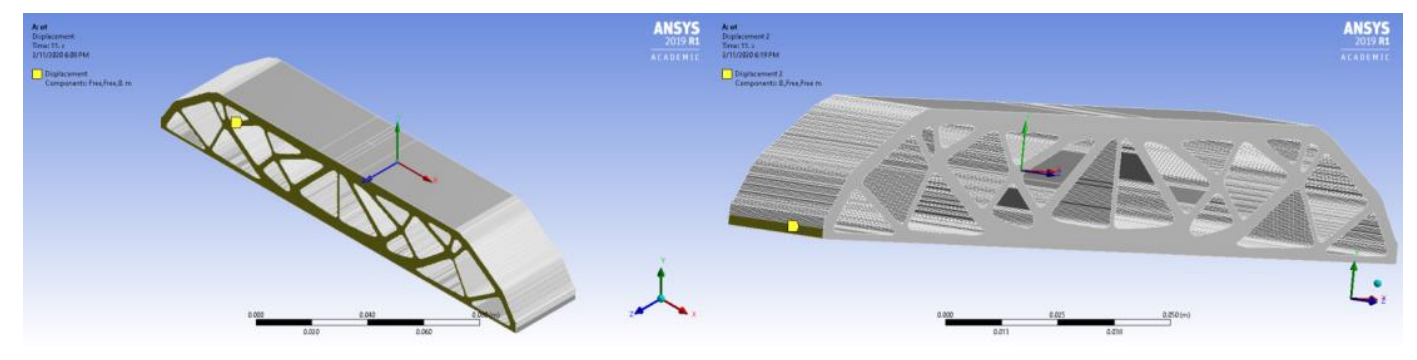

a) b)

Figure 5-27 - ANSYS boundary conditions for Classic TO analysis. 
To mesh the model was used a quadratic element, the size was set as default with a resolution of two (2) in mesh sizing. Four mesh refinements were employed, three on the faces and one on the edge, as shown in Figure 5-28. The final number of nodes was 1868316 and 917490 elements.

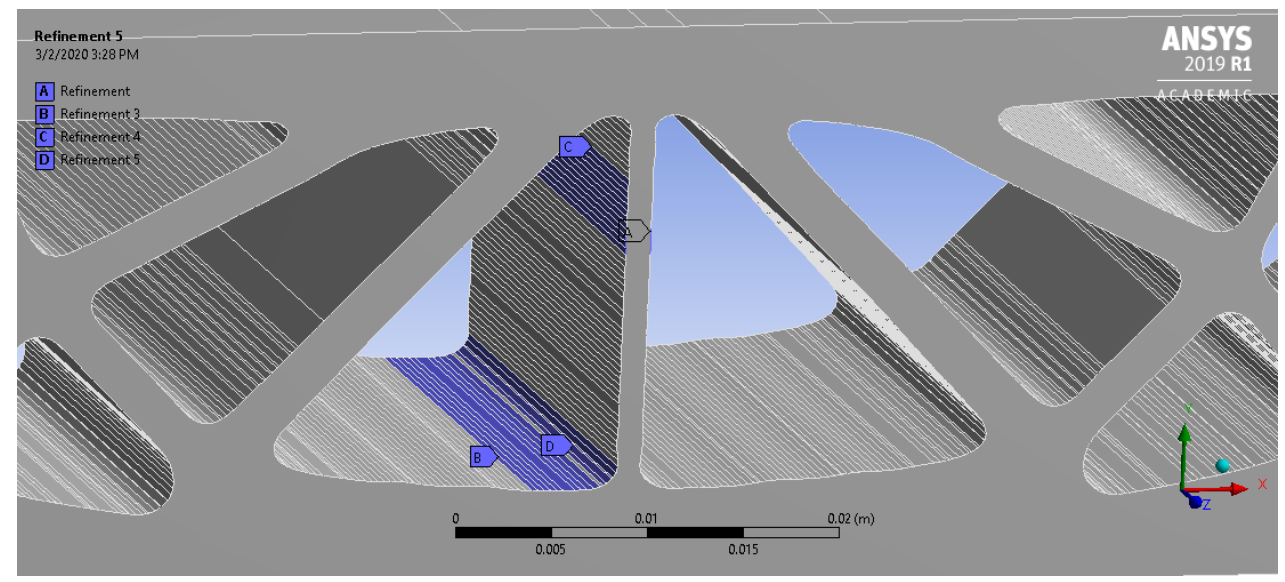

Figure 5-28 - ANSYS Mesh refinement for TO classic.

For the mechanical properties was used a bilinear isotropic material as described in Table 5-3, some of these values were taken from the datasheet provided by the EOS company[184].

Table 5-3 - Material Properties for ANSYS.

\begin{tabular}{|c|c|}
\hline Property & Value \\
\hline Density $\rho$ & $0.93 \mathrm{~g} \cdot \mathrm{cm}^{-3}$ \\
\hline Young's Modulus $E$ & $1700 \mathrm{MPa}$ \\
\hline Poisson's ratio $v$ & 0.33 \\
\hline Yield Strength & $24 \mathrm{MPa}$ \\
\hline Tangent Modulus & $48 \mathrm{MPa}$ \\
\hline
\end{tabular}

For the solver controls in the Analysis Settings, was used the "Direct" option and the "large deflections" option was activated, the restarts points were left to the program controller, and for the nonlinear controls, the "Newton-Raphson" option was activated as "full".

Figure 5-29 shows the ANSYS solution for the Von-Mises stress for the classic $\mathrm{TO}$, there is shown maximum stress of $35.966 \mathrm{MPa}$ and a minimum value of 0.020 $\mathrm{MPa}$. 


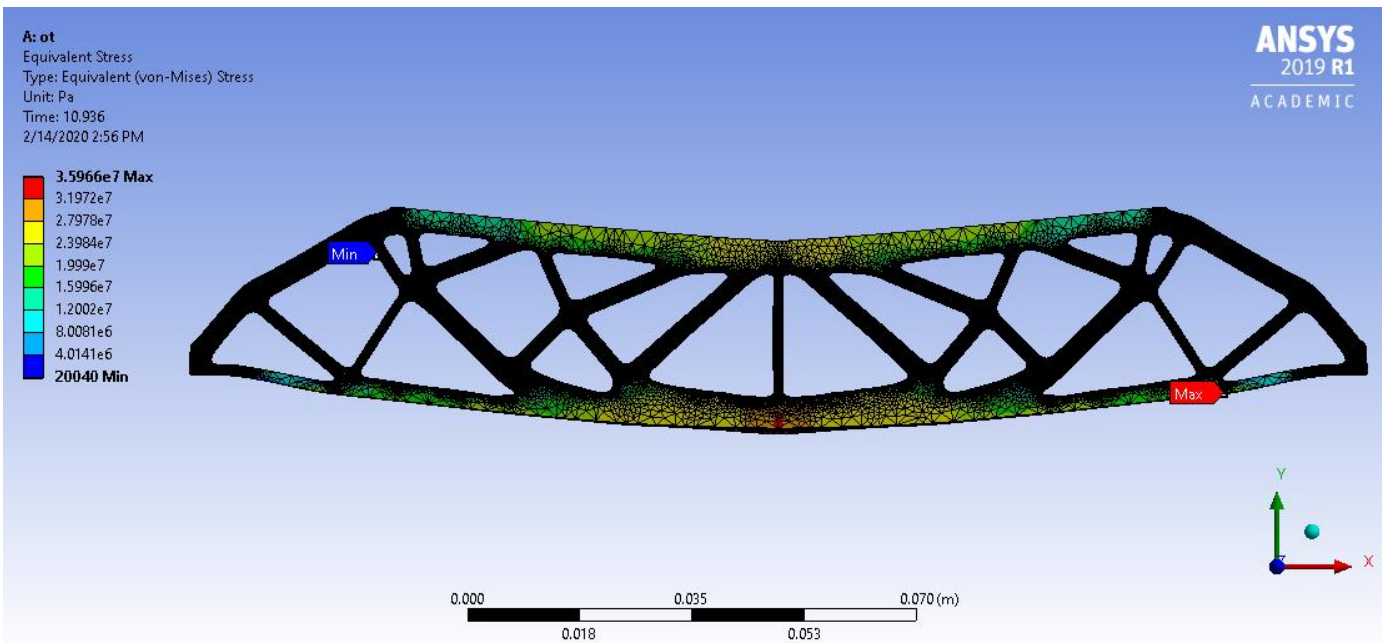

Figure 5-29 - ANSYS Von-Mises stress for Classic TO.

Figure 5-30 shows the total strain for the classic TO. With a maximum of $0.19051 \mathrm{~m} / \mathrm{m}$ (red color) and a minimum of $1.3315 \times 10^{-5} \mathrm{~m} / \mathrm{m}$ (blue color).

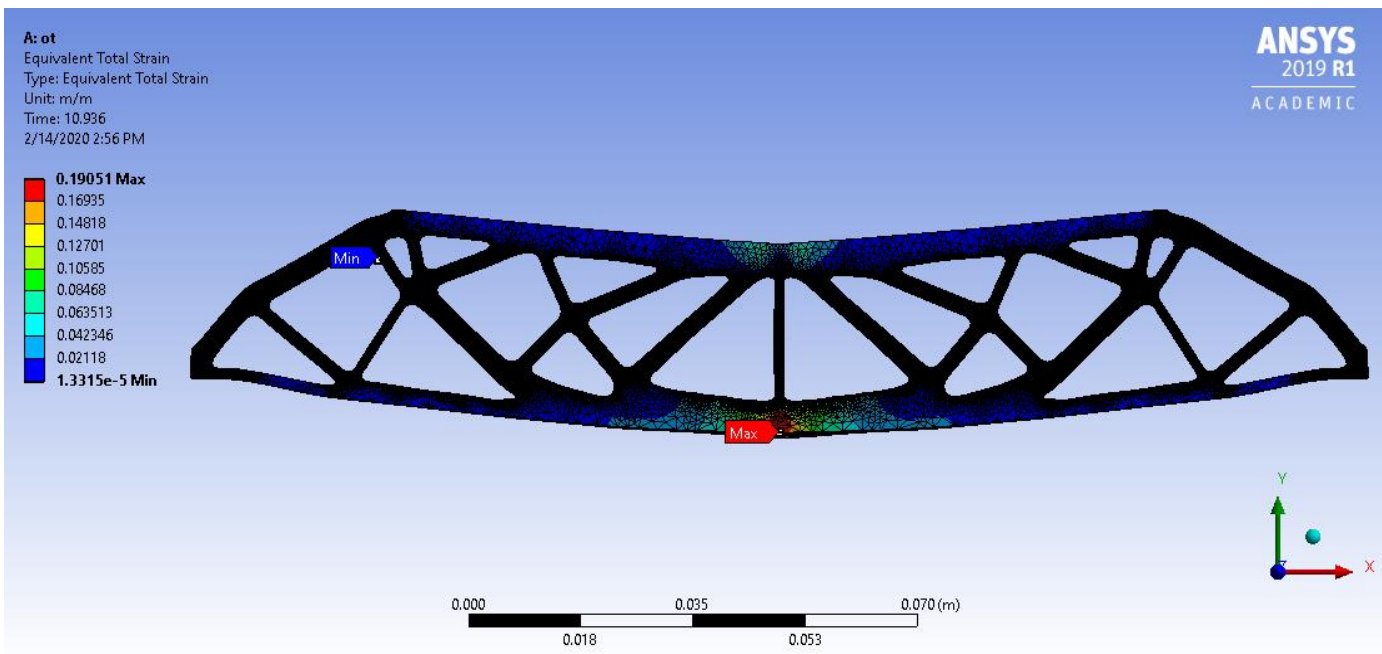

Figure 5-30 - ANSYS total strain for Classic TO.

Figure 5-31 shows the maximum deformation reached, this deformation occurs on the region where the load is applied with a value of $9.8577 \times 10^{-3} \mathrm{~m}$, and a minimum deformation value of $5.055 \times 10^{-8}$ located in the support regions. 


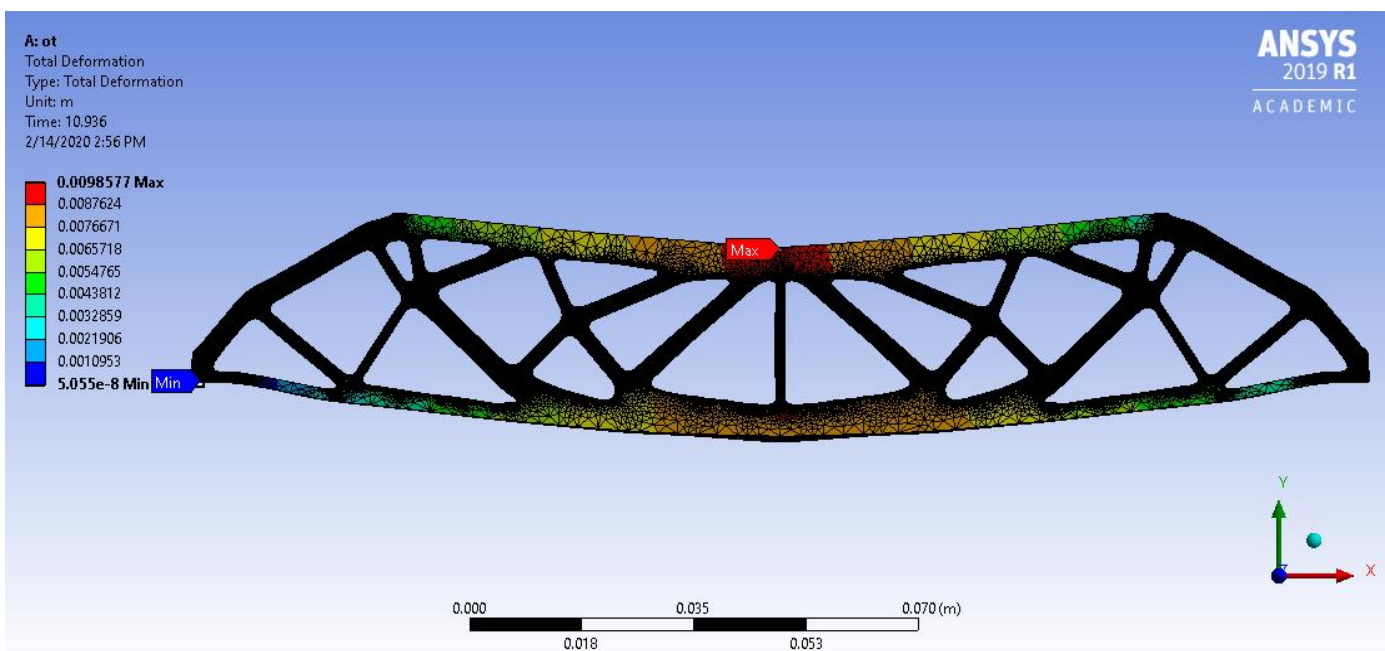

Figure 5-31 - ANSYS total deformation for Classic TO.

Figure 5-32 shows the physical test for the classic TO (the solid blue line) and the result from the ANSYS simulation (dotted red line) the simulation was done with the data provided by the printer manufacturer, the discrepancy between the 2 lines is expected because of the uncertainty of the material properties and the $3 \mathrm{~d}$ printed process itself.

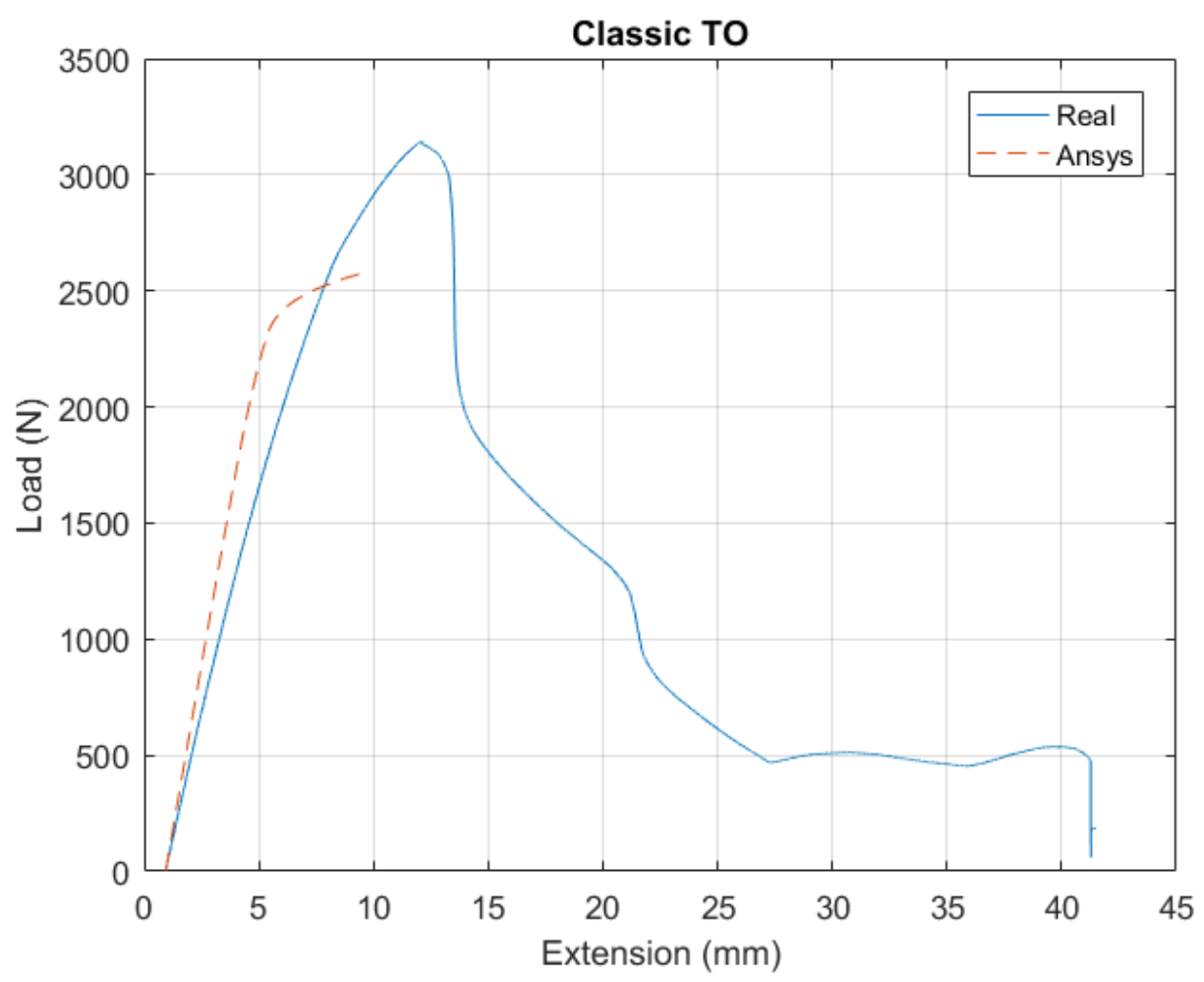

Figure 5-32 - Comparison TO. 


\section{4 .2}

\section{Variable-density}

The analysis was configured as shown in Figure 5-33. a) shows an applied force of $1200 \mathrm{~N}$ increasing in steps of 50 by 50 newton in the $\mathrm{Y}$ direction. The area where the force is applied is in the middle of the upper face and has an area of $6 \times 30 \mathrm{~mm}$. b) is the displacement restriction that works as supports in the first three faces of the two first cells.

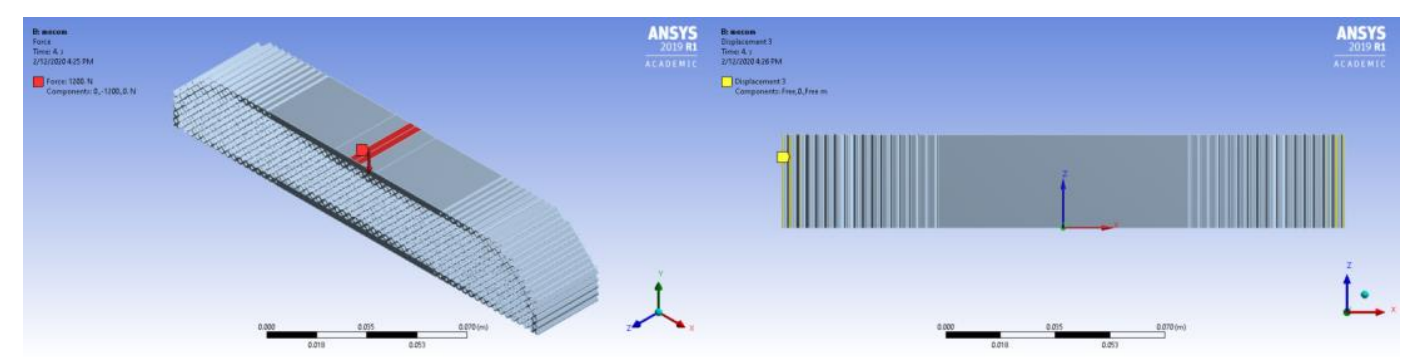

a)

b)

Figure 5-33 - ANSYS force and support for Variable-density TO.

Figure 5-34 shows a) the restriction of the model to prevent the displacement in the Z-axis applied in the lower side edge and b) the restriction to prevent the displacement in $\mathrm{X}$-axis applied in the first face on the first cell.

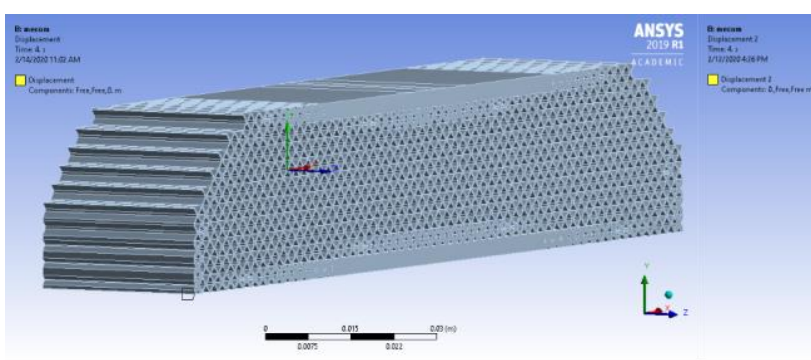

a)

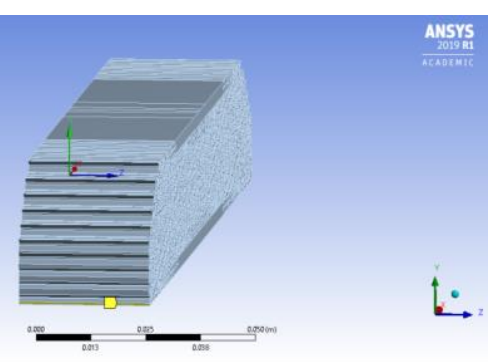

b)

Figure 5-34 - ANSYS boundary conditions for Variable-density TO.

To mesh the model was used a quadratic element, the size was set as default with a resolution of four (4) in mesh sizing and any mesh refinements were used. The final number of nodes was 2945971, and for the number of elements was 539640, the mechanical properties used were the same as in Table 5-3. For the solver controls in the Analysis Settings, was used the "Direct" option and the "large 
deflections" option was activated, the restart points were left to the program controller, and for the nonlinear controls, the "Newton-Raphson" option was setting as "full”.

Figure 5-35 shows the ANSYS solution for the von-Mises stress for the Variable-density TO. There is shown maximum stress of $69.597 \mathrm{MPa}$ and a minimum value of $0.0317 \mathrm{MPa}$, which are located near the supports, also can see a stress concentration in the same region (highlighted in the red circle).

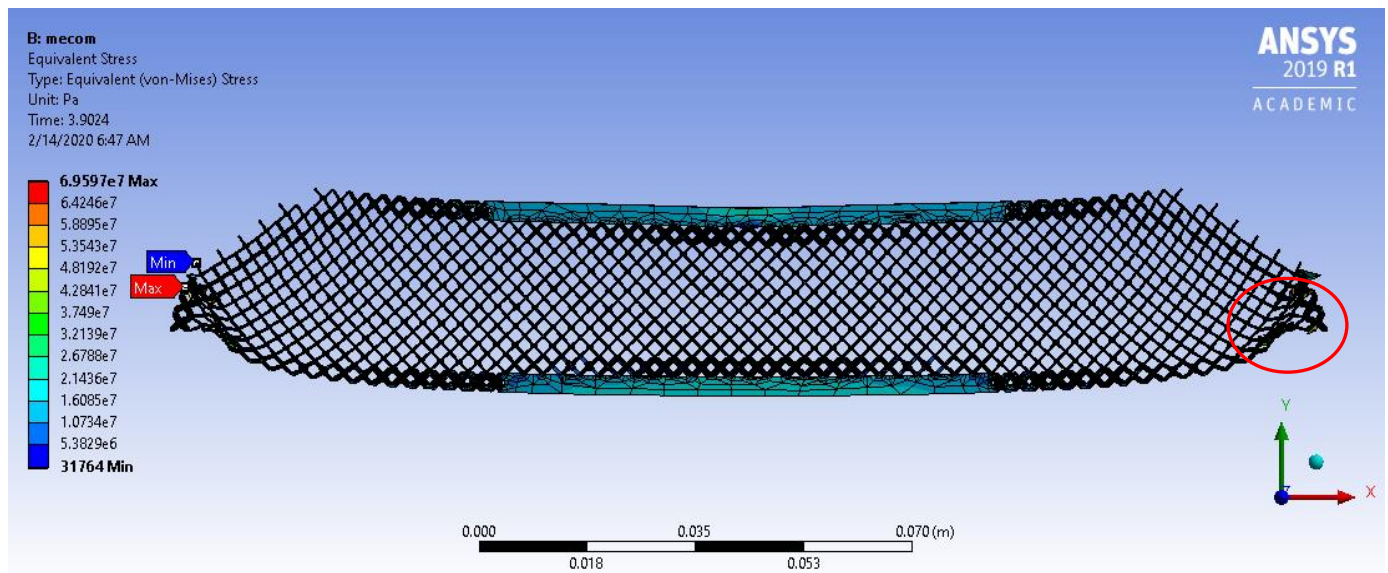

Figure 5-35 - ANSYS Von-Mises stress for Variable-density.

Figure 5-36 shows the total strain for the Variable-density TO with a maximum of $0.898 \mathrm{~m} / \mathrm{m}$ and a minimum of $1.938 \times 10^{-5} \mathrm{~m} / \mathrm{m}$, both located near the support.

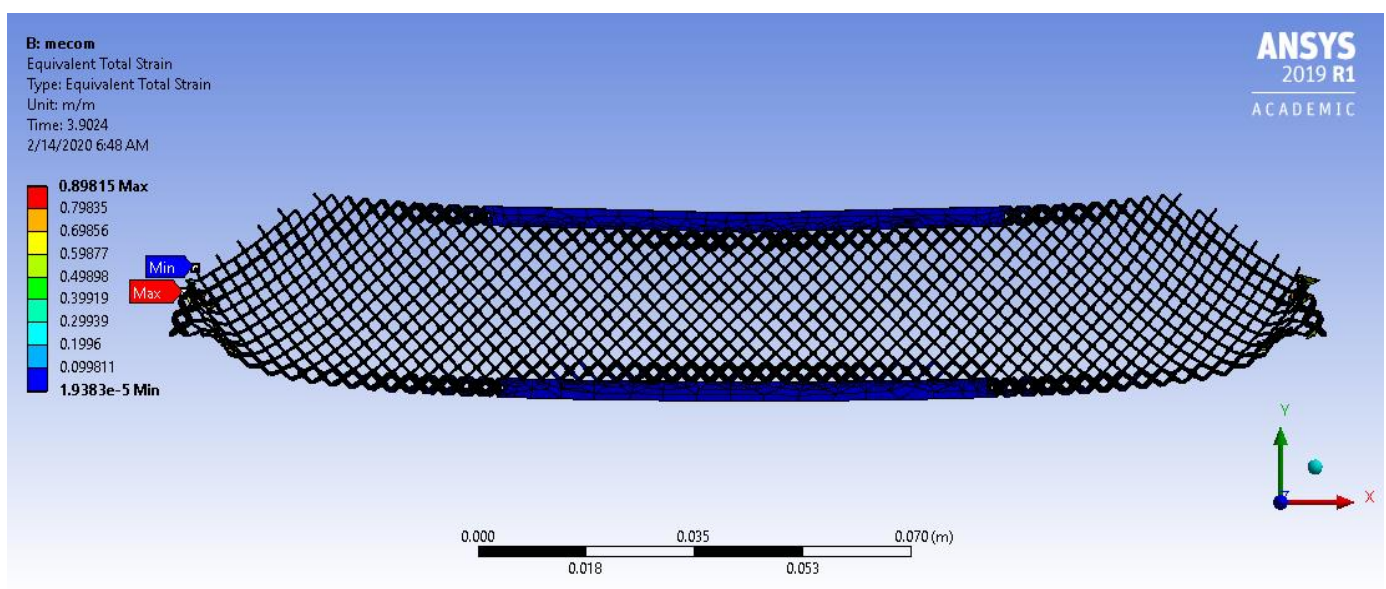

Figure 5-36 - ANSYS equivalent total strain for Variable-density.

Figure 5-37 shows the maximum deformation reached in the model, this deformation is just in the region where the load is applied with a value of $11.09 \times$ 
$10^{-3} \mathrm{~m}$, and a minimum deformation value of $2.442 \times 10^{-6}$ located in the support regions.

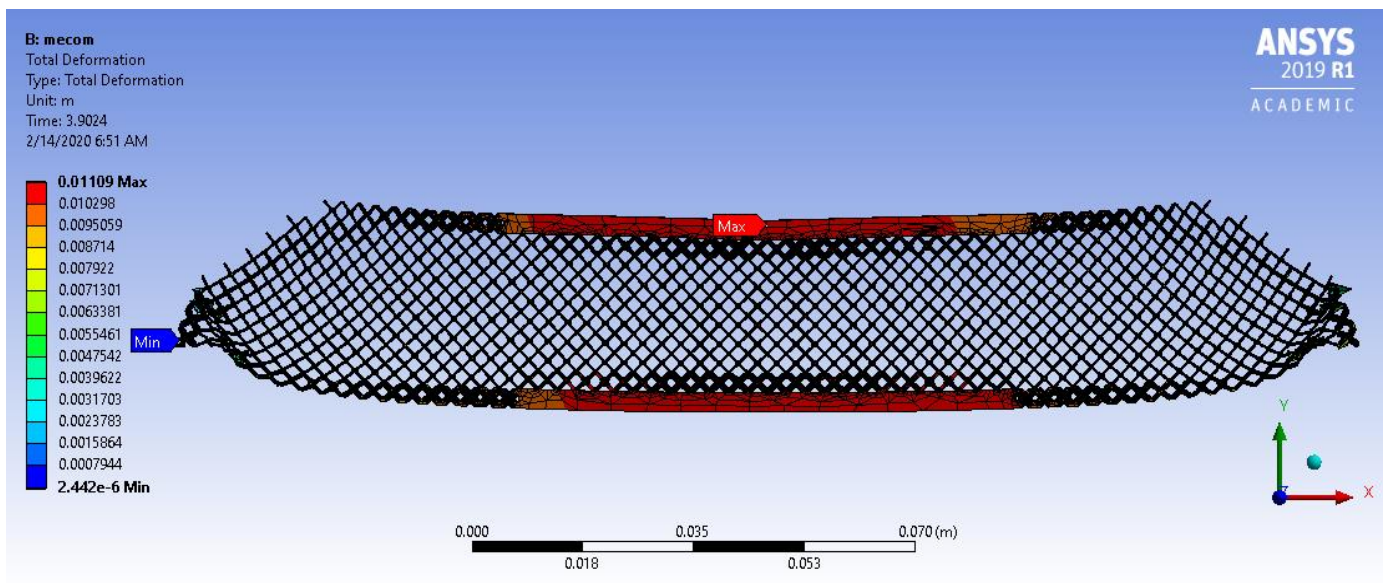

Figure 5-37 - ANSYS total deformation for Variable-density.

In order to get a reliable mesh convergence analysis was conducted by considering the number of elements and the maximum equivalent stress. Figure 5-38 summarizes the results of the analysis base on the number of elements. There can see that the number of element rise to $5 \times 10^{5}$, but converges at $3.5 \times 10^{5}$.

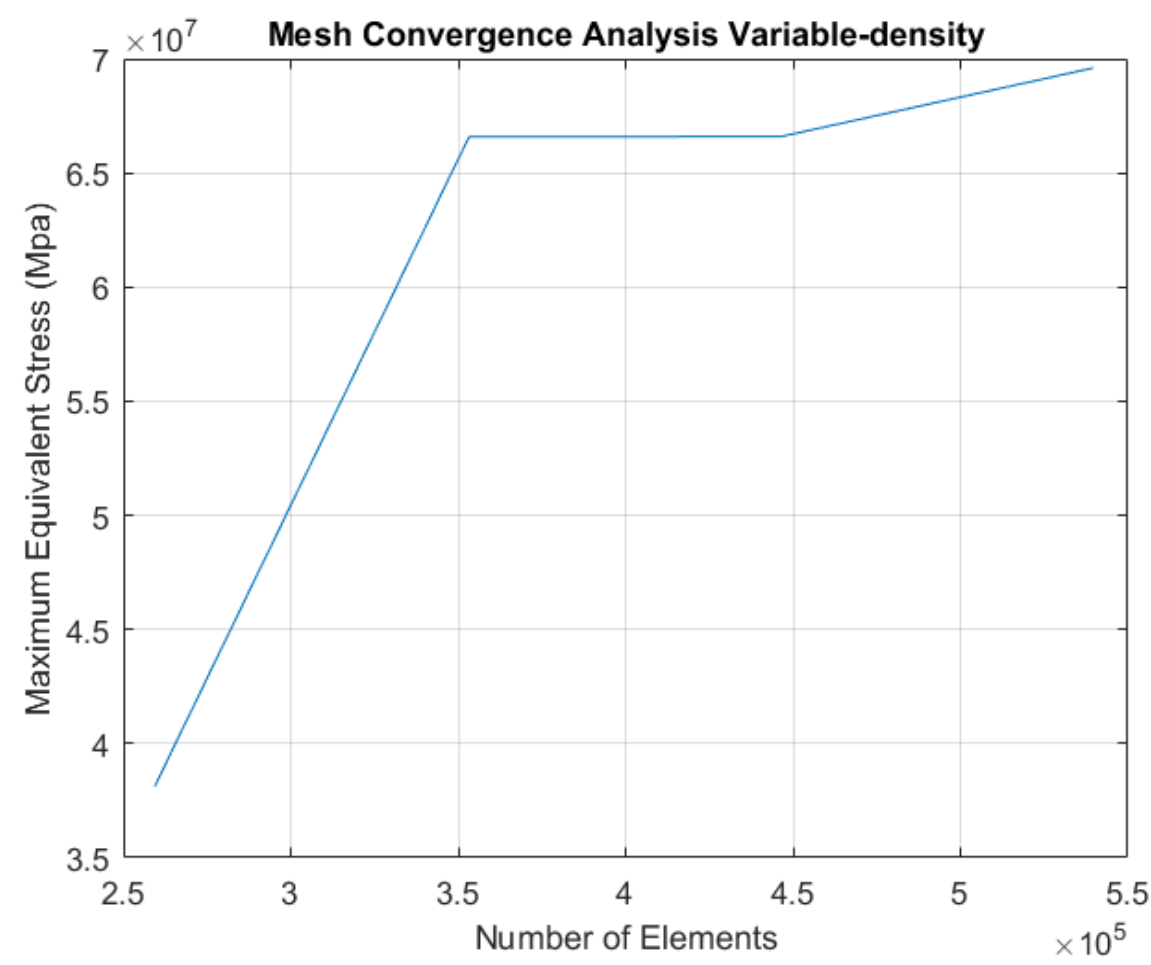

Figure 5-38 - ANSYS mesh convergence analysis for Variable-density. 
Figure 5-39 shows the physical test for the Variable-density TO (the solid blue line) and the result from the ANSYS simulation (dotted red line). The discrepancy between the 2 lines is expected because of the uncertainty of the material properties and the $3 \mathrm{~d}$ printed process itself.

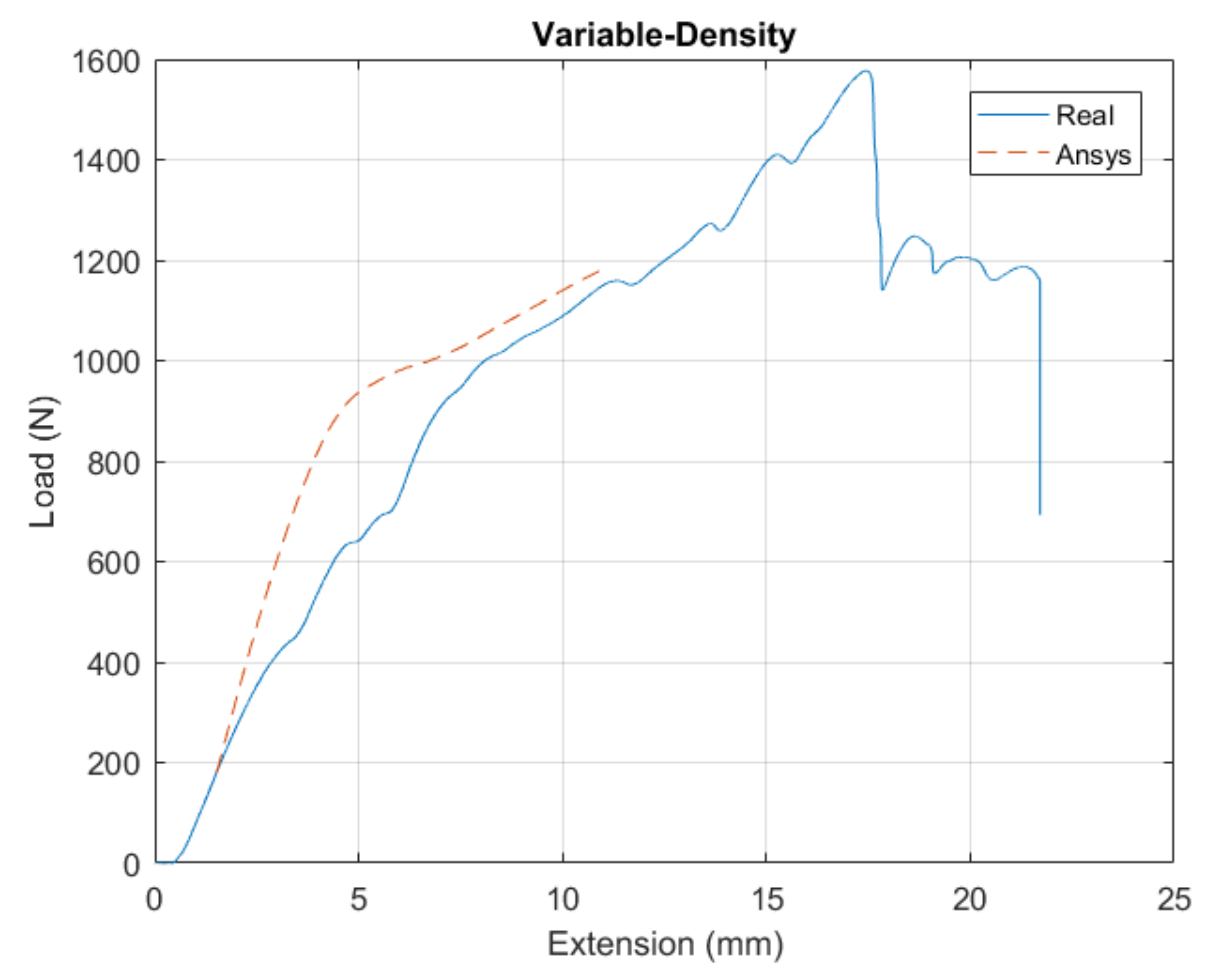

Figure 5-39 - Comparison Variable-Density.

Table 5-4 shows the maximum load and extension reached for the classic and the Variable-density method, for the real test and the ANSYS simulation. The maximum load (\%) reached is near to $50 \%$ but the maximum extension is different because the elements in the Variable-density are highly distorted, which suggests that a better mesh treatment must be performed.

Table 5-4 - Comparison of 3D printed models, physical and ANSYS tests.

\begin{tabular}{|c|cccc|cccc|}
\hline \multicolumn{7}{|c|}{ Real Test } & \multicolumn{5}{c|}{ ANSYS } \\
$\begin{array}{c}\text { Sample } \\
\text { Test }\end{array}$ & $\begin{array}{c}\text { Max. } \\
\text { Load } \\
(\mathrm{N})\end{array}$ & $\begin{array}{c}\text { Max. } \\
\text { Load } \\
(\%)\end{array}$ & $\begin{array}{c}\text { Max. } \\
\text { Extension } \\
(\mathrm{mm})\end{array}$ & $\begin{array}{c}\text { Max. } \\
\text { Extension } \\
(\%)\end{array}$ & $\begin{array}{c}\text { Max. } \\
\text { Load } \\
(\mathrm{N})\end{array}$ & $\begin{array}{c}\text { Max. } \\
\text { Load } \\
(\%)\end{array}$ & $\begin{array}{c}\text { Max. } \\
\text { Extension } \\
(\mathrm{mm})\end{array}$ & $\begin{array}{c}\text { Max. } \\
\text { Extension } \\
(\%)\end{array}$ \\
\hline $\begin{array}{c}\text { Classic } \\
\text { TO } \\
\begin{array}{c}\text { Variable- } \\
\text { density }\end{array}\end{array}$ & 3141 & 100 & 12.07 & 100 & 2582 & 100 & 9.76 & 100 \\
\hline
\end{tabular}




\section{6 \\ Conclusions and Future Works}

The principal contribution of this work is the development of an effective compatibility scheme in the context of multiscale topology optimization. The scheme is capable of generating smooth, well-connected transitions between microstructures composed of unit cells that may not have clear connectivity. It uses the kinematical connector method and the two-step pseudo-load-base method where the pseudo loads are gradually applied in two opposite parallel surfaces under a non-periodic boundary condition.

To accomplish this, numerical experiments were conducted on topology optimization in single-scale and multiscale, as well as different techniques to guarantee a smooth and continuous geometric transition between cells in multiregion porous solid. The Solid Isotropic Material with Penalization (SIMP), a single-scale method, and the Porous Anisotropic Material with Penalization (PAMP), a multiscale method, were presented.

The PAMP method was expanded to multi-region using the Discrete Material Optimization (DMO) approach. The Asymptotic homogenization and the energybased approaches were used to compute the equivalent properties of microstructures. The Kinematical Connector and two steps Pseudo Load methods were used as connection methods.

The numerical results presented is this work were obtained using a MATLAB implementation based on 88-lines code [143] and topX code [32, 172]. The proposed scheme was tested using two popular problems in the literature: The Lshape beam, and the MBB-beam. These tests suggested that the stiffness of the PAMP multi-region is strongly influenced by the number of regions and it is evident in the results shown in Table 5-1 and Table 5-2. As we increase the number of regions the structure become more efficient in terms of stiffness. The structure which has more regions are able to better represent the varying directions and magnitudes of the principal stresses. 
The proposed approach is presented as an effective method to generate smooth connections between adjacent cells. The approach presented a drop of $0.61 \%$ and $3.08 \%$ in terms of stiffness. With all, the proposed scheme has equal or lower loss compared with others methods. Nonetheless, the drop in stiffness could be solved by relaxing the restriction in the TrMIC, for example a higher volume.

To further illustrate the multiscale approaches presented in this work, the MBB beam structure was printed out via additive manufacturing and a three-point bending beam mechanical testing was performed. Three different solutions to the MBB-beam problem were considered, i) the classic topology optimization, ii) the variable-density, and iii) the PAMP multi-region with 5 different cells using the proposed scheme. The Formiga P110 3D printer from the EOS company was used, with the polymeric compound PA2200 and the SLS technology. Due to difficulties in removing the trapped powder from the PAMP build, we were unable to perform the mechanical test in this specimen.

From the experimental results, we can see that the variable-density structure has much higher compliance than the solid, isotropic case. Although it was expected an increase in the compliance, the higher value obtained here is due to the failure of lattice cell crushing observed around the support points. This behavior is also observed in the numerical analysis using ANSYS for the classic and Variabledensity solutions, as shown in Figure 5-32, Figure 5-39, and Table 5-4. Also, as seen in Figure 5-35, Figure 5-36, and Figure 5-37.

\section{1}

\section{Future Work}

As future work, the results of this thesis indicate several points for improvement:

- Since the performance of Multiscale TO with multiple regions depends on where the cellular regions are located and the amount of them. It is suggested to design a method to designate automatically the position and number of the different regions such as the approach presented in [190].

- To avoid the trapped powder inside the printed object, it is suggested to constrain the minimum size for the void region in the microscale. 
- Since the optimization process was subjected to a volume constraint without considering other parameters such as the maximum stress, the solution can suffer stress above the proportional limit. To avoid this, it is suggested to add a stress restriction in the optimization design [17]. 


\section{References}

1. Wegst UGK, Bai H, Saiz E, et al (2015) Bioinspired structural materials. Nat Mater 14:23-36. https://doi.org/10.1038/nmat4089

2. Lakes $R$ (1993) Materials with structural hierarchy. Nature 361:511515. https://doi.org/10.1038/361511a0

3. Gibson LJ, Ashby MF (1997) Cellular solids structure and properties. Cambridge University Press

4. Mazur M, Leary M, McMillan M, et al (2017) Mechanical properties of Ti6Al4V and AISi12Mg lattice structures manufactured by Selective Laser Melting (SLM). In: Laser Additive Manufacturing. Elsevier, pp 119-161

5. Hohe J, Hardenacke V, Fascio V, et al (2012) Numerical and experimental design of graded cellular sandwich cores for multifunctional aerospace applications. Mater Des 39:20-32. https://doi.org/10.1016/J.MATDES.2012.01.043

6. R Lakes (1996) Cellular solid structures with unbounded thermal expansion. J Mater Sci Lett 15:475-477. https://doi.org/10.1007/BF00275406

7. Gu XJ, Zhu JH, Zhang WH (2012) The lattice structure configuration design for stereolithography investment casting pattern using topology optimization. Rapid Prototyp J 18:353-361. https://doi.org/10.1108/13552541211250355

8. Takezawa A, Kobashi M (2017) Design methodology for porous composites with tunable thermal expansion produced by multi- 
material topology optimization and additive manufacturing. Compos Part B $\quad$ Eng 131:21-29. https://doi.org/10.1016/J.COMPOSITESB.2017.07.054

9. Wu T, Liu K, Tovar A (2017) Multiphase topology optimization of lattice injection molds. Comput Struct 192:71-82. https://doi.org/10.1016/J.COMPSTRUC.2017.07.007

10. Bendsøe MP, Sigmund O (2004) Topology optimization by distribution of isotropic material. Springer Berlin Heidelberg, Berlin, Heidelberg

11. Sigmund O, Petersson J (1998) Numerical instabilities in topology optimization: A survey on procedures dealing with checkerboards, mesh-dependencies and local minima. Struct Optim 16:68-75. https://doi.org/10.1007/BF01214002

12. Bendsøe MP, Kikuchi N (1988) Generating optimal topologies in structural design using a homogenization method. Comput Methods Appl Mech Eng 71:197-224. https://doi.org/https://doi.org/10.1016/0045-7825(88)90086-2

13. Guedes J, Kikuchi N (1990) Preprocessing and postprocessing for materials based on the homogenization method with adaptive finite element methods. Comput Methods Appl Mech Eng 83:143-198. https://doi.org/10.1016/0045-7825(90)90148-F

14. Suzuki K, Kikuchi N (1991) A homogenization method for shape and topology optimization. Comput Methods Appl Mech Eng 93:291-318. https://doi.org/10.1016/0045-7825(91)90245-2

15. Allaire G, Jouve F, Maillot $H$ (2004) Topology optimization for minimum stress design with the homogenization method. Struct Multidiscip Optim 28:. https://doi.org/10.1007/s00158-004-0442-8

16. Bendsøe MP (1989) Optimal shape design as a material distribution problem. Struct Optim 1:193-202. https://doi.org/10.1007/BF01650949 
17. Bendsøe MP, Sigmund O (2004) Topology Optimization: Theory, Methods, and Applications. Springer Berlin Heidelberg

18. Huang X, Xie YM (2010) Evolutionary Topology Optimization of Continuum Structures. John Wiley \& Sons, Ltd, Chichester, UK

19. Hörnlein HREM, Kočvara M, Werner R (2001) Material optimization: Bridging the gap between conceptual and preliminary design. Aerosp Sci Technol 5:541-554. https://doi.org/10.1016/S12709638(01)01125-7

20. Chirehdast M, Sankaranarayanan S, Ambo S, Johanson R (1994) Validation of topology optimization for component design. In: 5th Symposium on Multidisciplinary Analysis and Optimization. American Institute of Aeronautics and Astronautics, Reston, Virigina, pp 132137

21. Yang RJ, Chahande Al (1995) Automotive applications of topology optimization. Struct Optim 9:245-249. https://doi.org/10.1007/BF01743977

22. Zhu JH, Zhang WH, Xia L (2016) Topology Optimization in Aircraft and Aerospace Structures Design. Arch Comput Methods Eng 23:595-622. https://doi.org/10.1007/s11831-015-9151-2

23. Fullwood DT, Niezgoda SR, Adams BL, Kalidindi SR (2010) Microstructure sensitive design for performance optimization. Prog. Mater. Sci. 55:477-562

24. Takezawa A, Yonekura $\mathrm{K}$, Koizumi $\mathrm{Y}$, et al (2018) Isotropic Ti-6Al$4 \mathrm{~V}$ lattice via topology optimization and electron-beam melting. Addit Manuf 22:634-642. https://doi.org/10.1016/J.ADDMA.2018.06.008

25. Xu J, Gao L, Xiao M, et al (2020) Isogeometric topology optimization for rational design of ultra-lightweight architected materials. Int J Mech Sci 166:105103. https://doi.org/10.1016/J.IJMECSCI.2019.105103 
26. Ashby MF (1991) Overview No. 92: Materials and shape. Acta Metall Mater 39:1025-1039. https://doi.org/https://doi.org/10.1016/09567151(91)90189-8

27. Bendsøe MP, Díaz A, Kikuchi N (1993) Topology and Generalized Layout Optimization of Elastic Structures BT - Topology Design of Structures. In: Bendsøe MP, Soares CAM (eds). Springer Netherlands, Dordrecht, pp 159-205

28. Xia L, Breitkopf $P$ (2014) Concurrent topology optimization design of material and structure within FE2 nonlinear multiscale analysis framework. Comput Methods Appl Mech Eng 278:524-542. https://doi.org/10.1016/j.cma.2014.05.022

29. Hassani B, Hinton E (1998) A review of homogenization and topology optimization l-homogenization theory for media with periodic structure. Comput Struct 69:707-717. https://doi.org/10.1016/S00457949(98)00131-X

30. Hassani B, Hinton E (1998) A review of homogenization and topology opimization II - Analytical and numerical solution of homogenization equations. Comput 69:719-738. https://doi.org/10.1016/S0045-7949(98)00132-1

31. Michel JC, Moulinec H, Suquet P (1999) Effective properties of composite materials with periodic microstructure: a computational approach. Comput Methods Appl Mech Eng 172:109-143. https://doi.org/10.1016/S0045-7825(98)00227-8

32. Xia L (2016) Multiscale structural topology optimization, 1st ed. ELSEVIER

33. Attaran $M$ (2017) The rise of 3-D printing: The advantages of additive manufacturing over traditional manufacturing. Bus Horiz 60:677-688. https://doi.org/10.1016/j.bushor.2017.05.011

34. Oropallo W, Piegl LA (2016) Ten challenges in 3D printing. Eng 
Comput 32:135-148. https://doi.org/10.1007/s00366-015-0407-0

35. ASTM (2013) F2792 - 12a. Stand Terminol Addit Manuf Technol 10.04: https://doi.org/10.1520/F2792-12A

36. Gaynor AT, Guest JK (2016) Topology optimization considering overhang constraints: Eliminating sacrificial support material in additive manufacturing through design. Struct Multidiscip Optim 54:1157-1172. https://doi.org/10.1007/s00158-016-1551-x

37. Strano G, Hao L, Everson RM, Evans KE (2013) A new approach to the design and optimisation of support structures in additive manufacturing. Int J Adv Manuf Technol 66:1247-1254. https://doi.org/10.1007/s00170-012-4403-x

38. Gaynor AT, Meisel NA, Williams CB, Guest JK (2014) Topology Optimization for Additive Manufacturing: Considering Maximum Overhang Constraint. In: 15th AIAA/ISSMO Multidisciplinary Analysis and Optimization Conference. American Institute of Aeronautics and Astronautics, Reston, Virginia

39. Langelaar M (2016) Topology optimization of 3D self-supporting structures for additive manufacturing. Addit Manuf 12:60-70. https://doi.org/10.1016/j.addma.2016.06.010

40. Jun W, Charlie CLW, Xiaoting Z, Rüdiger W (2016) Self-supporting rhombic infill structures for additive manufacturing. Comput Des 80:32-42. https://doi.org/10.1016/J.CAD.2016.07.006

41. Zhao D, Li M, Liu Y (2017) Self-supporting Topology Optimization for Additive Manufacturing

42. Wu J, Wang CCL, Zhang X, Westermann R (2016) Self-supporting rhombic infill structures for additive manufacturing. Comput Des 80:32-42. https://doi.org/10.1016/J.CAD.2016.07.006

43. Martin L, Luigi M, Federico T, et al (2014) Optimal topology for 
additive manufacture: A method for enabling additive manufacture of support-free optimal structures. Mater Des 63:678-690. https://doi.org/10.1016/J.MATDES.2014.06.015

44. Liu L, Yan J, Cheng G (2008) Optimum structure with homogeneous optimum truss-like material. Comput Struct 86:1417-1425. https://doi.org/10.1016/J.COMPSTRUC.2007.04.030

45. Yan X, Huang X, Zha Y, Xie YM (2014) Concurrent topology optimization of structures and their composite microstructures. Comput Struct 133:103-110. https://doi.org/10.1016/j.compstruc.2013.12.001

46. Querin OM, Steven GP, Xie YM (1998) Evolutionary structural optimisation (ESO) using a bidirectional algorithm. Eng Comput 15:1031-1048. https://doi.org/10.1108/02644409810244129

47. Yang XY, Xei YM, Steven GP, Querin OM (1999) Bidirectional Evolutionary Method for Stiffness Optimization. AIAA J 37:14831488. https://doi.org/10.2514/2.626

48. Wang $Y$, Wang MY, Chen F (2016) Structure-material integrated design by level sets. Struct Multidiscip Optim 54:1145-1156. https://doi.org/10.1007/s00158-016-1430-5

49. Chen W, Tong L, Liu S (2017) Concurrent topology design of structure and material using a two-scale topology optimization. Comput Struct 178:119-128. https://doi.org/10.1016/j.compstruc.2016.10.013

50. Wang $\mathrm{Y}, \mathrm{Xu} \mathrm{H}$, Pasini $\mathrm{D}$ (2017) Multiscale isogeometric topology optimization for lattice materials. Comput Methods Appl Mech Eng 316:568-585. https://doi.org/10.1016/J.CMA.2016.08.015

51. Sivapuram R, Dunning PD, Kim HA (2016) Simultaneous material and structural optimization by multiscale topology optimization. Struct Multidiscip Optim 54:1267-1281. https://doi.org/10.1007/s00158016-1519-x 
52. Li H, Luo Z, Gao L, Qin Q (2018) Topology optimization for concurrent design of structures with multi-patch microstructures by level sets. Comput Methods Appl Mech Eng 331:536-561. https://doi.org/10.1016/J.CMA.2017.11.033

53. Rodrigues H, Guedes JM, Bendsoe MP (2002) Hierarchical optimization of material and structure. Struct Multidiscip Optim 24:110. https://doi.org/10.1007/s00158-002-0209-z

54. Xu M, Xia L, Wang S, et al (2019) An isogeometric approach to topology optimization of spatially graded hierarchical structures. Compos Struct 225:111171. https://doi.org/10.1016/J.COMPSTRUCT.2019.111171

55. Wang Y, Kang Z (2019) Concurrent two-scale topological design of multiple unit cells and structure using combined velocity field level set and density model. Comput Methods Appl Mech Eng 347:340-364. https://doi.org/10.1016/j.cma.2018.12.018

56. Wang MY, Wang X, Guo D (2003) A level set method for structural topology optimization. Comput Methods Appl Mech Eng 192:227246. https://doi.org/10.1016/S0045-7825(02)00559-5

57. Wang $Y$, Kang $Z$ (2018) A velocity field level set method for shape and topology optimization. Int J Numer Methods Eng 115:1315-1336. https://doi.org/10.1002/nme.5845

58. Du Z, Zhou X-Y, Picelli R, Kim HA (2018) Connecting Microstructures for Multiscale Topology Optimization With Connectivity Index $\begin{array}{lllll}\text { Constraints. J Mech } & \text { Des }\end{array}$ https://doi.org/10.1115/1.4041176

59. Schumacher C, Bickel B, Rys J, et al (2015) Microstructures to control elasticity in 3D printing. ACM Trans Graph 34:136:1-136:13. https://doi.org/10.1145/2766926

60. Zhang H, Wang Y, Kang Z (2019) Topology optimization for 
concurrent design of layer-wise graded lattice materials and structures. Int J Eng Sci 138:26-49. https://doi.org/10.1016/J.IJENGSCI.2019.01.006

61. Wang Y, Chen F, Wang MY (2017) Concurrent design with connectable graded microstructures. Comput Methods Appl Mech Eng 317:84-101. https://doi.org/10.1016/J.CMA.2016.12.007

62. Zhou S, Li Q (2008) Design of graded two-phase microstructures for tailored elasticity gradients. J Mater Sci 43:5157-5167. https://doi.org/10.1007/s10853-008-2722-y

63. Radman A, Huang X, Xie YM (2013) Topology optimization of functionally graded cellular materials. J Mater Sci 48:1503-1510. https://doi.org/10.1007/s10853-012-6905-1

64. Garner E, Kolken HMA, Wang CCL, et al (2019) Compatibility in microstructural optimization for additive manufacturing. Addit Manuf 26:65-75. https://doi.org/10.1016/J.ADDMA.2018.12.007

65. Sigmund O (2020) EML webinar overview: Topology Optimization Status and Perspectives. Extrem Mech Lett 39:100855. https://doi.org/10.1016/j.eml.2020.100855

66. Zeinalabedini H, Yildiz YO, Zhang P, et al (2016) Homogenization of additive manufactured polymeric foams with spherical cells. Addit Manuf 12:274-281. https://doi.org/10.1016/j.addma.2016.04.008

67. Lee T, Lee J, Lee K (2017) Extended block based infill generation. Int J Adv Manuf Technol 93:1415-1430. https://doi.org/10.1007/s00170017-0572-y

68. Lee J, Lee K (2017) Block-based inner support structure generation algorithm for 3D printing using fused deposition modeling. Int $\mathrm{J}$ Adv Manuf Technol 89:2151-2163. https://doi.org/10.1007/s00170-0169239-3 
69. Lu L, Sharf A, Zhao H, et al (2014) Build-to-last: Strength to weight 3D printed objects. In: ACM Transactions on Graphics. Association for Computing Machinery, pp 1-10

70. Panesar A, Abdi M, Hickman D, Ashcroft I (2018) Strategies for functionally graded lattice structures derived using topology optimisation for Additive Manufacturing. Addit Manuf 19:81-94. https://doi.org/10.1016/J.ADDMA.2017.11.008

71. Robbins J, Owen SJ, Clark BW, Voth TE (2016) An efficient and scalable approach for generating topologically optimized cellular structures for additive manufacturing. Addit Manuf 12:296-304. https://doi.org/10.1016/j.addma.2016.06.013

72. Ashby MF (2006) The properties of foams and lattices. Philos Trans A Math Phys Eng Sci 364:15-30. https://doi.org/10.1098/rsta.2005.1678

73. Fuller (1961) Octet Truss

74. Deshpande VS, Fleck NA, Ashby MF (2001) Effective properties of the octet-truss lattice material. J Mech Phys Solids 49:1747-1769. https://doi.org/10.1016/S0022-5096(01)00010-2

75. Du Y, Li H, Luo Z, Tian Q (2017) Topological design optimization of lattice structures to maximize shear stiffness. Adv Eng Softw 112:211-221. https://doi.org/10.1016/J.ADVENGSOFT.2017.04.011

76. Cheng L, Liang X, Belski E, et al (2018) Natural Frequency Optimization of Variable-Density Additive Manufactured Lattice Structure: Theory and Experimental Validation. J Manuf Sci Eng 140:. https://doi.org/10.1115/1.4040622

77. Wang X, Zhang P, Ludwick S, et al (2018) Natural frequency optimization of 3D printed variable-density honeycomb structure via a homogenization-based approach. Addit Manuf 20:189-198. https://doi.org/10.1016/J.ADDMA.2017.10.001 
78. Primo T, Calabrese M, Del Prete A, Anglani A (2017) Additive manufacturing integration with topology optimization methodology for innovative product design. Int $\mathrm{J}$ Adv Manuf Technol 93:467-479. https://doi.org/10.1007/s00170-017-0112-9

79. Han Y, Lu WF (2018) A Novel Design Method for Nonuniform Lattice Structures Based on Topology Optimization. J Mech Des 140: https://doi.org/10.1115/1.4040546

80. Wu J, Aage N, Westermann R, Sigmund O (2018) Infill Optimization for Additive Manufacturing-Approaching Bone-Like Porous Structures. IEEE Trans Vis Comput Graph 24:1127-1140. https://doi.org/10.1109/TVCG.2017.2655523

81. Liu J, Gaynor AT, Chen S, et al (2018) Current and future trends in topology optimization for additive manufacturing. Struct Multidiscip Optim 57:2457-2483. https://doi.org/10.1007/s00158-018-1994-3

82. Niu B, Yan J, Cheng G (2009) Optimum structure with homogeneous optimum cellular material for maximum fundamental frequency. Struct Multidiscip Optim 39:115-132. https://doi.org/10.1007/s00158-0080334-4

83. Coelho PG, Fernandes PR, Guedes JM, Rodrigues HC (2008) A hierarchical model for concurrent material and topology optimisation of three-dimensional structures. Struct Multidiscip Optim 35:107-115. https://doi.org/10.1007/s00158-007-0141-3

84. Gao J, Luo Z, Xia L, Gao L (2019) Concurrent topology optimization of multiscale composite structures in Matlab. Struct Multidiscip Optim 60:2621-2651. https://doi.org/10.1007/s00158-019-02323-6

85. Yan J, Guo X, Cheng G (2016) Multi-scale concurrent material and structural design under mechanical and thermal loads. Comput Mech 57:437-446. https://doi.org/10.1007/s00466-015-1255-x

86. Deng J, Yan J, Cheng G (2013) Multi-objective concurrent topology 
optimization of thermoelastic structures composed of homogeneous porous material. Struct Multidiscip Optim 47:583-597. https://doi.org/10.1007/s00158-012-0849-6

87. Cheng G, Xu L (2016) Two-scale topology design optimization of stiffened or porous plate subject to out-of-plane buckling constraint. Struct Multidiscip Optim 54:1283-1296. https://doi.org/10.1007/s00158-016-1542-y

88. Hughes TJR, Cottrell JA, Bazilevs $Y(2005)$ Isogeometric analysis: CAD, finite elements, NURBS, exact geometry and mesh refinement. Comput Methods Appl Mech Eng 194:4135-4195. https://doi.org/10.1016/J.CMA.2004.10.008

89. Gao J, Luo Z, Li H, Gao L (2019) Topology optimization for multiscale design of porous composites with multi-domain microstructures. Comput Methods Appl Mech Eng 344:451-476. https://doi.org/10.1016/J.CMA.2018.10.017

90. Wang $\mathrm{C}$, Zhu JH, Zhang $\mathrm{WH}$, et al (2018) Concurrent topology optimization design of structures and non-uniform parameterized lattice microstructures. Struct Multidiscip Optim 58:35-50. https://doi.org/10.1007/s00158-018-2009-0

91. Deng J, Chen W (2017) Concurrent topology optimization of multiscale structures with multiple porous materials under random field loading uncertainty. Struct Multidiscip Optim 56:1-19. https://doi.org/10.1007/s00158-017-1689-1

92. Turk G, O'Brien JF (1999) Shape transformation using variational implicit functions. In: Proceedings of the 26th Annual Conference on Computer Graphics and Interactive Techniques, SIGGRAPH 1999. Association for Computing Machinery, Inc, New York, New York, USA, pp 335-342

93. Niino M, Maeda S (1990) Recent Development Status of Functionally Gradient Materials. ISIJ Int 30:699-703. 
https://doi.org/10.2355/isijinternational.30.699

94. Kalpakjian S, Schmid SR, Sánchez García G (2008) Manufactura, ingeniería y tecnología, 5th ed. Pearson Educación, Mexico

95. Liou FW (2008) Rapid prototyping and engineering applications : a toolbox for prototype development. CRC Press

96. Bertsch A, Renaud P (2020) Microstereolithography. In: ThreeDimensional Microfabrication Using Two-Photon Polymerization. Elsevier, pp 25-56

97. Suzuki $\mathrm{Y}$, Tahara $\mathrm{H}$, Michihata $\mathrm{M}$, et al (2016) Evanescent Light Exposing System under Nitrogen Purge for Nano-Stereolithography. Procedia CIRP 42:77-80. https://doi.org/10.1016/j.procir.2016.02.192

98. Takahashi S, Kajihara Y, Takamasu K (2012) Submicrometer thickness layer fabrication for layer-by-layer microstereolithography using evanescent light. CIRP Ann 61:219-222. https://doi.org/10.1016/j.cirp.2012.03.069

99. Zhou J, Lu Z, Miao K, et al (2015) Quick fabrication of aeronautical complicated structural parts based on stereolithography. Propuls Power Res 4:63-71. https://doi.org/10.1016/j.jppr.2015.05.001

100. 3D systems Lotus F1 Team and 3D Systems move together towards race-ready mass production of parts | 3D Systems. http://www.3dsystems.com/learning-center/case-studies/lotus-f1team-and-3d-systems-move-together-towards-race-ready-mass. Accessed 4 Jan 2017

101. Anderl H, Zur Nedden D, Mu"hlbauer W, et al (1994) CT-guided stereolithography as a new tool in craniofacial surgery. Br. J. Plast. Surg. 47:60-64

102. Álvarez Quesada C, Quesada Á, Baracaldo C, et al (2006) Avances 
en equipamientos (I): la Estereolitografía y sus materiales, un paso hacia el futuro. 151-156

103. Bill JS, Reuther JF, Dittmann W, et al (1995) Stereolithography in oral and maxillofacial operation planning. Int J Oral Maxillofac Surg 24:98103. https://doi.org/10.1016/S0901-5027(05)80869-0

104. Arvier JF, Barker TM, Yau YY, et al (1994) Maxillofacial biomodelling. $\mathrm{Br} J$ Oral Maxillofac Surg 32:276-283. https://doi.org/10.1016/02664356(94)90046-9

105. Kawata S, Sun HB, Tanaka T, Takada K (2001) Finer features for functional microdevices. Nature 412:697-698. https://doi.org/10.1038/35089130

106. Lü L, Fuh JYH, Wong YS (2001) Laser-Induced Materials and Processes for Rapid Prototyping. Springer US, Boston, MA

107. Proto Labs Internacional Direct Metal Laser Sintering DMLS. https://www.protolabs.com/3d-printing/direct-metal-laser-sintering/. Accessed 7 Jan 2017

108. Kathuria YP (1999) Microstructuring by selective laser sintering of metallic powder. Surf Coatings Technol 116-119:643-647. https://doi.org/10.1016/S0257-8972(99)00266-2

109. Kathuria YP (1997) Selective laser sintering of metallic powder for microfabrication technology. In: 1997 International Symposium on Micromechanics and Human Science (Cat. No.97TH8311). IEEE, pp $41-47$

110. Singer neal. (1997) Intense hopes: Ten companies team to commercialize Sandia's powder-to-parts net shaping technology Computer-controlled lasers can create complex custom parts or molds from metallic powder in a day. In: Sandia, LabNews. http://www.sandia.gov/LabNews/LN12-05-97/lens_story.html. Accessed 7 Jan 2017 
111. 3D Systems (2014) 3D Systems and Ekso Bionics Help Man and Machine Walk as One | 3D Systems. https://www.3dsystems.com/learning-center/case-studies/3dsystems-and-ekso-bionics-help-man-and-machine-walk-one. Accessed 7 Jan 2017

112. Cooper KG (2001) Rapid prototyping technology: selection and application. Marcel Dekker, New York, NY

113. Masood S., Song W. (2004) Development of new metal/polymer materials for rapid tooling using Fused deposition modelling. Mater Des 25:587-594. https://doi.org/10.1016/j.matdes.2004.02.009

114. Novakova-Marcincinova L, Novak-Marcincin J, Barna J, Torok J (2012) Special materials used in FDM rapid prototyping technology application. In: 2012 IEEE 16th International Conference on Intelligent Engineering Systems (INES). IEEE, pp 73-76

115. González-Estrada OA, Pertuz Comas AD, Díaz Rodríguez JG (2020) Monotonic load datasets for additively manufactured thermoplastic reinforced composites. Data $\mathrm{Br}$ 29:105295. https://doi.org/10.1016/j.dib.2020.105295

116. Kroll E, Artzi D (2011) Enhancing aerospace engineering students' learning with $3 \mathrm{D}$ printing wind-tunnel models. Rapid Prototyp J 17:393-402. https://doi.org/10.1108/13552541111156522

117. Artero Guerrero JA, Pernas-Sánchez J, Varas D, et al (2016) Análisis experimental de estructura aeroespaciales fabricadas mediante impresión 3D. Universidad Carlos III de Madrid

118. Bixby Mera S (2014) Feasibility study for the manufacturing of a shock absorber using 3D printing technology. Sydney / Universidad de Sydney / 2014

119. Sandler N, Salmela I, Fallarero A, et al (2014) Towards fabrication of 3D printed medical devices to prevent biofilm formation. Int J Pharm 
459:62-64. https://doi.org/10.1016/j.ijpharm.2013.11.001

120. Yamazaki T (2016) Development of A Hybrid Multi-tasking Machine Tool: Integration of Additive Manufacturing Technology with CNC Machining. Procedia CIRP 42:81-86. https://doi.org/10.1016/j.procir.2016.02.193

121. Rankin TM, Giovinco NA, Cucher DJ, et al (2014) Three-dimensional printing surgical instruments: are we there yet? J Surg Res 189:193197. https://doi.org/10.1016/j.jss.2014.02.020

122. Stratasys Functional Medical Tool Prototypes Improve Design. http://www.stratasys.com/resources/case-studies/medical/medtronic. Accessed 11 Jan 2017

123. Cardiac Design Labs Medical Case Study: Cardiac Design Labs Bangalore-Based Medical Start-Up Builds Next-Generation Medical System with FDM Technologyl Stratasys. http://www.stratasys.com/resources/case-studies/medical/cardiacdesign-labs. Accessed 11 Jan 2017

124. Wong JY, Pfahnl AC (2014) 3D Printing of Surgical Instruments for Long-Duration Space Missions. Aviat Space Environ Med 85:758763. https://doi.org/10.3357/ASEM.3898.2014

125. Gibson I, Kvan T, Wai Ming L (2002) Rapid prototyping for architectural models. Rapid Prototyp J 8:91-95. https://doi.org/10.1108/13552540210420961

126. Ryder G, Ion B, Green G, et al (2002) Rapid design and manufacture tools in architecture. Autom Constr 11:279-290. https://doi.org/10.1016/S0926-5805(00)00111-4

127. Stokes MB (2013) 3D Printing for Architects with MakerBot, 1st ed. Packt Publishing

128. Sher D, Tutó X (2015) La impressió d'aliments en 3D. Temes de 
disseny 0:104-117

129. Wei-Hsiang Lai C-IC (2007) Manufacturing method of threedimensional food by rapid prototyping

130. Sun J, Peng Z, Yan L, et al (2015) 3D food printing-An innovative way of mass customization in food fabrication. Int $\mathrm{J}$ Bioprinting 1 :. https://doi.org/10.18063/lJB.2015.01.006

131. Natural Machines Natural Machines: The makers of Foodini - a 3D food printer making all types of fresh, nutritious foods. https://www.naturalmachines.com/. Accessed 12 Jan 2017

132. Rengier F, Mehndiratta A, von Tengg-Kobligk H, et al (2010) 3D printing based on imaging data: review of medical applications. Int $\mathrm{J}$ Comput Assist Radiol Surg 5:335-341. https://doi.org/10.1007/s11548-010-0476-x

133. McGurk M, Amis AA, Potamianos P, Goodger NM (1997) Rapid prototyping techniques for anatomical modelling in medicine. Ann $R$ Coll Surg Engl 79:169-74

134. Hutmacher DW (2000) Scaffolds in tissue engineering bone and cartilage. Biomaterials $21: 2529-2543$. https://doi.org/10.1016/S01429612(00)00121-6

135. Skowyra J, Pietrzak K, Alhnan MA (2015) Fabrication of extendedrelease patient-tailored prednisolone tablets via fused deposition modelling (FDM) 3D printing. Eur $J$ Pharm Sci 68:11-17. https://doi.org/10.1016/j.ejps.2014.11.009

136. Martin Chung-ming W Medical Case Study: Hong Kong Polytechnic University Hong Kong Polytechnic University uses 3D printing to improve oye orbital implant surgery. http://www.stratasys.com/resources/case-studies/medical/hongkong-polytechnic-university. Accessed 11 Jan 2017 
137. Calì J, Calian DA, Amati C, et al (2012) 3D-printing of non-assembly, articulated models. ACM Trans Graph 31:1. https://doi.org/10.1145/2366145.2366149

138. AnnaC A Una línea de juguetes, con distintas discapacidades, creada con una impresora 3D | TECNONAUTA. http://www.tecnonauta.com/noticias/2423-juguetes-creados-conimpresora-3d-con-discapacidad. Accessed 12 Jan 2017

139. Prager W, Taylor JE (1968) Problems of Optimal Structural Design. J Appl Mech 35:102-106. https://doi.org/10.1115/1.3601120

140. Olhoff N (1970) Optimal design of vibrating circular plates. Int J Solids Struct 6:139-156. https://doi.org/https://doi.org/10.1016/00207683(70)90086-7

141. Taylor JE, Rossow MP (1977) Optimal truss design based on an algorithm using optimality criteria. Int J Solids Struct 13:913-923. https://doi.org/https://doi.org/10.1016/0020-7683(77)90004-X

142. Bendsøe MP (1986) Generalized Plate Models and Optimal Design BT - Homogenization and Effective Moduli of Materials and Media. In: Ericksen JL, Kinderlehrer D, Kohn R, Lions J-L (eds). Springer New York, New York, NY, pp 1-26

143. Andreassen $E$, Clausen $A$, Schevenels $M$, et al (2011) Efficient topology optimization in MATLAB using 88 lines of code. Struct Multidiscip Optim 43:1-16. https://doi.org/10.1007/s00158-010-05947

144. Sigmund O (2001) A 99 line topology optimization code written in Matlab. Struct Multidiscip Optim 21:120-127. https://doi.org/10.1007/s001580050176

145. Díaz A, Sigmund O (1995) Checkerboard patterns in layout optimization. Struct Optim 10:40-45. https://doi.org/10.1007/BF01743693 
146. Bourdin B (2001) Filters in topology optimization. Int J Numer Methods Eng 50:2143-2158. https://doi.org/10.1002/nme.116

147. Bendsøe MP, Sigmund O (1999) Material interpolation schemes in topology optimization. Arch Appl Mech (Ingenieur Arch 69:635-654. https://doi.org/10.1007/s004190050248

148. Zhou M, Rozvany GIN (1991) The COC algorithm, Part II: Topological, geometrical and generalized shape optimization. Comput Methods Appl Mech Eng 89:309-336. https://doi.org/10.1016/0045-7825(91)90046-9

149. Mlejnek HP, Schirrmacher R (1993) An engineer's approach to optimal material distribution and shape finding. Comput Methods Appl Mech Eng 106:1-26. https://doi.org/https://doi.org/10.1016/00457825(93)90182-W

150. Sigmund $O$ (2007) Morphology-based black and white filters for topology optimization. Struct Multidiscip Optim 33:401-424. https://doi.org/10.1007/s00158-006-0087-x

151. Allaire G, Jouve F, Toader A-M (2004) Structural optimization using sensitivity analysis and a level-set method. J Comput Phys 194:363393. https://doi.org/https://doi.org/10.1016/j.jcp.2003.09.032

152. Stegmann J, Lund E (2005) Discrete material optimization of general composite shell structures. Int J Numer Methods Eng 62:2009-2027. https://doi.org/10.1002/nme.1259

153. Hvejsel CF, Lund E (2011) Material interpolation schemes for unified topology and multi-material optimization. Struct Multidiscip Optim 43:811-825. https://doi.org/10.1007/s00158-011-0625-z

154. Hvejsel CF, Lund E, Stolpe M (2011) Optimization strategies for discrete multi-material stiffness optimization. Struct Multidiscip Optim 44:149-163. https://doi.org/10.1007/s00158-011-0648-5 
155. Phani AS, Hussein MI (2017) Dynamics of lattice materials, 1st ed. John Wiley \& Sons, Ltd, chichester

156. Nelli Silva EC, Nishiwaki S, Fonseca JSO, et al (1998) Topology optimization applied to the design of piezocomposite materials and piezoelectric actuators. In: Varadan V V. (ed). International Society for Optics and Photonics, pp 216-227

157. Vatanabe SL, Paulino GH, Silva ECN (2013) Design of functionally graded piezocomposites using topology optimization and homogenization - Toward effective energy harvesting materials. Comput Methods Appl Mech Eng 266:205-218. https://doi.org/10.1016/J.CMA.2013.07.003

158. Carbonari RC, Silva ECN, Paulino GH (2009) Multi-actuated functionally graded piezoelectric micro-tools design: A multiphysics topology optimization approach. Int J Numer Methods Eng 77:301336. https://doi.org/10.1002/nme.2403

159. Carbonari RC, Silva ECN, Paulino GH (2007) Topology optimization design of functionally graded bimorph-type piezoelectric actuators. Smart Mater Struct 16:2605-2620. https://doi.org/10.1088/0964$1726 / 16 / 6 / 065$

160. Nelli Silva EC, Ono Fonseca JS, de Espinosa FM, et al (1999) Design of piezocomposite materials and piezoelectric transducers using topology optimization-Part I. Arch Comput Methods Eng 6:117-182. https://doi.org/10.1007/BF02736183

161. Nelli Silva EC, Nishiwaki S, Ono Fonseca JS, Kikuchi N (1999) Optimization methods applied to material and flextensional actuator design using the homogenization method. Comput Methods Appl Mech Eng 172:241-271. https://doi.org/10.1016/S00457825(98)00231-X

162. Cardoso, Eduardo. Sergio J, Fonseca O (2004) Strain energy maximization approach to the design of fully compliant mechanisms 
using topology optimization. Lat Am J Solids Struct

163. Rubio WM, Paulino GH, Silva ECN (2011) Tailoring vibration mode shapes using topology optimization and functionally graded material concepts. Smart Mater Struct 20:025009. https://doi.org/10.1088/0964-1726/20/2/025009

164. Carniel TA, Fancello EA (2019) A variational homogenization approach applied to the multiscale analysis of the viscoelastic behavior of tendon fascicles. Contin Mech Thermodyn 31:607-626. https://doi.org/10.1007/s00161-018-0714-y

165. Carniel TA, Klahr B, Fancello EA (2019) On multiscale boundary conditions in the computational homogenization of an RVE of tendon fascicles. J Mech Behav Biomed Mater 91:131-138. https://doi.org/10.1016/J.JMBBM.2018.12.003

166. Salmoria G V., Fancello EA, Roesler CRM, Dabbas F (2013) Functional graded scaffold of HDPE/HA prepared by selective laser sintering: microstructure and mechanical properties. Int J Adv Manuf Technol 65:1529-1534. https://doi.org/10.1007/s00170-012-4277-y

167. Ostoja-Starzewski M (2002) Microstructural randomness versus representative volume element in thermomechanics. J Appl Mech Trans ASME 69:25-35. https://doi.org/10.1115/1.1410366

168. Taghite M, Rahmattulla A, Lanchon-Ducauquis H, Taous K (1997) Homogenization of a thermal problem in the plate of a heat exchanger. Comput Methods Appl Mech Eng 145:381-402. https://doi.org/10.1016/S0045-7825(96)01214-5

169. Laschet G (2002) Homogenization of the thermal properties of transpiration cooled multi-layer plates. Comput Methods Appl Mech Eng 191:4535-4554. https://doi.org/10.1016/S0045-7825(02)003195

170. Carniel TA, Klahr B, Fancello EA (2018) A multiscale numerical 
approach for the finite strains analysis of materials reinforced with helical fibers. Mech Mater 126:75-85. https://doi.org/10.1016/J.MECHMAT.2018.07.014

171. Hashin Z (1983) Analysis of Composite Materials-A Survey. J Appl Mech 50:481. https://doi.org/10.1115/1.3167081

172. Xia L, Breitkopf $P$ (2015) Design of materials using topology optimization and energy-based homogenization approach in Matlab. Struct Multidiscip Optim 52:1229-1241. https://doi.org/10.1007/s00158-015-1294-0

173. Sigmund O (1994) Materials with prescribed constitutive parameters: An inverse homogenization problem. Int J Solids Struct 31:23132329. https://doi.org/10.1016/0020-7683(94)90154-6

174. Bendsoe MP, Sigmund O (2003) Topology Optimization: Theory, Methods, and Applications. Springer Berlin Heidelberg

175. Xia Z, Zhang Y, Ellyin F (2003) A unified periodical boundary conditions for representative volume elements of composites and applications. Int J Solids Struct 40:1907-1921. https://doi.org/10.1016/S0020-7683(03)00024-6

176. AAAS., Advancing Science Serving Society The Cells in Your Body Science NetLinks. http://sciencenetlinks.com/student-teachersheets/cells-your-body/. Accessed 20 Dec 2019

177. Lieber RL, Lieber RL (2002) Skeletal muscle structure, function \&amp; plasticity: the physiological basis of rehabilitation. Lippincott Williams \& Wilkins

178. ASEM, AFM (2003) El Músculo esquelético. Vigo, España.

179. Olhoff N, Bendsøe MP, Rasmussen J (1991) On CAD-integrated structural topology and design optimization. Comput Methods Appl Mech Eng 89:259-279. https://doi.org/10.1016/0045-7825(91)90044- 
7

180. The Mathworks Inc (2019) Matlab 2019b

181. Payten WM, Ben-Nissan B, Mercert DJ (1998) Optimal topology design using a global self-organisational approach. Int J Solids Struct 35:219-237.

https://doi.org/https://doi.org/10.1016/S00207683(97)00064-4

182. Igarashi $\mathrm{H}$, Honma $\mathrm{T}$ (1996) A boundary element method for potential fields with corner singularities. Appl Math Model 20:847-852. https://doi.org/https://doi.org/10.1016/S0307-904X(96)00091-1

183. Engwirda D (2014) Locally optimal Delaunay-refinement and optimisation-based mesh generation. University of Sydney Faculty of Science School of Mathematics and Statistics

184. EOS FORMIGA P 110 - laser sintering 3D printer - EOS. https://www.eos.info/systems_solutions/plastic/systems_equipment/f ormiga_p_110. Accessed 4 Jan 2020

185. Li H, Luo Z, Gao L, Walker P (2018) Topology optimization for functionally graded cellular composites with metamaterials by level sets. Comput Methods Appl Mech Eng 328:340-364. https://doi.org/10.1016/j.cma.2017.09.008

186. Yan X, Huang X, Sun G, Xie YM (2015) Two-scale optimal design of structures with thermal insulation materials. Compos Struct 120:358365. https://doi.org/10.1016/j.compstruct.2014.10.013

187. (2016) Exploiting Additive Manufacturing Infill in Topology Optimization for Improved Buckling Load. Engineering 2:250-257. https://doi.org/10.1016/J.ENG.2016.02.006

188. Meneses G, Pereira A, Menezes I (2018) Lattice Structures Design by Means of Topology Optimization. Mecánica Comput 36:21112120 
189. Ultimaker Cura: Powerful, easy-to-use 3D printing software. https:/ultimaker.com/software/ultimaker-cura. Accessed 20 Jan 2021

190. Jia J, Da D, Loh C-L, et al (2020) Multiscale topology optimization for non-uniform microstructures with hybrid cellular automata. Struct Multidiscip Optim 62:757-770. https://doi.org/10.1007/s00158-02002533-3

191. Talischi C, Paulino GH, Pereira A, Menezes IFM (2012) PolyMesher: A general-purpose mesh generator for polygonal elements written in Matlab. Struct Multidiscip Optim 45:309-328. https://doi.org/10.1007/s00158-011-0706-z

192. Talischi C, Paulino GH, Pereira A, Menezes IFM (2012) PolyTop: a Matlab implementation of a general topology optimization framework using unstructured polygonal finite element meshes. Struct Multidiscip Optim 45:329-357. https://doi.org/10.1007/s00158-0110696-x 


\section{Appendix}

\section{Variable-density}

In our study, the well-known MBB-beam problem will be used to illustrate the Variable-density method. The design domain and boundary conditions are provided in Figure A-1 a). Using PolyMesher [191] was constructed a $270 \times 90$ uniform quadrilateral mesh and PolyTop [192] was employed to minimize the compliance of the structure. The volume fraction is prescribed as volfrac $=42 \%$ of the initial volume, the applied load is $\mathrm{F}=1000 \mathrm{~N}$, Young's modulus is $\mathrm{E}=210 \mathrm{GPa}$, the Poisson's ratio is $\mathrm{v}=0.3$, and the radius of the filter is $\mathrm{R}=0.06$.

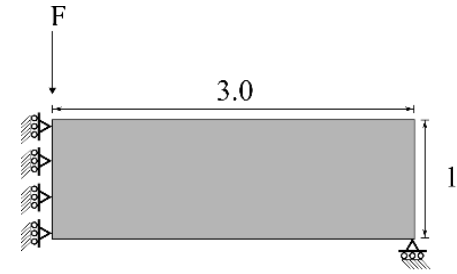

a)

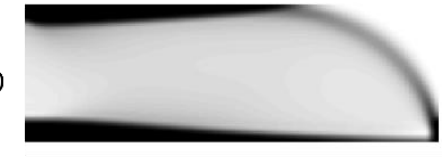

b)

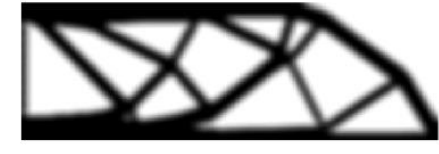

c)

Figure A-1 - Topology optimization. a) Half MBB-beam b) $p=1$ b) $p=1-3$.

Figure A-1 shows the final topologies using the SIMP approach with a fixed penalization factor $\mathrm{p}=1$ (a) and with an increasing penalization factor from $\mathrm{p}=$ 1-3, with increments of 1 (b). Using the final topology shown (Figure A-1 b), the lattice solution (graded lattice) is obtained by replacing a group of $9 \times 9$ elements by a unit cell with the average of the volume fraction values, giving a total of $60 \times$ 10 cells. The result for the MBB-beam Variable-density is shown in Figure A-2.

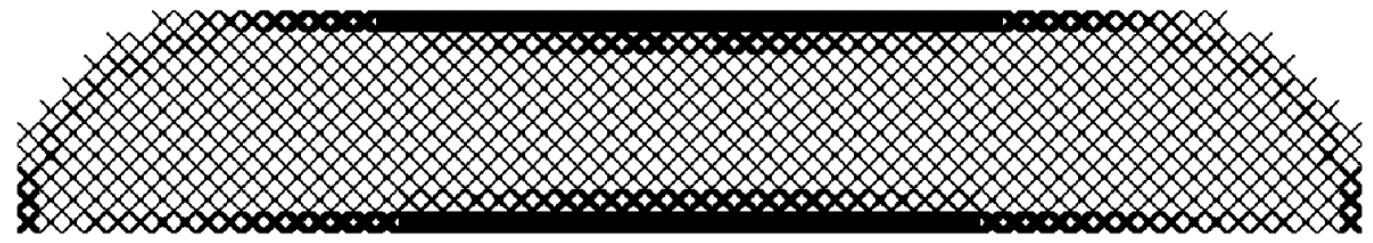

Figure A-2 - MBB-beam Variable-density. 